\title{
Nonlinear Mechanics of Surface Growth for Cylindrical and Spherical Elastic Bodies*
}

\author{
Fabio Sozio ${ }^{1,2}$ and Arash Yavari ${ }^{\dagger 2,3}$ \\ ${ }^{1}$ Department of Mechanical Engineering, Sapienza University of Rome, Rome, Italy \\ ${ }^{2}$ School of Civil and Environmental Engineering, Georgia Institute of Technology, Atlanta, GA 30332, USA \\ ${ }^{3}$ The George W. Woodruff School of Mechanical Engineering, Georgia Institute of Technology, Atlanta, GA 30332, USA
}

August 28, 2016

\begin{abstract}
In this paper we formulate the initial-boundary value problems of accreting cylindrical and spherical nonlinear elastic solids in a geometric framework. It is assumed that the body grows as a result of addition of new (stress-free or pre-stressed) material on part of its boundary. We construct Riemannian material manifolds for a growing body with metrics explicitly depending on the history of applied external loads and deformation during accretion and the growth velocity. We numerically solve the governing equilibrium equations in the case of neo-Hookean solids and compare the accretion and residual stresses with those calculated using the linear mechanics of surface growth.
\end{abstract}

Keywords: Surface growth; accretion mechanics; geometric mechanics; nonlinear elasticity, residual stress.

\section{Contents}

1 Introduction 2

2 Geometric Anelasticity and the Mechanics of Growth 4

3 Attachment of a Stress-Free Layer to a Deformed Infinitely Long Thick Hollow Cylinder 7

3.1 Construction of a material manifold . . . . . . . . . . . . . . . . . . . . . . . . 8

3.2 Calculation of residual stresses for an incompressible isotropic solid . . . . . . . . . . . . . 11

4 Continuous Surface Growth of an Infinitely Long Thick Hollow Cylinder 12

4.1 Outer Accretion . . . . . . . . . . . . . . . . . . . . . . . . . 12

4.1.1 Kinematics and material metric . . . . . . . . . . . . . . . . . . . . . . . . . . . . . . . . . .

4.1.2 Calculation of stresses for an incompressible isotropic accreting cylinder . . . . . . . . . 15

4.1.3 Calculation of residual stresses for an incompressible isotropic accreting cylinder . . . . . 18

4.1.4 Accretion stresses in the linearized theory . . . . . . . . . . . . . . . . . . 19

4.2 Inner Accretion . . . . . . . . . . . . . . . . . . . . . . . . . . 23

4.2.1 Kinematics and material metric . . . . . . . . . . . . . . . . . . . . . 24

4.2.2 Calculation of stresses for an incompressible isotropic accreting cylinder . . . . . . . . . 25

4.2.3 Calculation of residual stresses for an incompressible isotropic accreting cylinder . . . . . 26

4.2.4 Accretion stresses in the linearized theory . . . . . . . . . . . . . . . . 26

*Dedicated to the memory of Professor William S. Klug (1976-2016).

${ }^{\dagger}$ Corresponding author, e-mail: arash.yavari@ce.gatech.edu 
5 Surface Growth of a Thick Hollow Solid Sphere $\quad 28$

5.1 Kinematics and material metric . . . . . . . . . . . . . . . . . . . . . 29

5.2 Calculation of stresses for an incompressible isotropic accreting spherical ball . . . . . . . . 30

5.3 Calculation of residual stresses for an incompressible isotropic accreting spherical ball . . . . . 31

5.4 Surface growth stresses in the linearized theory . . . . . . . . . . . . . . . . . . 32

6 Numerical Examples $\quad 34$

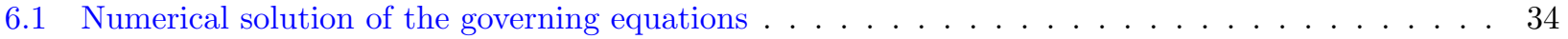

6.2 Numerical results . . . . . . . . . . . . . . . . . . . . . . . . . 35

7 Conclusions

\section{Introduction}

Mechanics of growing bodies has attracted many researchers in recent years partly because of the emerging applications in biomechanics. Nonetheless, structures that are built by a process of accretion are common in engineering, e.g. built up of a concrete dam in successive layers, metal solidification [Schwerdtfeger et al., 1998], electrolytic deposition, layer-by-layer gluing of composites, wound rolls [Altmann, 1968; Yagoda, 1980], thermal and laser-based 3D printing, etc. As examples of surface growth in Nature one can mention volcanic and sedimentary rock formation, ice and snow cover build-up [Brown et al., 1972], formation of planetary objects [Brown and Goodman, 1963], equilibrium shape configurations for rubble piles, the growth of biological tissues [Skalak et al., 1997], etc.

In a growing body mass is no longer conserved and the body may be residually stressed. There are two types of growth: bulk growth and surface growth (or accretion). In bulk growth, in the language of continuum mechanics, material points are preserved; only the mass density and the natural (stress-free) configuration of the body evolve. Because of its similarity with finite plasticity the idea of the decomposition of the deformation gradient into elastic and growth parts was borrowed from plasticity (see [Sadik and Yavari, 2016a] for a historical perspective). There are many theoretical and computational works in the literature of (bulk) growth mechanics, see [Takamizawa and Matsuda, 1990; Takamizawa, 1991; Rodriguez et al., 1994; Epstein and Maugin, 2000; Garikipati et al., 2004; Ben Amar and Goriely, 2005; Klarbring et al., 2007; Yavari, 2010; Sadik et al., 2016] and references therein. In surface growth, instead, new material points are added to or removed from the boundary of the body, meaning that the set of material points evolves in time. Moreover, the relaxed (natural) configuration of the body explicitly depends not only on the surface growth characteristics (accretion flux, accretion velocity, etc.), but also on the history of loading and deformation during surface growth. In the geometric language the material manifold is time dependent and has a metric that depends on both the surface growth characteristics and the loading and deformation during the accretion process. We should emphasize that the mechanics of surface growth is much less developed compared to that of bulk growth perhaps because of its complexity.

The theoretical studies of the mechanics of surface growth go back to the 1940s and the work of Southwell [1941] who analyzed thick-walled cylinders manufactured by wire winding of an initial elastic tube (see [Naumov, 1994] for a more detailed historical accounts of the mechanics of surface growth). Brown and Goodman [1963] studied the problem of a growing planet subject to self-gravity. The analysis was carried out in the framework of the linear theory of elasticity and the growing planet was treated as a hollow sphere made of an isotropic elastic and compressible material. Their approach consisted in considering an incremental elastic problem at each stage of surface growth in which the contribution of self-gravity was taken into account by replacing the body force with a traction boundary condition representing the infinitesimal weight of the added layer. The authors assumed that the infinitesimal contribution of stress, which can also be seen as the time rate of the Cauchy stress $\dot{\boldsymbol{\sigma}},{ }^{1}$ is compatible, and then they integrated it to obtain the total Cauchy stress as:

$$
\boldsymbol{\sigma}(x, t)=\int_{\tau(x)}^{t} \dot{\boldsymbol{\sigma}}(x, s) d s+\boldsymbol{\sigma}(x, \tau(x)),
$$

\footnotetext{
${ }^{1}$ Note that here $\boldsymbol{\sigma}$ is the rate of the Cauchy stress in an accretion process. Surface growth does not need to be parametrized by time. However, if it is parametrized by time, then $\dot{\boldsymbol{\sigma}}$ is a time rate. One needs to be careful with time rates in nonlinear elasticity as not every time rate is objective (frame-indifferent). Such issues do not arise in linear elasticity. In particular, linearizing any of the objective rates of the Cauchy stress with respect to a stress-free configuration one simply obtains the partial derivative of the Cauchy stress with respect to time.
} 
where $\tau(x)$ is the time of addition of the particle occupying the position $x$ (since they work in the setting of linear elasticity it is possible to identify each particle with its spatial position). The second term is the stress tensor on the accretion surface at the point $x$ at its time of addition $\tau(x)$. In the case of an accreting planet resulting from the addition of stress-free material, this term vanishes. They observed that stresses induced by surface growth do not satisfy the Beltrami-Michell compatibility equations. This means that removing gravitational forces the body would be residually stressed. In general, the final state of stress in an accreted body depends on the way in which new material was added and the state of stress (and loading) during accretion.

Kadish et al. [2005] extended the work of Brown and Goodman [1963] to a rotating solid sphere. Their approach consists of a decomposition of the stress tensor into a compatible time-dependent part and an incompatible time-independent part that represents the residual stresses that would remain if the external forces were removed after completion of accretion. This is obtained by a time integration of the Cauchy stress rate $\dot{\boldsymbol{\sigma}}$ (that is assumed to be compatible) leading to the following additive decomposition of the total stress:

$$
\boldsymbol{\sigma}(x, t)=\boldsymbol{\sigma}^{c}(x, t)+\boldsymbol{\sigma}^{o}(x),
$$

where the first term is a time-dependent compatible stress field, and the second term is time independent and represents that part of the residual stress that does not depend on the time of unloading. Since the timeindependent part does not satisfy the Beltrami-Michell compatibility equations, the problem is underdetermined. However, all the unknowns can be calculated by imposing some additional boundary conditions on the accretion surface that depend on the nature of the accretion process. For example, when the accreted material is stressfree at the time of attachment, all the components of the stress tensor - and not just the traction vector - are zero. They obtained a closed-form solution for this problem and used it to investigate the failure mechanisms in accreted planets.

Solidification is another phenomenon in which surface growth is relevant. Schwerdtfeger et al. [1998] presented a linear model of solidification in a round continuous casting mold (assuming a state of generalized plane strain with cylindrical symmetry) in order to calculate the residual stresses and investigate the formation of an air gap between the solidified metal and the mold. They explicitly highlighted the fact that in bodies that are subject to surface growth, e.g, solidifying bodies, there is no stress-free reference state for the body. ${ }^{2}$ All the equations were written in a rate form and the solution was integrated numerically using the known initial conditions at the moment of solidification, following therefore a method that is basically analogous to the one used by Brown and Goodman [1963].

Most of the existing works on surface growth that solve specific examples are restricted to small strains. Metlov [1985] presented a finite deformation theory of viscoelastic aging solids subject to accretion and discussed a couple of applications. The reason for considering aging and creep is that actual accretion processes usually take a considerable amount of time and hence these phenomena become important. In his description each particle is marked by a triplet $\left(\tau^{*}, v^{1}, v^{2}\right)$, where $\tau^{*}$ is a time of attachment and $\left(v^{1}, v^{2}\right)$ are coordinates on the growth surface. Using convected coordinates, he constructed for each point a deformation gradient as a composition of the prehistory of deformation and the deformation between the time of addition and the current time. There are many other works on the mechanics of surface growth (some of which use a geometric framework) by Russian researchers [Arutyunyan et al., 1990; Manzhirov, 1995; Lychev and Manzhirov, 2013a; Manzhirov, 2014; Lychev and Manzhirov, 2013a,b; Lychev, 2011]. Most of the recent works are formal. An exception is the work of Drozdov [1998a,b] who considers three configurations for an accreting body: reference, natural, and actual. These are local configurations with the natural configuration being stress free. His formulation is motivated by the multiplicative decomposition of the deformation gradient into elastic and plastic parts in finite plasticity. For nonlinear viscoelastic solids he models continuous accretion as a limit of discrete accretions and solves a few interesting specific examples.

In the biomechanics literature, Skalak et al. [1997] discussed the kinematics of surface growth for biological applications, e.g., growth of horns and seashells. They used the concept of generating cells, the material points defining the accretion surface that generate new material along an arbitrary curve that is not necessarily normal to the accretion surface. Ong and O'Reilly [2004] discussed surface growth in the context of rigid body dynamics. They first formulated the kinematics of a deformable body undergoing surface growth using

\footnotetext{
${ }^{2}$ This is true, however, as we will show in this paper, one can find a stress-free reference configuration that is a Riemannian manifold with a metric that depends on the accretion process. The material manifold, in general, is not flat and hence cannot be realized in the Euclidean space. In other words, the material manifold is incompatible with the geometry of the Euclidean ambient space.
} 
convected coordinates, although with a restrictive global decomposition of motion into elastic deformation and surface growth. Then they assumed that the body is rigid and obtained some modified kinematics and balance laws for accreting rigid bodies. As an example, they solved the problem of a cylinder rolling on an inclined plane while it is accumulating mass. Hodge and Papadopoulos [2010] proposed a theory of surface growth that they considered to be a continuum theory. They freeze the motion of a body at a time $\tau$ and postulate that going from $\tau$ to $\tau+t$, where $t$ is a small enough time increment, motion can be globally decomposed into an elastic deformation resulting in an intermediate configuration followed by a surface growth motion. In this theory they have to worry about extensions of different fields into the accreted region. The main issue with this theory is that it is, unfortunately, not a continuum theory; it is more or less an incremental theory with some questionable postulates.

In bulk growth of a body, one is given a fixed set of material points. Bulk growth changes the local stressfree configuration of the body at any point. This can be modeled by a Riemannian metric that depends on the growth distribution [Yavari, 2010]. In surface growth the situation is different; here, the set of material points is not fixed. In an accretion process, new material points may be added to or some existing material points may be removed from the boundary of the body in its current configuration. In this paper, instead of trying to formulate a general nonlinear theory of surface growth, we solve a few particular examples with cylindrical and spherical symmetries in the setting of nonlinear elasticity. We believe that this will pave the road for developing a general nonlinear theory of surface growth that we will discuss in a future communication. We consider axisymmetric accretion of cylindrical and spherical bodies, i.e. we start from a cylinder or sphere that at any instant of time is a cylinder or a sphere throughout the process of accretion. Our model problem is a hollow cylinder or a hollow spherical ball that is under a time-dependent internal pressure. The loaded body undergoes an accretion process in which stress-free (or pre-stressed) particles are continuously attached to the body. Unloading the accreted body at any instant of time the body is residually stressed, in general. In this paper, we are interested in deriving the governing equations of a nonlinear elastic body going through this special type of surface growth. In our geometric framework, material manifold is time-dependent and has a Riemannian metric that depends on the surface growth characteristics and the loading and deformation history during accretion.

This paper is organized as follows. In $\S 2$, we tersely review some elements of Riemannian geometry and the geometric theory of anelasticity and discuss some aspects of nonlinear surface growth mechanics in a geometric framework. In $\S 3$ we analyze, as a prelude to continuous growth, a thick hollow cylinder that is deformed under an internal pressure and while deformed is accreted by a single layer of stress-free material. We find the material metric and show that it depends on the external loads during the accretion process and the thickness of the added layer. We then formulate the continuous analogue of this problem in $\S 4$. We consider a thick hollow cylinder under a time-dependent internal pressure while undergoing a symmetric and continuous accretion on its outer boundary where the added material is assumed to be stress-free (or pre-stressed) at the time of attachment to the body (outer accretion). We next assume that the growth surface is the inner boundary of the cylinder (inner accretion), i.e. material is continuously added to the body on its inner boundary (the added material is under a state of hydrostatic pressure or suction at the time of attachment). We linearize the nonlinear governing equations and show that the accretion stresses are identical to those calculated using the linear surface growth theory of Brown and Goodman [1963]. In $\S 5$ we study the outer accretion problem for a hollow thick spherical ball. In $\S 6$, we numerically solve the governing equations of accreting cylindrical bars and spherical balls in the case of an incompressible neo-Hookean solid. In particular, we calculate the residual stresses and demonstrate the anelastic nature of accretion. Conclusions are given in $\S 7$.

\section{Geometric Anelasticity and the Mechanics of Growth}

In this section, we tersely review some elements of Riemannian geometry and the geometric theory of nonlinear elasticity and anelasticity. For more detailed discussions, see [Marsden and Hughes, 1983; Yavari, 2010; Yavari and Goriely, 2012a].

Riemannian geometry. For a smooth $n$-manifold $\mathcal{M}$, the tangent space to $\mathcal{M}$ at a point $p \in \mathcal{M}$ is denoted $T_{p} \mathcal{M}$. Suppose $\mathcal{N}$ is another $n$-manifold and $\psi: \mathcal{M} \rightarrow \mathcal{N}$ is a smooth and invertible map. A smooth vector field $\mathbf{W}$ on $\mathcal{M}$ assigns a vector $\mathbf{W}_{p}$ to every $p \in \mathcal{M}$ and $p \mapsto \mathbf{W}_{p} \in T_{p} \mathcal{M}$ varies smoothly. If $\mathbf{W}$ is a vector field 
on $\mathcal{M}$, then $\psi_{*} \mathbf{W}=T \psi \cdot \mathbf{W} \circ \psi^{-1}$ is a vector field on $\psi(\mathcal{M})$ called the push-forward of $\mathbf{W}$ by $\psi$. Similarly, if $\mathbf{w}$ is a vector field on $\psi(\mathcal{M}) \subset \mathcal{N}$, then $\psi^{*} \mathbf{w}=T\left(\psi^{-1}\right) \cdot \mathbf{w} \circ \psi$ is a vector field on $\mathcal{M}$ that is called the pull-back of $\mathbf{w}$ by $\psi$. Let us denote $\mathbf{F}=T \psi$. In the local charts $\left\{X^{A}\right\}$ and $\left\{x^{a}\right\}$ for $\mathcal{M}$ and $\mathcal{N}$, respectively, $\mathbf{F}$ (a two-point tensor) has the following representation (when $\psi$ is a deformation mapping, $\mathbf{F}$ is the so-called deformation gradient of nonlinear elasticity)

$$
\mathbf{F}=F^{a}{ }_{A} \frac{\partial}{\partial X^{A}} \otimes d x^{a}, \quad F^{a}{ }_{A}=\frac{\partial \psi^{a}}{\partial X^{A}},
$$

where $\left\{\frac{\partial}{\partial X^{A}}\right\}$ is a basis for $T_{p} \mathcal{M}$ and $\left\{d x^{a}\right\}$ is a basis for $T_{\psi(p)}^{*} \psi(\mathcal{M})$, the co-tangent space, i.e. the dual space of $T_{\psi(p)} \psi(\mathcal{M})$, or the space of co-vectors (1-forms). The push-forward and pull-back of vectors have the following coordinate representations

$$
\left(\psi_{*} \mathbf{W}\right)^{a}=F^{a}{ }_{A} W^{A}, \quad\left(\psi^{*} \mathbf{w}\right)^{A}=\left(F^{-1}\right)_{a}{ }^{A} w^{a} .
$$
$\mathcal{M}$

A type $\left(\begin{array}{l}0 \\ 2\end{array}\right)$-tensor at $p \in \mathcal{M}$ is a bilinear map $\mathbf{T}: T_{p} \mathcal{M} \times T_{p} \mathcal{M} \rightarrow \mathbb{R}$. In a local coordinate chart $\left\{X^{A}\right\}$ for

$$
\mathbf{T}(\mathbf{U}, \mathbf{W})=T_{A B} U^{A} W^{B}
$$

where $\mathbf{U}, \mathbf{W} \in T_{p} \mathcal{M}$.

$(\mathcal{M}, \mathbf{G})$ is a Riemannian manifold if $\mathcal{M}$ is a smooth manifold equipped with an inner product $\mathbf{G}_{p}$ on the tangent space $T_{p} \mathcal{M}$ that varies smoothly in the sense that if $\mathbf{U}$ and $\mathbf{W}$ are vector fields on $\mathcal{M}$, then

$$
p \mapsto \mathbf{G}_{p}\left(\mathbf{U}_{p}, \mathbf{W}_{p}\right)=:\left\langle\left\langle\mathbf{U}_{p}, \mathbf{W}_{p}\right\rangle\right\rangle_{\mathbf{G}_{p}},
$$

is a smooth function. Obviously, $\mathbf{G}$ is a symmetric $\left(\begin{array}{l}0 \\ 2\end{array}\right)$-tensor field on $\mathcal{M}$.

Suppose $(\mathcal{M}, \mathbf{G})$ and $(\mathcal{N}, \mathbf{g})$ are Riemannian manifolds and $\psi: \mathcal{M} \rightarrow \mathcal{N}$ is a diffeomorphism (smooth map with smooth inverse). Push-forward of the metric $\mathbf{G}$ is a metric on $\psi(\mathcal{M}) \subset \mathcal{N}$, which is denoted by $\psi_{*} \mathbf{G}$ and is defined as

$$
\left(\psi_{*} \mathbf{G}\right)_{\psi(p)}\left(\mathbf{u}_{\psi(p)}, \mathbf{w}_{\psi(p)}\right):=\mathbf{G}_{p}\left(\left(\psi^{*} \mathbf{u}\right)_{p},\left(\psi^{*} \mathbf{w}\right)_{p}\right) .
$$

In components

$$
\left(\psi_{*} \mathbf{G}\right)_{a b}=\left(F^{-1}\right)_{a}^{A}\left(F^{-1}\right)_{b}^{B} G_{A B} .
$$

Similarly, pull-back of the metric $\mathbf{g}$ is a metric in $\psi^{-1}(\mathcal{N}) \subset \mathcal{M}$, which is denoted by $\psi^{*} \mathbf{g}$ and is defined as

$$
\left(\psi^{*} \mathbf{g}\right)_{p}\left(\mathbf{U}_{p}, \mathbf{W}_{p}\right):=\mathbf{g}_{\psi(p)}\left(\left(\psi_{*} \mathbf{U}\right)_{\psi(p)},\left(\psi_{*} \mathbf{W}\right)_{\psi(p)}\right)
$$

In components

$$
\left(\psi^{*} \mathbf{g}\right)_{A B}=F^{a}{ }_{A} F^{b}{ }_{B} g_{a b} .
$$

If $\mathbf{g}=\psi_{*} \mathbf{G}$, or equivalently, $\mathbf{G}=\psi^{*} \mathbf{g}, \psi$ is called an isometry and the Riemannian manifolds $(\mathcal{M}, \mathbf{G})$ and $(\mathcal{N}, \mathbf{g})$ are isometric. Note that an isometry preserves distances.

Kinematics. In nonlinear elasticity a body $B$ is identified with a Riemannian manifold $(\mathcal{B}, \mathbf{G})$. A configuration of $\mathcal{B}$ is a smooth embedding $\varphi: \mathcal{B} \rightarrow \mathcal{S}$, where $(\mathcal{S}, \mathbf{g})$ is the ambient space manifold with a (static) background metric $\mathbf{g}$. In most applications the ambient space is assumed to be the Euclidean space. We denote by $\nabla^{\mathbf{G}}$ and $\nabla^{\mathbf{g}}$ the Levi-Civita connections associated with the Riemannian manifolds $(\mathcal{B}, \mathbf{G})$ and $(\mathcal{S}, \mathbf{g})$, respectively. The so-called deformation gradient $\mathbf{F}$ is the derivative map of $\varphi_{t}$ defined as

$$
\mathbf{F}(X, t)=d \varphi_{t}(X): T_{X} \mathcal{B} \rightarrow T_{\varphi_{t}(X)} \mathcal{S} .
$$

The adjoint of $\mathbf{F}$ is defined by

$$
\mathbf{F}^{\top}(X, t): T_{\varphi_{t}(X)} \mathcal{S} \rightarrow T_{X} \mathcal{B}, \quad\left\langle\langle\mathbf{F V}, \mathbf{v}\rangle_{\mathbf{g}}=\left\langle\left\langle\mathbf{V}, \mathbf{F}^{\top} \mathbf{v}\right\rangle_{\mathbf{G}}, \quad \forall \mathbf{V} \in T_{X} \mathcal{B}, \mathbf{v} \in T_{\varphi_{t}(X)} \mathcal{S},\right.\right.
$$

where $\langle\langle,\rangle\rangle_{\mathbf{g}}$ and $\left\langle\langle,\rangle_{\mathbf{G}}\right.$ are the inner products induced by $\mathbf{g}$ and $\mathbf{G}$, respectively. The right Cauchy-Green deformation tensor is defined as $\mathbf{C}(X, t)=\mathbf{F}^{\top}(X, t) \mathbf{F}(X, t): T_{X} \mathcal{B} \rightarrow T_{X} \mathcal{B}$. In the coordinate charts $\left\{X^{A}\right\}$ and 
$\left\{x^{a}\right\}$ for $\mathcal{B}$ and $\mathcal{S}$, respectively, $\mathbf{C}$ is written as: $C^{A}{ }_{B}=G^{A M} F^{a}{ }_{M} F^{b}{ }_{B} g_{a b}$. The Jacobian of the motion $J$ relates the material and spatial Riemannian volume elements $d V(X, \mathbf{G})$ and $d v(x, \mathbf{g})$ by $d v=J d V$ and is given as

$$
J=\sqrt{\frac{\operatorname{det} \mathbf{g}}{\operatorname{det} \mathbf{G}}} \operatorname{det} \mathbf{F} .
$$

In classical nonlinear elasticity, a motion is a time-dependent map $\varphi_{t}: \mathcal{B} \rightarrow \mathcal{S}$, from a fixed material manifold $(\mathcal{B}, \mathbf{G})$ into an ambient space manifold $(\mathcal{S}, \mathbf{g})$. In the presence of surface growth, $\mathcal{B}$ is no longer fixed as material points are added to or removed from the boundary of the body in its current configuration. For an accreting body $\varphi_{t}: \mathcal{B}_{t} \rightarrow \mathcal{S}$, where $\left(\mathcal{B}_{t}, \mathbf{G}\right)$ is a time-dependent material manifold. The metric $\mathbf{G}$ explicitly depends on the history of loading and deformation during accretion as we will see shortly in the examples of cylindrical and spherical growing bodies.

In an accretion process new material is added to or existing material is removed from the growth surface $\omega_{t}$, which is part of the boundary of the body in its deformed configuration, i.e. $\omega_{t} \subset \varphi_{t}\left(\partial \mathcal{B}_{t}\right)$. The growth surface in the material manifold is denoted by $\Omega_{t}$. For any $X \in \mathcal{B}_{t}$ we define a time of attachment $\tau(X) \leq t$. For $X \in \mathcal{B}_{0}$ (the initial body), $\tau(X)=0$. Note that

$$
\Omega_{t}=\left\{X \in \mathcal{B}_{t}: \tau(X)=t\right\}
$$

Constitutive equations. In this paper we restrict our calculations to incompressible isotropic hyperelastic solids. The left Cauchy-Green deformation tensor $\mathbf{B}^{\sharp}=\varphi^{*}\left(\mathbf{g}^{\sharp}\right)$ has components $B^{A B}=\left(F^{-1}\right)^{A}{ }_{a}\left(F^{-1}\right)^{B}{ }_{b} g^{a b}$, where $g^{a b}$ are components of $\mathbf{g}^{\sharp}$. The spatial analogues of $\mathbf{C}^{b}$ and $\mathbf{B}^{\sharp}$ are

$$
\mathbf{c}^{\mathrm{b}}=\varphi_{*}(\mathbf{G}), c_{a b}=\left(F^{-1}\right)^{A}{ }_{a}\left(F^{-1}\right)^{B}{ }_{b} G_{A B}, \text { and } \mathbf{b}^{\sharp}=\varphi_{*}\left(\mathbf{G}^{\sharp}\right), b^{a b}=F^{a}{ }_{A} F^{b}{ }_{B} G^{A B} \text {. }
$$

$\mathbf{b}^{\sharp}$ is called the Finger deformation tensor. Note that $\mathbf{C}$ and $\mathbf{b}$ have the same principal invariants, which we denote by $I_{1}, I_{2}$, and $I_{3}$ [Ogden, 1984]. For an isotropic solid the energy function $W$ depends only on $I_{1}, I_{2}$, and $I_{3}$. It is seen that, for example, $I_{1}=\operatorname{tr} \mathbf{b}=F^{a}{ }_{A} F^{b}{ }_{B} G^{A B} g_{a b}$ explicitly depends on the material metric. For an incompressible $\left(I_{3}=1\right)$ and isotropic hyperelastic solid with energy function $W=W\left(I_{1}, I_{2}\right)$, the Cauchy stress has the following classic representation [Simo and Marsden, 1984]

$$
\boldsymbol{\sigma}=\left(-p+2 I_{2} \frac{\partial W}{\partial I_{2}}\right) \mathbf{g}^{\sharp}+2 \frac{\partial W}{\partial I_{1}} \mathbf{b}^{\sharp}-2 \frac{\partial W}{\partial I_{2}} \mathbf{b}^{-1},
$$

where $p$ is the Lagrange multiplier associated with the internal incompressibility constraint $J=1$. It should be emphasized that the Cauchy stress explicitly depends on the material metric G. It should also be emphasized that we assume that the residually-stressed body is isotropic in its stress-free material manifold. In the current configuration the body may not be isotropic. We assume that a growing body is initially stress-free (the initial body is assumed stress free) and isotropic and that the added material is isotropic in its stress-free configuration.

Equilibrium equations. As surface growth is a slow process, one can ignore the inertial effects. The local balance of linear momentum in terms of the Cauchy stress tensor reads

$$
\operatorname{div} \boldsymbol{\sigma}+\rho \mathbf{f}=\mathbf{0}
$$

where $\mathbf{f}$ is the body force per unit deformed mass and div denotes the spatial divergence operator, which in components reads

$$
(\operatorname{div} \boldsymbol{\sigma})^{a}=\sigma_{\mid b}^{a b}=\frac{\partial \sigma^{a b}}{\partial x^{b}}+\sigma^{a c} \gamma_{c b}^{b}+\sigma^{c b} \gamma_{c b}^{a},
$$

where $\gamma_{b c}^{a}$ is the Christoffel symbol of the Levi-Civita connection $\nabla^{\mathbf{g}}$ in the local chart $\left\{x^{a}\right\}$, defined as $\nabla^{\mathbf{g}} \partial_{b} \partial_{c}=\gamma_{b c}^{a} \partial_{a}$, (similarly, for the material manifold $\left.\nabla^{\mathbf{G}_{\partial_{B}}} \partial_{C}=\Gamma^{A}{ }_{B C} \partial_{A}\right)$. 
The physical significance of a material manifold. In classical nonlinear elasticity one starts with a reference configuration that is assumed to be stress free. Motion is then a time-dependent mapping from the reference configuration into an Euclidean ambient space. In anelasticity (in the sense of Eckart [1948]), the body is residually stressed and hence there exists no global stress-free reference configuration, in general, that can be realized in the Euclidean ambient space. One may however, define local relaxed configurations using a multiplicative decomposition of deformation gradient. A global stress-free configuration cannot be isometrically embedded in the Euclidean ambient space, in general, due to its non-Euclidean geometry. In other words, a global stress-free configuration of the body is incompatible with the geometry of the Euclidean ambient space. This idea was introduced in the mechanics of defects independently by Kondo [1955a,b] and Bilby et al. [1955]. In a geometric formulation of anelasticity the material manifold has a geometry that depends on the source of anelasticity. In the case of point and line defects the geometry depends on the (area or volume) density of defects [Yavari and Goriely, 2012a,b, 2013a, 2014]. In thermoelasticity, the material metric depends on both the temperature distribution and the thermal properties of the solid, e.g. (temperature-dependent) coefficient of thermal expansion [Ozakin and Yavari, 2010; Sadik and Yavari, 2016b]. In bulk growth material metric is explicitly time-dependent and is a function of the mass flux through the balance of mass [Yavari, 2010; Sadik et al., 2016]. For inclusions (or inhomogeneities with eigenstrains) material metric explicitly depends on the distribution of (finite) eigenstrains [Yavari and Goriely, 2013b, 2015; Golgoon et al., 2016]. After solving the problem of surface growth of an infinitely-long cylindrical bar (or a hollow spherical ball) we will see that, as expected, the material manifold is time-dependent and has a metric that is determined by the history of loading and deformation during surface growth. Interestingly, neither the material manifold nor the material metric is unique. We will see that there are infinitely many isometric Riemannian material manifolds that are all equivalent in the sense that stress (and particularly residual stress) does not depend on the particular choice of a material manifold from this equivalence class. In the material manifold the growing body is stress-free by construction. Having a material manifold a nonlinear surface growth problem is transformed into a classical nonlinear elasticity problem as long as the non-trivial geometry of the material manifold is taken into account properly. We will demonstrate this idea for accreting cylindrical and spherical bodies in this paper.

\section{Attachment of a Stress-Free Layer to a Deformed Infinitely Long Thick Hollow Cylinder}

In this section we formulate the boundary-value problem of the attachment of a single stress-free layer on the outer boundary of a deformed hollow cylinder. The motivation for studying this problem is to gain some insight on the effect of a single accretion layer on the stress-free material manifold of the body. The calculations of this section can be viewed as a prelude to the discussion of continuous surface growth in $\S 4$.

We consider a stress-free and infinitely long hollow cylindrical bar with inner and outer radii $R_{1}$ and $R_{2}$, respectively, subject to an internal pressure $p_{i}$, which is specified (see Fig.1(a)). In the deformed configuration (Fig.1(b)) the inner and outer radii are $r_{1}$ and $r_{2}$, respectively. While keeping the internal pressure a layer of stress-free material (for the sake of simplicity we assume the same material ${ }^{3}$ ) of thickness $\Delta$ is added to the outer boundary of the body through surface growth (accretion) (see Fig.1(c)). If the internal pressure is removed the accreted body would be residually stressed (see Fig.1(d)). Note that this problem is very similar to the classical shrink-fit problem [Antman and Shvartsman, 1994] and can be solved using standard methods of nonlinear elasticity. Here we present an alternative approach that will be useful in a systematic analysis of continuous surface growth that will be discussed in the next section. The idea is to find a Riemannian material manifold in which the accreted body is stress free. Note that such a material manifold may be incompatible with the geometry of the Euclidean ambient space. This idea is schematically described in Fig.2. Only the configurations shown in Figs.2(b) and (c) are physically observable. Fig.2(b) shows the loaded body with a layer of stress-free material added to it and Fig.2(c) depicts the residually-stressed accreted body after it is unloaded. In Fig.2(a) we show the initial stress-free body and the stress-free layer that will be attached to it after the initial body is deformed. Note that the stress-free configurations in the Euclidean ambient space are detached, i.e., a global stress-free configuration is incompatible with the geometry of the Euclidean ambient space and this is why the accreted body will be residually-stressed after the removal of external forces (here the

\footnotetext{
${ }^{3}$ By the "same material" we mean that the undeformed hollow cylinder and the stress-free added layer have the same energy functions.
} 


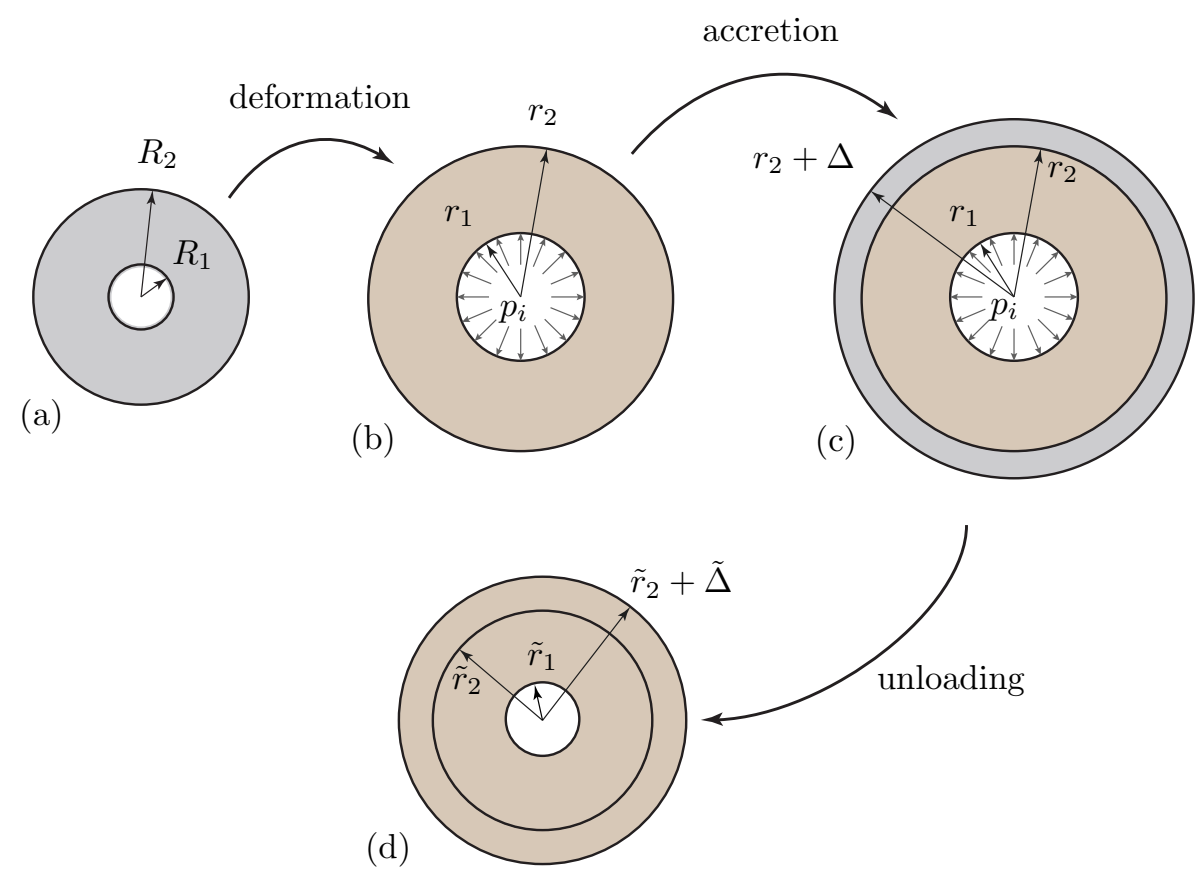

Figure 1: Discrete Accretion: (a) An annulus (annular cylinder) of inner and outer radii $R_{1}$ and $R_{2}$, respectively, in its stress-free configuration in the Euclidean ambient space. (b) The deformed annulus under a specified internal pressure $p_{i}$. (c) The deformed annulus with a layer of stress-feee material of thickness $\Delta$ attached to its outer boundary. (d) The residually-stressed accreted body after removal of the external forces (here the specified internal pressure $p_{i}$ ).

internal pressure $p_{i}$ ). Next we will construct a connected Riemannian manifold in which the accreted body is stress-free by construction (Fig.2(d)).

\subsection{Construction of a material manifold}

As the cylindrical bar is infinitely long we can model it as a 2D annulus with polar coordinates $(R, \Theta)$ and $(r, \theta)$ in the reference and current configurations, respectively. The ambient space is equipped with the flat Euclidean metric that in polar coordinates reads ${ }^{4}$

$$
\mathbf{g}=\left(\begin{array}{cc}
1 & 0 \\
0 & r^{2}
\end{array}\right)
$$

The (disconnected) Euclidean stress-free configuration shown in Fig.2(a) is the union of two sets, i.e., $\mathcal{B}_{0}=$ $\mathcal{B}_{0}^{-} \cup \mathcal{B}_{0}^{+}$, where

$$
\mathcal{B}_{0}^{-}=\left\{(R, \Theta): R_{1} \leq R \leq R_{2}, 0 \leq \Theta<2 \pi\right\}, \quad \mathcal{B}_{0}^{+}=\left\{(R, \Theta): r_{2} \leq R \leq r_{2}+\Delta, 0 \leq \Theta<2 \pi\right\} .
$$

Here, we know that

$$
\mathbf{G}_{0}^{-}=\mathbf{G}_{0}^{+}=\left(\begin{array}{cc}
1 & 0 \\
0 & R^{2}
\end{array}\right) .
$$

Let us consider the following maps. The deformation of the initial hollow cylinder loaded by an internal pressure $p_{i}$ is denoted by $\psi_{0}^{-}: \mathcal{B}_{0}^{-} \rightarrow \mathcal{S}$. The trivial map (identity map) of the stress-free accreted layer into itself is denoted by $\psi_{0}^{+}: \mathcal{B}_{0}^{+} \rightarrow \mathcal{S}$. The connected material manifold is denoted by $\mathcal{B}=\mathcal{B}^{-} \cup \mathcal{B}^{+}$and is shown in Fig.2(d) where

$$
\mathcal{B}^{-}=\left\{(R, \Theta): R_{1} \leq R \leq R_{2}, 0 \leq \Theta<2 \pi\right\}, \quad \mathcal{B}^{+}=\left\{(R, \Theta): R_{2} \leq R \leq R_{2}+\Delta^{\prime}, 0 \leq \Theta<2 \pi\right\} .
$$

\footnotetext{
${ }^{4}$ This simply means that the square of the distance between two points with coordinates $(r, \theta)$ and $(r+d r, \theta+d \theta)$ is $d s^{2}=$ $d r^{2}+r^{2} d \theta^{2}$
} 


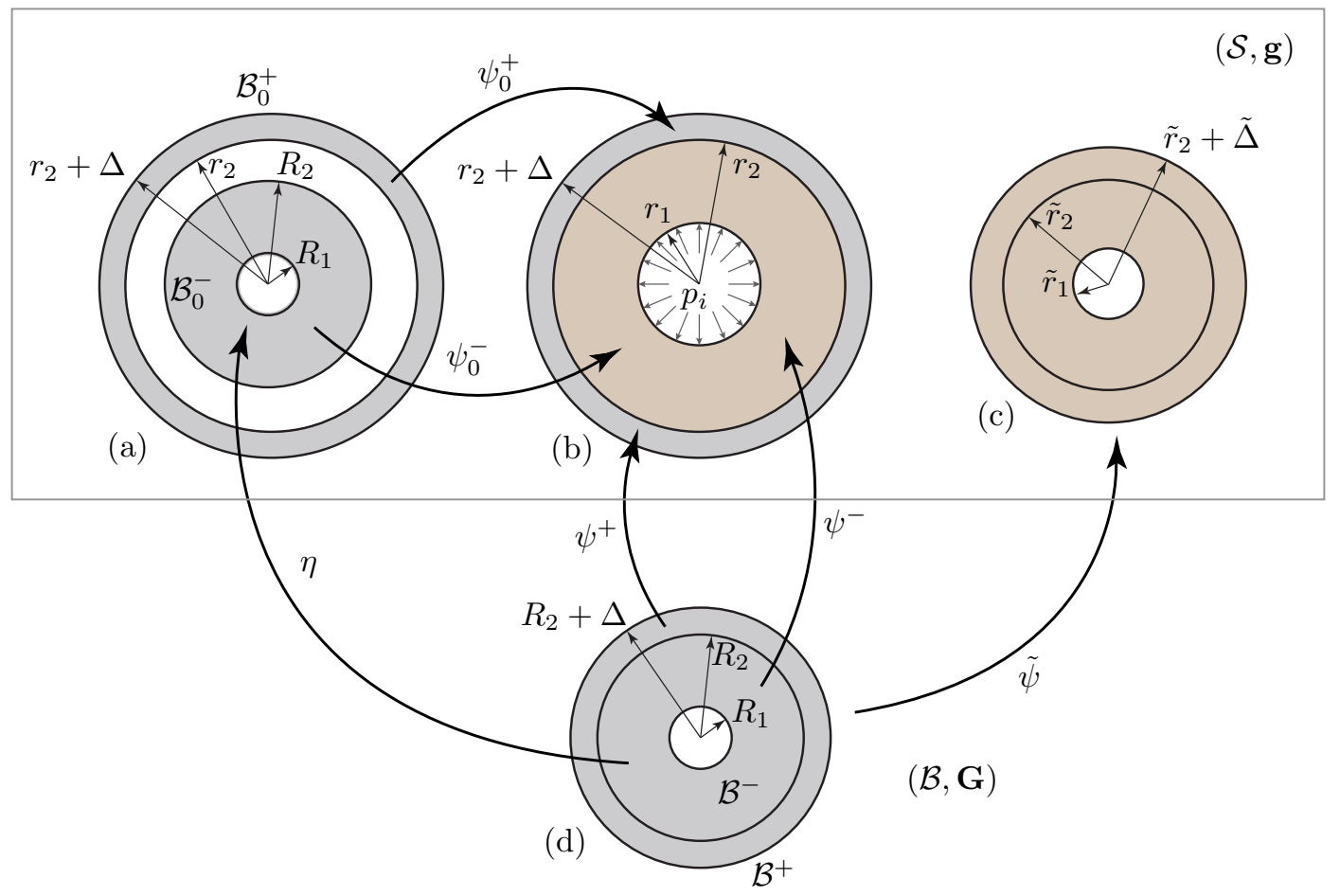

Figure 2: Discrete Accretion: (a) Stress-free configurations of the initial body and the accreted layer in the Euclidean ambient space $\mathcal{S}$. (b) Loaded body with a layer of stress-free material added to its outer boundary. (c) Residually-stressed accreted body after removal of the external forces. (d) Riemannian material manifold $(\mathcal{B}, \mathbf{G})$ of the accreted body. Note that the configurations (a), $(b)$, and $(c)$ lie in the Euclidean ambient space $(\mathcal{S}, \mathbf{g})$. In the incompatible Riemannian material manifold there are no residual stresses.

$\Delta^{\prime}>0$ has not been specified; any positive value is admissible. A convenient choice is $\Delta^{\prime}=\Delta$. Deformation mapping from the material manifold into the loaded configuration (Fig.2(b)) is denoted by $\psi: \mathcal{B} \rightarrow \mathcal{S}$. We define $\psi_{0}^{-}=\left.\psi\right|_{\mathcal{B}_{0}^{-}}$and $\psi_{0}^{+}=\left.\psi\right|_{\mathcal{B}_{0}^{+}}$. These maps must satisfy the following properties:

$$
\psi^{-}\left(\mathcal{B}^{-}\right)=\psi_{0}^{-}\left(\mathcal{B}_{0}^{-}\right), \quad \psi^{+}\left(\mathcal{B}^{+}\right)=\psi_{0}^{+}\left(\mathcal{B}_{0}^{+}\right) .
$$

They have the coordinate representations

$$
\psi_{0}(R, \Theta)=\left(r_{0}(R), \Theta\right), \quad \psi(R, \Theta)=(r(R), \Theta) .
$$

Note that $\psi^{-}(R, \Theta)=(r(R), \Theta)$ can be calculated using the governing equations of nonlinear elasticity as will be explained in the following and for $\psi^{+}(R, \Theta)=(r(R), \Theta)$ any differentiable $r(R)$ that satisfies $r\left(R_{2}\right)=r_{2}$ and $r\left(R_{2}+\Delta^{\prime}\right)=r_{2}+\Delta$ is admissible. The material manifold $(\mathcal{B}, \mathbf{G})$ is isomorphic to the disconnected Euclidean stress-free configuration, i.e. $\left(\mathcal{B}_{0}^{-}, \mathbf{G}_{0}^{-}\right)$and $\left(\mathcal{B}^{-}, \mathbf{G}^{-}\right)$are isometric and the isometry map is $\eta=\left(\psi_{0}^{-}\right)^{-1} \circ \psi^{-}$: $\mathcal{B}^{-} \rightarrow \mathcal{B}_{0}^{-}$. Similarly, $\left(\mathcal{B}_{0}^{+}, \mathbf{G}_{0}^{+}\right)$and $\left(\mathcal{B}^{+}, \mathbf{G}^{+}\right)$are isometric. However, knowing that the accreted layer in the deformed configuration is stress-free, equivalently, $\left(\mathcal{B}^{+}, \mathbf{G}^{+}\right)$is isometric to $\left(\psi_{0}^{+}\left(\mathcal{B}_{0}^{+}\right), \mathbf{g}\right)$. In summary we have

i) $\left(\mathcal{B}^{-}, \mathbf{G}^{-}\right)$and $\left(\mathcal{B}_{0}^{-}, \mathbf{G}_{0}^{-}\right)$are isometric and hence

$$
\mathbf{G}^{-}=\eta^{*} \mathbf{G}_{0}^{-}=\left(\left(\psi_{0}^{-}\right)^{-1} \circ \psi^{-}\right)^{*} \mathbf{G}_{0}^{-}=\left(\psi^{-}\right)^{*} \circ\left(\psi_{0}^{-}\right)_{*} \mathbf{G}_{0}^{-} .
$$

ii) $\left(\mathcal{B}^{+}, \mathbf{G}^{+}\right)$and $\left(\psi_{0}^{+}\left(\mathcal{B}_{0}^{+}\right), \mathbf{g}\right)$ are isometric and thus

$$
\mathbf{G}^{+}=\left(\psi^{+}\right)^{*} \mathbf{g} .
$$


Therefore, we have

$$
\begin{aligned}
R_{1} \leq R \leq R_{2}: & \mathbf{G}^{-}=\left(\begin{array}{cc}
{\eta^{\prime}}^{2}(R) & 0 \\
0 & \eta^{2}(R)
\end{array}\right), \\
R_{2} \leq R \leq R_{2}+\Delta^{\prime}: & \mathbf{G}^{+}=\left(\begin{array}{cc}
{r^{\prime}}^{2}(R) & 0 \\
0 & r^{2}(R)
\end{array}\right),
\end{aligned}
$$

where $\Delta^{\prime}$ is such that $r\left(R_{2}+\Delta^{\prime}\right)=r_{2}+\Delta$. Choosing $\Delta^{\prime}=\Delta,{ }^{5}$ any differentiable function $\eta(R)$ that satisfies $\eta\left(R_{1}\right)=R_{1}$ and $\eta\left(R_{2}\right)=R_{2}$ is admissible. We will show that the stress distribution is independent of the choice of an admissible $\eta$. In particular, for the sake of simplicity we choose $\eta(R)=R$, i.e., $r(R)=r_{0}(R)$, meaning that $\mathcal{B}^{-}$is trivially given by $\mathcal{B}_{0}^{-}$.

Calculation of the map $\psi^{-}(R, \Theta)=\psi_{0}^{-}(R, \Theta)=\left(r_{0}(R), \Theta\right)=(r(R), \Theta)$ and the outer radius of the deformed initial body $r_{2}$. Next we calculate the configuration $r(R)$ and the corresponding stress distribution in the region $R_{1} \leq R \leq R_{2}$ when the internal pressure $p_{i}$ is being applied. Let us assume that the initial annulus and the accreted layer are both made of the same incompressible isotropic solid (in their stress-free states) with energy function $W=W\left(I_{1}, I_{2}\right)$. The material metric is $\mathbf{G}=\operatorname{diag}\left\{1, R^{2}\right\}$, and the deformation gradient is $\mathbf{F}=\operatorname{diag}\left\{r^{\prime}(R), 1\right\}$. Therefore, the incompressibility condition reads

$$
J=\sqrt{\frac{\operatorname{det} \mathbf{g}}{\operatorname{det} \mathbf{G}}} \operatorname{det} \mathbf{F}=\frac{r(R) r^{\prime}(R)}{R}=1,
$$

from which, denoting $r_{2}=r\left(R_{2}\right)$, one obtains

$$
r^{2}(R)=R^{2}-R_{2}^{2}+r_{2}^{2}
$$

Using (2.13), the Finger tensor reads

$$
\mathbf{b}^{\sharp}=\left(\begin{array}{cc}
\frac{R^{2}}{r^{2}(R)} & 0 \\
0 & \frac{1}{R^{2}}
\end{array}\right) \text {. }
$$

The principal invariants of $\mathbf{b}$ are

$$
I_{1}=\frac{r^{2}(R)}{R^{2}}+\frac{R^{2}}{r^{2}(R)}, \quad I_{2}=1
$$

Note that $\left(b^{-1}\right)^{a b}=c^{a b}=g^{a m} g^{b n} c_{m n}$ and thus

$$
\mathbf{b}^{-1}=\left(\begin{array}{cc}
\frac{r^{2}(R)}{R^{2}} & 0 \\
0 & \frac{R^{2}}{r^{4}(R)}
\end{array}\right) .
$$

The Cauchy stress is written as $\boldsymbol{\sigma}=\left(-p+I_{2} \beta\right) \mathbf{g}^{\sharp}+\alpha \mathbf{b}^{\sharp}-\beta \mathbf{b}^{-1}$, where $\alpha=2 \frac{\partial W}{\partial I_{1}}$ and $\beta=2 \frac{\partial W}{\partial I_{2}}$, and in polar coordinates it reads

$$
\boldsymbol{\sigma}(R)=\left(\begin{array}{cc}
-p(R)+\frac{R^{2}}{r^{2}(R)} \alpha(R)+\left(1-\frac{r^{2}(R)}{R^{2}}\right) \beta(R) & 0 \\
0 & -\frac{p(R)}{r^{2}(R)}+\frac{\alpha(R)}{R^{2}}+\left(1-\frac{R^{2}}{r^{2}(R)}\right) \frac{\beta(R)}{r^{2}(R)}
\end{array}\right) .
$$

The only non-trivial equilibrium equation is the the radial equilibrium equation that is written as

$$
\frac{\partial \sigma^{r r}}{\partial r}+\frac{1}{r} \sigma^{r r}-r \sigma^{\theta \theta}=0
$$

This is simplified to read

$$
\frac{d \sigma^{r r}}{d R}=\left[1-\frac{R^{4}}{r^{4}(R)}\right] \frac{\alpha(R)+\beta(R)}{R} .
$$

We know that $\sigma^{r r}\left(R_{1}\right)=-p_{i}$. Thus, integrating the above ODE from $R_{1}$ to $R$, one obtains

$$
\sigma^{r r}(R)=-p_{i}+\int_{R_{1}}^{R} \frac{\alpha(x)+\beta(x)}{x}\left[1-\frac{x^{4}}{r^{4}(x)}\right] d x .
$$

\footnotetext{
${ }^{5}$ We will work with a similar choice in the continuous accretion case in the next section.
} 
We know that $\sigma^{r r}\left(R_{2}\right)=0$, and therefore

$$
\int_{R_{1}}^{R_{2}} \frac{\alpha(x)+\beta(x)}{x}\left[1-\frac{x^{4}}{r^{4}(x)}\right] d x=p_{i}
$$

which determines the unknown $r_{2}$ in (3.11).

Calculation of the map $\psi^{+}(R, \Theta)$. For the outer layer $\mathcal{B}^{+}$for which $R_{2} \leq R \leq R_{2}+\Delta^{\prime}$ any differentiable $\operatorname{map} \psi^{+}: \mathcal{B}^{+} \rightarrow \mathcal{S}$ that satisfies

$$
\psi^{+}\left(R_{2}, \Theta\right)=\left(r_{2}, \Theta\right), \quad \psi^{+}\left(R_{2}+\Delta^{\prime}, \Theta\right)=\left(r_{2}+\Delta, \Theta\right), \quad \Theta \in[0,2 \pi),
$$

is admissible. Choosing $\Delta^{\prime}=\Delta$, the simplest choice for $\psi^{+}$is $\psi^{+}(R, \Theta)=\left(R-R_{2}+r_{2}, \Theta\right)$, i.e.

$$
r(R)=R-R_{2}+r_{2}, \quad R_{2} \leq R \leq R_{2}+\Delta .
$$

Therefore, we have the following material metric tensors in the two layers:

$$
\begin{aligned}
R_{1} \leq R \leq R_{2}: & \mathbf{G}^{-}=\left(\begin{array}{cc}
1 & 0 \\
0 & R^{2}
\end{array}\right), \\
R_{2} \leq R \leq R_{2}+\Delta: & \mathbf{G}^{+}=\left(\begin{array}{cc}
1 & 0 \\
0 & \left(R-R_{2}+r_{2}\right)^{2}
\end{array}\right) .
\end{aligned}
$$

Notice that $G^{-\Theta \Theta}\left(R_{2}\right)=R_{2}{ }^{2} \neq r_{2}^{2}=G^{+\Theta \Theta}\left(R_{2}\right)$. This discontinuity of the material metric will induce residual stresses in the unloaded configuration of Fig.2(c).

\subsection{Calculation of residual stresses for an incompressible isotropic solid}

We now calculate the residual stress distribution when $p_{i}$ is removed. In this configuration the boundary between the initial body and the accreted (secondary) body is a circle (infinite cylinder) of unknown radius $\tilde{r}_{2}$. We denote the deformation from the reference configuration into the residually-stressed configuration by $(\tilde{r}, \tilde{\theta})=(\tilde{r}, \Theta)$ and hence $\tilde{r}_{2}=\tilde{r}\left(R_{2}\right)$, see Fig. 2 . Note that $\tilde{r}_{2} \neq r_{2}$, in general. We have the following boundary and continuity conditions: $\tilde{\sigma}^{r r}\left(R_{1}\right)=\tilde{\sigma}^{r r}\left(R_{2}+\Delta^{\prime}\right)=0, \tilde{\sigma}^{r r}\left(R_{2}^{-}\right)=\tilde{\sigma}^{r r}\left(R_{2}^{+}\right)$, and $\tilde{r}\left(R_{2}^{-}\right)=\tilde{r}\left(R_{2}^{+}\right)$.

For $R_{1} \leq R \leq R_{2}, \mathbf{G}=\operatorname{diag}\left\{1, R^{2}\right\}$ and $\mathbf{g}=\operatorname{diag}\left\{1, \tilde{r}^{2}(R)\right\}$. Therefore, incompressibility gives $\tilde{r}^{2}(R)=$ $R^{2}+\tilde{r}_{2}^{2}-R_{2}^{2}$, with $\tilde{r}_{2}$ to be calculated. The Cauchy stress has the form given in (3.15) with $\tilde{r}$ instead of $r$. Therefore, the radial component of the Cauchy stress reads (note that $p_{i}=0$ )

$$
\tilde{\sigma}^{r r}(R)=\int_{R_{1}}^{R} \frac{\alpha(x)+\beta(x)}{x}\left[1-\frac{x^{4}}{\tilde{r}^{4}(x)}\right] d x .
$$

In particular

$$
\tilde{\sigma}^{r r}\left(R_{2}^{-}\right)=\int_{R_{1}}^{R_{2}} \frac{\alpha(x)+\beta(x)}{x}\left[1-\frac{x^{4}}{\tilde{r}^{4}(x)}\right] d x .
$$

For $R_{2} \leq R \leq R_{2}+\Delta^{\prime}, \mathbf{G}=\operatorname{diag}\left\{{r^{\prime}}^{2}(R), r^{2}(R)\right\}$ and $\mathbf{g}=\operatorname{diag}\left\{1, \tilde{r}^{2}\right\}$. Therefore, incompressibility implies that $\tilde{r}^{2}(R)=r^{2}(R)-r_{2}^{2}+\tilde{r}_{2}^{2}$. The principal invariants of $\tilde{\mathbf{b}}$ in this region read

$$
\tilde{I}_{1}=\frac{\tilde{r}^{2}(R)}{r^{2}(R)}+\frac{r^{2}(R)}{\tilde{r}^{2}(R)}, \quad \tilde{I}_{2}=1
$$

Hence, the Cauchy stress in this region has the following representation

$$
\tilde{\boldsymbol{\sigma}}(R)=\left(\begin{array}{cc}
-p(R)+\frac{r^{2}(R)}{\tilde{r}^{2}(R)} \alpha(R)+\left(1-\frac{\tilde{r}^{2}(R)}{r^{2}(R)}\right) \beta(R) & 0 \\
0 & -\frac{p(R)}{\tilde{r}^{2}(R)}+\frac{\alpha(R)}{r^{2}(R)}+\left(1-\frac{r^{2}(R)}{\tilde{r}^{2}(R)}\right) \frac{\beta(R)}{\tilde{r}^{2}(R)}
\end{array}\right) .
$$


Using the radial equilibrium equation, one obtains

$$
\begin{aligned}
\tilde{\sigma}^{r r}(R) & =\tilde{\sigma}^{r r}\left(R_{2}^{-}\right)+\int_{R_{2}}^{R} \frac{\alpha(x)+\beta(x)}{r(x)}\left[1-\frac{r^{4}(x)}{\tilde{r}^{4}(x)}\right] r^{\prime}(x) d x \\
& =\tilde{\sigma}_{2}+\int_{r_{2}}^{r(R)} \frac{\alpha(\xi)+\beta(\xi)}{\xi}\left[1-\frac{\xi^{4}}{\left(\xi^{2}-r_{2}^{2}+\tilde{r}_{2}^{2}\right)^{2}}\right] d \xi
\end{aligned}
$$

where $\tilde{\sigma}_{2}=\tilde{\sigma}^{r r}\left(R_{2}^{-}\right)$. We know that $\tilde{\sigma}^{r r}\left(R_{2}+\Delta^{\prime}\right)=0$ and hence

$$
\int_{R_{1}}^{R_{2}} \frac{\alpha(x)+\beta(x)}{x}\left[1-\frac{x^{4}}{\left(x^{2}-R_{2}^{2}+\tilde{r}_{2}^{2}\right)^{2}}\right] d x+\int_{r_{2}}^{r\left(R_{2}+\Delta^{\prime}\right)} \frac{\alpha(\xi)+\beta(\xi)}{\xi}\left[1-\frac{\xi^{4}}{\left(\xi^{2}-r_{2}^{2}+\tilde{r}_{2}^{2}\right)^{2}}\right] d \xi=0,
$$

which is the equation that determines the unknown constant $\tilde{r}_{2}$.

Remark 3.1. Note that the residual stress distribution is independent of the choice of $\psi^{+}(R, \Theta)=(r(R), \Theta)$. One should keep in mind that to be more precise the material manifold for $r_{2} \leq r \leq r_{2}+\Delta$ is identified by the inverse of the map $r(R)$. Hence, choosing another map $\hat{r}$ the corresponding ring in the material manifold has radius $\hat{R}=\hat{r}^{-1}(r)$. For this choice, Eq. (3.27) becomes

$$
\tilde{\sigma}^{r r}(\hat{R})=\tilde{\sigma}_{2}+\int_{r_{2}}^{\hat{r}(\hat{R})} \frac{\alpha(\xi)+\beta(\xi)}{\xi}\left[1-\frac{\xi^{4}}{\left(\xi^{2}-r_{2}^{2}+\tilde{r}_{2}^{2}\right)^{2}}\right] d \xi
$$

Noting that $\hat{r}(\hat{R})=r(R)$ one observes that $\tilde{\sigma}^{r r}(\hat{R})=\tilde{\sigma}^{r r}(R)$, as expected.

Remark 3.2. If the added material is different from the material of the initial body (note that energy functions are defined with respect to their stress-free configurations) the calculations do not change much. Suppose the initial body has an energy function $W^{(i)}=W^{(i)}\left(I_{1}, I_{2}\right)$ and the accreted body has the energy function $W^{(i i)}=W^{(i i)}\left(I_{1}, I_{2}\right)$. The material metric would not be affected. However, in this case $\alpha$ and $\beta$ functions are defined as

$$
\begin{array}{r}
R_{1} \leq R \leq R_{2}: \quad \alpha=2 \frac{\partial W^{(i)}}{\partial I_{1}}, \beta=2 \frac{\partial W^{(i)}}{\partial I_{2}} \\
R_{2} \leq R \leq R_{2}+\Delta: \quad \alpha=2 \frac{\partial W^{(i i)}}{\partial I_{1}}, \beta=2 \frac{\partial W^{(i i)}}{\partial I_{2}} .
\end{array}
$$

\section{Continuous Surface Growth of an Infinitely Long Thick Hollow Cylinder}

In this section we formulate the initial-boundary value problem of symmetric accretion of a thick hollow cylinder made of an incompressible isotropic hyperelastic solid. We consider two cases: outer and inner accretions. In the outer accretion problem stress-free (or pre-stressed) material is continuously added to the traction-free outer boundary of the deformed cylinder. In the inner accretion problem, material is continuously added to the inner boundary of the deformed body. We will see that the governing equations include a nonlinear partial integral equation that will be solved numerically for a neo-Hookean solid. We will linearize the governing equations of the nonlinear surface growth and will show that the resulting stresses are identical to those calculated using the linear theory of Brown and Goodman [1963].

\subsection{Outer Accretion}

Let us assume that the initial stress-free body is an infinitely-long solid cylinder with inner radius $R_{1}$ and outer radius $R_{0}$. At time $t$, under an internal pressure $p_{i}(t)$, the outer radius in the current configuration is $r_{2}(t)$. We assume that accretion velocity is normal to the boundary with magnitude $u_{g}(t)$. This means that in the infinitesimal time interval $[t, t+d t]$ a stress-free ring of thickness $u_{g}(t) d t$ is added to the deformed body. The metric of the Euclidean ambient space in polar coordinates $(r, \theta)$ reads

$$
\mathbf{g}=\left(\begin{array}{cc}
1 & 0 \\
0 & r^{2}
\end{array}\right)
$$

In the material manifold $\mathcal{B}$ we use the polar coordinates $(R, \Theta)$. 


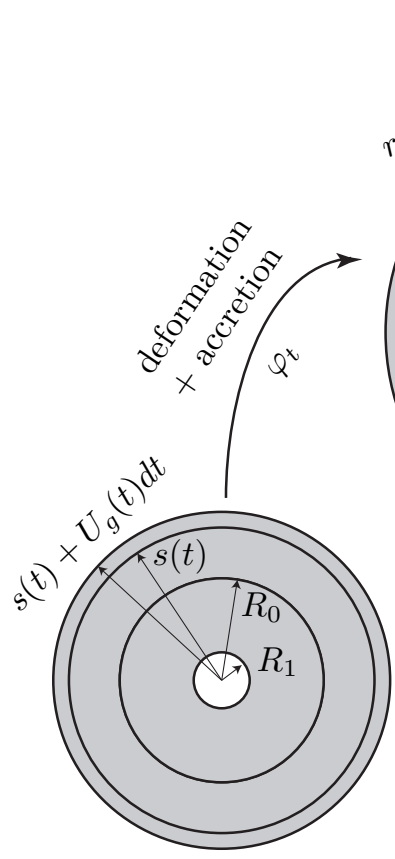

(a)

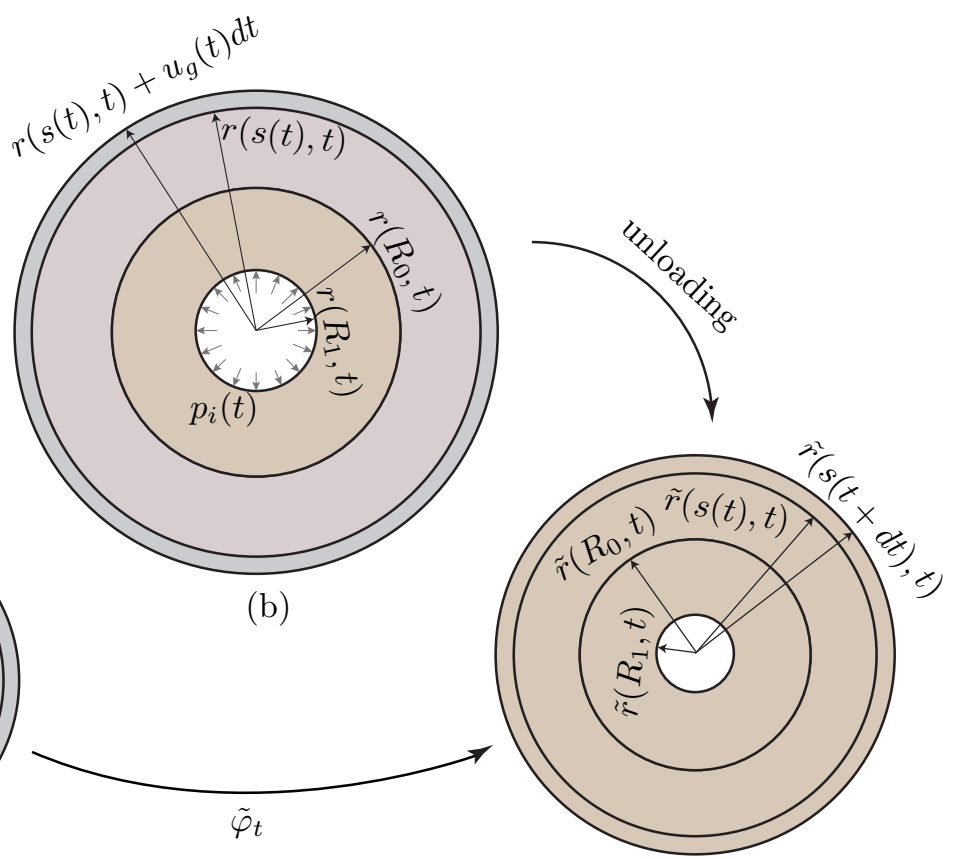

(c)

Figure 3: Continuous Outer Accretion: (a) The material manifold $(\mathcal{B}, \mathbf{G})$. The outer radius at time $t$ is $s(t)$. At a later time $t+d t$ the outer radius is changed to $s(t)+U_{g}(t) d t$. (b) The deformed annulus under a specified internal pressure $p_{i}(t)$ with a layer of stress-feee material of thickness $u_{g}(t) d t$ attached to its outer boundary during the time interval $[t, t+d t]$. (c) The residually-stressed accreted body after removal of external forces (here the internal pressure $p_{i}(t)$ ).

\subsubsection{Kinematics and material metric}

We assume motions of the form $(r, \theta)=(r(R, t), \Theta)$, and hence the deformation gradient reads

$$
\mathbf{F}=\left(\begin{array}{cc}
r^{\prime}(R, t) & 0 \\
0 & 1
\end{array}\right)
$$

where $r^{\prime}(R, t)=\frac{\partial r(R, t)}{\partial R}$. We assign to each layer at radius $R$ in the material manifold a time of accretion $\tau(R)$. This map is assumed to be invertible, meaning that there is no ablation during the surface growth process. We indicate its inverse with $s=\tau^{-1}$, which assigns to each time the radius of the accreted ring. We assume that $\tau\left(R_{0}\right)=0$. In this problem the growth surfaces in the material manifold and the current configuration are defined as

$$
\Omega_{t}=\{(s(t), \Theta): 0 \leq \Theta<2 \pi\}, \quad \omega_{t}=\{(r(s(t), t), \theta): 0 \leq \theta<2 \pi\} .
$$

Note that

$$
\frac{d}{d t} r(s(t), t)=\frac{\partial r}{\partial R}(s(t), t) \dot{s}(t)+\frac{\partial r}{\partial t}(s(t), t)=r^{\prime}(s(t), t) U_{g}(t)+V^{r}(s(t), t),
$$

where $V^{r}=\frac{\partial r}{\partial t}$ is the radial component of the material velocity on the growth surface and $U_{g}(t)=\dot{s}(t)$. We observe that in the absence of accretion, the spatial velocity of the material points lying on the outer boundary is simply $V^{r}(s(t), t)$. This means that

$$
u_{g}(t)=r^{\prime}(s(t), t) U_{g}(t)
$$

Note that we are assuming that growth velocity is normal to the boundary both in the reference and the current configurations. For $t=\tau(R),(4.5)$ can be rewritten as

$$
r^{\prime}(R, \tau(R))=\tau^{\prime}(R) u_{g}(\tau(R)) .
$$

Similar to our discussion of the discrete accretion problem, for the sake of simplicity of calculations, we assume that in the reference configuration in the time interval $[t, t+d t]$ the set $s(t)<R \leq s(t)+u_{g}(t) d t$ is added to the material manifold. This choice corresponds to setting $U_{g}(t)=u_{g}(t)$. Therefore, from (4.5) we conclude that

$$
r^{\prime}(s(t), t)=1 \quad \text { or } \quad r^{\prime}(R, \tau(R))=1 .
$$


Also, the choice $U_{g}(t)=u_{g}(t)$ implies that

$$
s(t)=\int_{0}^{t} u_{g}(\eta) d \eta+R_{0}
$$

To simplify the calculations, we assume that the spatial growth velocity is constant, i.e. $u_{g}(t)=u_{0}>0$. Hence

$$
s(t)=R_{0}+u_{0} t, \text { or } \quad \tau(R)=\frac{R-R_{0}}{u_{0}} .
$$

In this case, (4.7) reads

$$
r^{\prime}\left(R_{0}+u_{0} t, t\right)=1 \quad \text { or } \quad r^{\prime}\left(R, \frac{R-R_{0}}{u_{0}}\right)=1 .
$$

Note that any non-negative function of $t$ whose set of zeros has no limit points would be an acceptable choice for $U_{g}(t)$ and will have a corresponding consistent material metric.

For $R_{1} \leq R \leq R_{0}$, we know that the material metric has the following representation in polar coordinates.

$$
\mathbf{G}=\left(\begin{array}{cc}
1 & 0 \\
0 & R^{2}
\end{array}\right)
$$

For $R_{0} \leq R \leq s(t)$, the accreted ring at any instant of time $t$ is stress-free and hence the material metric at $R=s(t)$ is the pull back of the metric of the Euclidean ambient space metric, i.e.

$$
\mathbf{G}(s(t))=\varphi_{t}^{*} \mathbf{g}(r(s(t), t)) \text {, or equivalently } \quad \mathbf{G}(R)=\varphi_{\tau(R)}^{*} \mathbf{g}(r(R, \tau(R))) .
$$

In components, this reads (c.f. $(2.8))$

$$
G_{A B}(s(t))=G_{A B}(R)=F_{A}^{a}(R, \tau(R)) F_{B}^{b}(R, \tau(R)) g_{a b}(r(R, \tau(R))) .
$$

Thus, we have

$$
\mathbf{G}=\left(\begin{array}{cc}
r^{2}(R, \tau(R)) & 0 \\
0 & r^{2}(R, \tau(R))
\end{array}\right)=\left(\begin{array}{cc}
1 & 0 \\
0 & r^{2}(R, \tau(R))
\end{array}\right)=\left(\begin{array}{cc}
1 & 0 \\
0 & r^{2}\left(R, \frac{R-R_{0}}{u_{0}}\right)
\end{array}\right)
$$

where we have used (4.9) and (4.10). Therefore, the material manifold is an evolving Riemannian manifold $\left(\mathcal{B}_{t}, \mathbf{G}\right)$, where

$$
\mathcal{B}_{t}=\left\{(R, \Theta): 0 \leq \Theta<2 \pi, R_{1} \leq R \leq s(t)=R_{0}+u_{0} t\right\},
$$

and

$$
\begin{aligned}
R_{1} \leq R \leq R_{0}: \quad \mathbf{G}=\left(\begin{array}{cc}
1 & 0 \\
0 & R^{2}
\end{array}\right) \\
R_{0} \leq R_{0}+u_{0} t: \quad \mathbf{G}=\left(\begin{array}{cc}
1 & 0 \\
0 & r^{2}\left(R, \frac{R-R_{0}}{u_{0}}\right)
\end{array}\right) .
\end{aligned}
$$

The incompressibility condition reads

$$
J=\sqrt{\frac{\operatorname{det} \mathbf{g}}{\operatorname{det} \mathbf{G}}} \operatorname{det} \mathbf{F}=1 .
$$

For $R_{1} \leq R \leq R_{0}$ this implies that $r(R, t) r^{\prime}(R, t)=R$, and therefore

$$
r^{2}(R, t)=r_{0}^{2}(t)+R^{2}-R_{0}^{2}, \quad R_{1} \leq R \leq R_{0},
$$

where $r_{0}(t):=r\left(R_{0}, t\right)$. For $R_{0} \leq R \leq R_{0}+u_{0} t$, incompressibility implies that

$$
r(R, t) r^{\prime}(R, t)=r\left(R, \frac{R-R_{0}}{u_{0}}\right)
$$


which can be integrated for example between $R_{0}$ and $R$ to give

$$
r^{2}(R, t)=r^{2}\left(R_{0}, t\right)+\int_{R_{0}}^{R} 2 r\left(x, \frac{x-R_{0}}{u_{0}}\right) d x, \quad R_{0} \leq R \leq R_{0}+u_{0} t .
$$

Note that the first term on the right-hand side of the above equation only depends on $t$, while the second term depends only on $R$, i.e. incompressibility dictates that $r^{2}(R, t)$ has an additive decomposition into an $R$-dependent and a $t$-dependent function. Defining $\bar{r}(R):=r\left(R, \frac{R-R_{0}}{u_{0}}\right)$ we can write

$$
r^{2}(R, t)=r_{0}^{2}(t)+\int_{R_{0}}^{R} 2 \bar{r}(x) d x, \quad R_{0} \leq R \leq R_{0}+u_{0} t .
$$

Remark 4.1. In the case of constant growth velocity $u_{g}(t)=u_{0}$, as we mentioned earlier $U_{g}(t)=u_{0}$ is not the only choice. One can choose, for example, $U_{g}(t)=U_{0}$ for any constant $U_{0}\left(U_{0}>0\right)$. Note that the material manifold explicitly depends on the constant $U_{0}$. In this case, the material manifold and metric have the following representations

$$
\mathcal{B}_{t}=\left\{(R, \Theta): 0 \leq \Theta<2 \pi, R_{1} \leq R \leq s(t)=R_{0}+U_{0} t\right\}
$$

and

$$
\begin{aligned}
R_{1} \leq R \leq R_{0}: \quad \mathbf{G}=\left(\begin{array}{cc}
1 & 0 \\
0 & R^{2}
\end{array}\right) \\
R_{0} \leq R \leq R_{0}+U_{0} t: \quad \mathbf{G}=\left(\begin{array}{cc}
\left(\frac{u_{0}}{U_{0}}\right)^{2} & 0 \\
0 & r^{2}\left(R, \frac{R-R_{0}}{U_{0}}\right)
\end{array}\right) .
\end{aligned}
$$

Note that, similarly to what was shown in the case of discrete accretion, the accretion stresses will be independent of this choice. In other words, the material manifold is not unique. However, the material manifolds given by Eqs. (4.22) and (4.23) are equivalent in the sense that using any of them the same stresses are calculated. This will be shown in Remark 4.3.

\subsubsection{Calculation of stresses for an incompressible isotropic accreting cylinder}

We next calculate the stress field during the accretion process. For $R_{1} \leq R \leq R_{0}$, Finger tensor $\mathbf{b}^{\sharp}$ and $\mathbf{b}^{-1}$ have the same forms as given in (3.12) and (3.14), while principal stretches have forms identical to those in (3.13). Using equilibrium equations, the radial Cauchy stress has the following expression

$$
\sigma^{r r}(R, t)=-p_{i}(t)+\int_{R_{1}}^{R} \frac{\alpha(x, t)+\beta(x, t)}{x}\left[1-\frac{x^{4}}{r^{4}(x, t)}\right] d x .
$$

For $R_{0} \leq R \leq R_{0}+u_{0} t$, the Finger tensor $\mathbf{b}^{\sharp}$, and $\mathbf{b}^{-1}$ read

$$
\mathbf{b}^{\sharp}=\left(\begin{array}{cc}
\frac{\bar{r}^{2}(R)}{r^{2}(R, t)} & 0 \\
0 & \frac{1}{\bar{r}^{2}(R)}
\end{array}\right), \quad \mathbf{b}^{-1}=\left(\begin{array}{cc}
\frac{r^{2}(R, t)}{\bar{r}^{2}(R)} & 0 \\
0 & \frac{\bar{r}^{2}(R)}{r^{4}(R, t)}
\end{array}\right) .
$$

The principal invariants of $\mathbf{b}$ read

$$
I_{1}=\frac{r^{2}(R, t)}{\bar{r}^{2}(R)}+\frac{\bar{r}^{2}(R)}{r^{2}(R, t)}, \quad I_{2}=1 .
$$

The Cauchy stress in this region has the following representation

$$
\boldsymbol{\sigma}=\left(\begin{array}{cc}
-p(R, t)+\frac{\bar{r}^{2}(R)}{r^{2}(R, t)} \alpha(R, t)+\left(1-\frac{r^{2}(R, t)}{\bar{r}^{2}(R)}\right) \beta(R, t) & 0 \\
0 & -\frac{p(R, t)}{r^{2}(R, t)}+\frac{\alpha(R, t)}{\bar{r}^{2}(R)}+\left(1-\frac{\bar{r}^{2}(R)}{r^{2}(R, t)}\right) \frac{\beta(R, t)}{r^{2}(R, t)}
\end{array}\right) .
$$


Using the radial equilibrium equation, the radial Cauchy stress is calculated as

$$
\begin{aligned}
\sigma^{r r}(R, t) & =\sigma^{r r}\left(R_{0}, t\right)+\int_{R_{0}}^{R} \frac{\alpha(x, t)+\beta(x, t)}{\bar{r}(x)}\left[1-\frac{\bar{r}^{4}(x)}{r^{4}(x, t)}\right] d x \\
& =-p_{i}(t)+\int_{R_{1}}^{R_{0}} \frac{\alpha(x, t)+\beta(x, t)}{x}\left[1-\frac{x^{4}}{r^{4}(x, t)}\right] d x+\int_{R_{0}}^{R} \frac{\alpha(x, t)+\beta(x, t)}{\bar{r}(x)}\left[1-\frac{\bar{r}^{4}(x)}{r^{4}(x, t)}\right] d x .
\end{aligned}
$$

We know that $\sigma^{r r}(s(t), t)=\sigma^{\theta \theta}(s(t), t)=0 .{ }^{6}$ In particular, for the radial component this gives us

$$
\int_{R_{1}}^{R_{0}} \frac{\alpha(x, t)+\beta(x, t)}{x}\left[1-\frac{x^{4}}{r^{4}(x, t)}\right] d x+\int_{R_{0}}^{R_{0}+u_{0} t} \frac{\alpha(x, t)+\beta(x, t)}{\bar{r}(x)}\left[1-\frac{\bar{r}^{4}(x)}{r^{4}(x, t)}\right] d x=p_{i}(t) .
$$

Here the unknowns are $r_{0}(t)$ and $\bar{r}(R)$ with the initial conditions $r_{0}(0)=\bar{r}\left(R_{0}\right)=R_{0}$. Note that $\left(\bar{\sigma}^{\theta \theta}\right.$ is the physical component corresponding to $\sigma^{\theta \theta}$.)

$$
\bar{\sigma}^{\theta \theta}(R, t)=r^{2}(R, t) \sigma^{\theta \theta}(R, t)=\sigma^{r r}(R, t)+\left[\frac{r^{2}(R, t)}{\bar{r}^{2}(R)}-\frac{\bar{r}^{2}(R)}{r^{2}(R, t)}\right][\alpha(R, t)+\beta(R, t)] .
$$

Therefore

$$
\bar{\sigma}^{\theta \theta}(s(t), t)=\sigma^{r r}(s(t), t)+\left[\frac{r^{2}(s(t), t)}{\bar{r}^{2}(s(t))}-\frac{\bar{r}^{2}(s(t))}{r^{2}(s(t), t)}\right][\alpha(s(t), t)+\beta(s(t), t)]=0,
$$

as by definition $\bar{r}(s(t))=r(s(t), t)$, i.e. the above condition is trivially satisfied.

In the case of a homogeneous neo-Hookean solid, $\alpha(R, t)=\mu$ and $\beta(R, t)=0$. In this case, Eq. (4.29) is simplified to read

$$
\int_{R_{1}}^{R_{0}} \frac{1}{x}\left[1-\frac{x^{4}}{r^{4}(x, t)}\right] d x+\int_{R_{0}}^{R_{0}+u_{0} t} \frac{1}{\bar{r}(x)}\left[1-\frac{\bar{r}^{4}(x)}{r^{4}(x, t)}\right] d x=\frac{p_{i}(t)}{\mu} .
$$

In summary, we have the following problem in the triangular region $\left\{(R, t) \in \mathbb{R}^{2} \mid R_{0} \leq R \leq R_{0}+u_{0} t, t \in \mathbb{R}^{+}\right\}$:

$$
\left\{\begin{array}{l}
r(R, t) r^{\prime}(R, t)=r\left(R, \frac{R-R_{0}}{u_{0}}\right), \\
\int_{R_{1}}^{R_{0}} \frac{1}{x}\left[1-\frac{x^{4}}{\left(r^{2}\left(R_{0}, t\right)+x^{2}-R_{0}^{2}\right)^{2}}\right] d x+\int_{R_{0}}^{R_{0}+u_{0} t} \frac{1}{\bar{r}(x)}\left[1-\frac{\bar{r}^{4}(x)}{r^{4}(x, t)}\right] d x=\frac{p_{i}(t)}{\mu}, \\
r\left(R_{0}, 0\right)=R_{0},
\end{array}\right.
$$

where the first equation is a partial differential equation, the second is a nonlinear integral equation, and the third is the initial condition. Note that the condition $r\left(R_{0}, 0\right)=R_{0}$ implies that for $R_{1} \leq R \leq R_{0}, r(R, 0)=R$. Furthermore, we assume that the internal pressure satisfies $p_{i}(0)=0$.

Remark 4.2. Suppose that the accreted body is inhomogeneous and is made of a material possibly different from that of the initial body. This would not affect the material metric. However, for the accreted body $\left(R_{0} \leq R \leq s(t)\right)$, the energy function is inhomogeneous, i.e. $W=W\left(R, I_{1}, I_{2}\right)=\hat{W}\left(\tau(R), I_{1}, I_{2}\right)$. This means that the functions $\alpha$ and $\beta$ in the interval $R_{0} \leq R \leq s(t)$ should be modified as follows

$$
\alpha(R, t)=2 \frac{\partial W\left(R, I_{1}, I_{2}\right)}{\partial I_{1}}, \quad \beta=2 \frac{\partial W\left(R, I_{1}, I_{2}\right)}{\partial I_{2}} .
$$

Remark 4.3. We now prove that the stress distribution is independent of the choice of $U_{0}>0$ in (4.23). We denote the radial coordinate in the new material manifold by $\check{R}$. For $R_{1} \leq R \leq R_{0}, \check{R}=R$. We know that in the two material manifolds the time of attachment of a layer of stress-free material should be the same,

\footnotetext{
${ }^{6}$ It is assumed that the material added to the boundary in the current configuration is stress-free and hence at $r_{2}(t)$, stress tensor, and not just the traction vector, vanishes.
} 
i.e. $\check{\tau}(\check{R})=\tau(R)$. Note that $R=R_{0}+u_{0} \tau(R)$. Similarly, $\check{R}=R_{0}+U_{0} \check{\tau}(\check{R})=R_{0}+U_{0} \tau(R)$. Thus, for $R_{0} \leq R \leq R_{0}+u_{0} t$

$$
\check{R}(R)=R_{0}\left(1-\frac{U_{0}}{u_{0}}\right)+\frac{U_{0}}{u_{0}} R
$$

Let us denote the radial component of the deformation mapping with respect to the new material manifold by $\check{r}(\check{R}, t)$. We also know that $r(R, t)=\check{r}(\check{R}, t)$. Thus

$$
\bar{r}(\check{R})=\check{r}(\check{R}, \check{\tau}(\check{R}))=\check{r}(\check{R}, \tau(R))=r(R, \tau(R))=\bar{r}(R) .
$$

It is straightforward to show that with respect to the new material manifold (4.23), $\mathbf{b}^{\sharp}, \mathbf{b}^{-1}, I_{1}$, and $I_{2}$ will remain unchanged and hence the stress distribution will not change. In other words, the Cauchy stress is independent of the choice $U_{0}>0$.

Remark 4.4. Suppose the accreted ring (cylinder) is not stress-free. This means that if the ring added at time $\tau(R)$ is allowed to relax, its radius in the relaxed configuration will be $\chi(R) \neq \bar{r}(R)$. We still choose $U_{g}=u_{g}$, i.e., (4.10) is still valid. What changes now is that the material manifold inherits its metric from the relaxed (or natural) configuration, and not from the deformed configuration. Denoting by $d \Delta$ the thickness of this ring in its local relaxed configuration, we can express incompressibility as

$$
2 \pi \chi(R) d \Delta=2 \pi \bar{r}(R) u_{0} d t,
$$

where $u_{0} d t$ is the thickness of the added material in the deformed configuration. Thus, $d \Delta / u_{0} d t=\bar{r}(R) / \chi(R)$. In the material manifold the thickness is $u_{0} d t$ as well because of our choice $U_{g}(t)=u_{g}(t)$. Therefore, the local deformation gradient from the material manifold to the local relaxed configuration is

$$
\mathbf{F}_{\chi}(R)=\left(\begin{array}{cc}
\frac{\bar{r}(R)}{\chi(R)} & 0 \\
0 & 1
\end{array}\right)
$$

We also know that in the local relaxed configuration the metric $\mathbf{g}_{\chi}$ is Euclidean, and it is written as

$$
\mathbf{g}_{\chi}(R)=\left(\begin{array}{cc}
1 & 0 \\
0 & \chi^{2}(R)
\end{array}\right)
$$

Now the material metric is $\mathbf{G}=\chi^{*} \mathbf{g}_{\chi}$, i.e.

$$
\mathbf{G}(R)=\left(\begin{array}{cc}
\bar{r}^{2}(R) & 0 \\
\chi^{2}(R) & \chi^{2}(R)
\end{array}\right)
$$

Note that for $\chi(R)=\bar{r}(R)$ we recover the stress-free case. Note also that $\operatorname{det} \mathbf{G}=\bar{r}^{2}(R)$ is independent of $\chi(R)$, and hence the incompressibility equation remains. The kinematics and stress analysis in the initial body $\left(R_{1} \leq R \leq R_{0}\right)$ remains unchanged. For $R_{0} \leq R \leq R_{0}+u_{0} t$, the Finger tensor $\mathbf{b}^{\sharp}$, and $\mathbf{b}^{-1}$ read

$$
\mathbf{b}^{\sharp}=\left(\begin{array}{cc}
\frac{\chi^{2}(R)}{r^{2}(R, t)} & 0 \\
0 & \frac{1}{\chi^{2}(R)}
\end{array}\right), \quad \mathbf{b}^{-1}=\left(\begin{array}{cc}
\frac{r^{2}(R, t)}{\chi^{2}(R)} & 0 \\
0 & \frac{\chi^{2}(R)}{r^{4}(R, t)}
\end{array}\right) .
$$

The principal invariants of $\mathbf{b}$ read

$$
I_{1}=\frac{r^{2}(R, t)}{\chi^{2}(R)}+\frac{\chi^{2}(R)}{r^{2}(R, t)}, \quad I_{2}=1 .
$$

The Cauchy stress would have the following representation

$$
\boldsymbol{\sigma}=\left(\begin{array}{cc}
-p(R, t)+\frac{\chi^{2}(R)}{r^{2}(R, t)} \alpha(R, t)+\left(1-\frac{r^{2}(R, t)}{\chi^{2}(R)}\right) \beta(R, t) & 0 \\
0 & -\frac{p(R, t)}{r^{2}(R, t)}+\frac{\alpha(R, t)}{\chi^{2}(R)}+\left(1-\frac{\chi^{2}(R)}{r^{2}(R, t)}\right) \frac{\beta(R, t)}{r^{2}(R, t)}
\end{array}\right) .
$$


The radial Cauchy stress is written as

$$
\begin{aligned}
\sigma^{r r}(R, t)= & \sigma^{r r}\left(R_{0}, t\right)+\int_{R_{0}}^{R} \frac{\alpha(x, t)+\beta(x, t)}{\chi^{2}(x)} \bar{r}(x)\left[1-\frac{\chi^{4}(x)}{r^{4}(x, t)}\right] d x \\
= & -p_{i}(t)+\int_{R_{1}}^{R_{0}} \frac{\alpha(x, t)+\beta(x, t)}{x}\left[1-\frac{x^{4}}{r^{4}(x, t)}\right] d x \\
& +\int_{R_{0}}^{R} \frac{\alpha(x, t)+\beta(x, t)}{\chi^{2}(x)} \bar{r}(x)\left[1-\frac{\chi^{4}(x)}{r^{4}(x, t)}\right] d x
\end{aligned}
$$

If we assume that the outer boundary is traction-free at all times we have the boundary condition $\sigma^{r r}(s(t), t)=0$. Therefore

$$
\int_{R_{1}}^{R_{0}} \frac{\alpha(x, t)+\beta(x, t)}{x}\left[1-\frac{x^{4}}{r^{4}(x, t)}\right] d x+\int_{R_{0}}^{R} \frac{\alpha(x, t)+\beta(x, t)}{\chi^{2}(x)} \bar{r}(x)\left[1-\frac{\chi^{4}(x)}{r^{4}(x, t)}\right] d x=p_{i}(t) .
$$

When the added layer is pre-stressed it can be shown that

$$
\bar{\sigma}^{\theta \theta}(R, t)=\sigma^{r r}(R, t)+\left[\frac{r^{2}(R, t)}{\chi^{2}(R)}-\frac{\chi^{2}(R)}{r^{2}(R, t)}\right][\alpha(R, t)+\beta(R, t)] .
$$

Thus

$$
\bar{\sigma}^{\theta \theta}(s(t), t)=\left[\frac{\bar{r}^{2}(s(t))}{\chi^{2}(s(t))}-\frac{\chi^{2}(s(t))}{\bar{r}^{2}(s(t))}\right][\alpha(s(t), t)+\beta(s(t), t)] .
$$

It is seen that unlike the previous case, $\bar{\sigma}^{\theta \theta}(s(t), t) \neq 0$, in general.

\subsubsection{Calculation of residual stresses for an incompressible isotropic accreting cylinder}

Suppose at some time $t=t_{a}$, the accretion process stops. For any $t>t_{a}$, if one removes the internal pressure the accreted body would be residually-stressed. The residual stress distribution depends on the accretion characteristics (here the constant growth velocity $u_{0}$ ) and the history of the applied loads in the time interval $t \in$ $\left[0, t_{a}\right]$, i.e., the history of the internal pressure $\left\{p_{i}(t), t \in\left[0, t_{a}\right]\right\}$. Material metric of the additively manufactured cylinder has the following representation:

$$
\begin{aligned}
& R_{1} \leq R \leq R_{0}: \quad \mathbf{G}=\left(\begin{array}{cc}
1 & 0 \\
0 & R^{2}
\end{array}\right) \\
& R_{0} \leq R \leq R_{a}: \quad \mathbf{G}=\left(\begin{array}{cc}
1 & 0 \\
0 & \bar{r}^{2}(R)
\end{array}\right)
\end{aligned}
$$

Note that the function $r(R, t)$ and hence $\bar{r}(R)=r\left(R, \frac{R-R_{0}}{u_{0}}\right)$ have already been calculated. The motion from the material manifold to the residually-stressed configuration (under no applied loads) is denoted by $\tilde{\varphi}: \mathcal{B} \rightarrow \mathcal{S}$, where in polar coordinates we have: $\tilde{\varphi}(R, \Theta)=(\tilde{r}, \tilde{\theta})=(\tilde{r}(R), \Theta)$, see Fig.3. Using the incompressibility constraint one obtains

$$
\begin{array}{ll}
\tilde{r}^{2}(R)=\tilde{r}^{2}\left(R_{0}\right)+R^{2}-R_{0}^{2}, & R_{1} \leq R \leq R_{0}, \\
\tilde{r}^{2}(R)=\tilde{r}^{2}\left(R_{0}\right)+\int_{R_{0}}^{R} 2 \bar{r}^{2}(x) d x, & R_{0} \leq R \leq R_{a},
\end{array}
$$

where $\tilde{r}\left(R_{0}\right)$ is an unknown that will be determined after enforcing the boundary and continuity conditions. For the deformation mapping $\tilde{\varphi}$ we have

$$
\begin{array}{ll}
I_{1}=\frac{\tilde{r}^{2}(R)}{R^{2}}+\frac{R^{2}}{\tilde{r}^{2}(R)}, \quad I_{2}=1, \quad R_{1} \leq R \leq R_{0}, \\
I_{1}=\frac{\tilde{r}^{2}(R)}{\bar{r}^{2}(R)}+\frac{\bar{r}^{2}(R)}{\tilde{r}^{2}(R)}, \quad I_{2}=1, \quad R_{0} \leq R \leq R_{a} .
\end{array}
$$


In the absence of applied loads the boundary conditions read: $\tilde{\sigma}^{r r}\left(R_{1}\right)=\tilde{\sigma}^{r r}\left(R_{a}\right)=0$. One then has the following distribution of radial Cauchy stress (the continuity of traction $\tilde{\sigma}^{r r}\left(R_{0}^{-}\right)=\tilde{\sigma}^{r r}\left(R_{0}^{+}\right)$has been enforced):

$$
\begin{aligned}
& R_{1} \leq R \leq R_{0}: \quad \tilde{\sigma}^{r r}(R)=\int_{R_{1}}^{R} \frac{\tilde{\alpha}(x)+\tilde{\beta}(x)}{x}\left[1-\frac{x^{4}}{\tilde{r}^{4}(x)}\right] d x \\
& R_{0} \leq R \leq R_{a}: \quad \tilde{\sigma}^{r r}(R)=\int_{R_{1}}^{R_{0}} \frac{\tilde{\alpha}(x)+\tilde{\beta}(x)}{x}\left[1-\frac{x^{4}}{\tilde{r}^{4}(x)}\right] d x+\int_{R_{0}}^{R} \frac{\tilde{\alpha}(x)+\tilde{\beta}(x)}{\bar{r}(x)}\left[1-\frac{\bar{r}^{4}(x)}{\tilde{r}^{4}(x)}\right] d x .
\end{aligned}
$$

Similarly, for the physical circumferential Cauchy stress component we have

$$
\begin{aligned}
& R_{1} \leq R \leq R_{0}: \quad \overline{\tilde{\sigma}}^{\theta \theta}(R)=\tilde{\sigma}^{r r}(R)+[\tilde{\alpha}(R)+\tilde{\beta}(R)]\left[\frac{\tilde{r}^{2}(R)}{R^{2}}-\frac{R^{2}}{\tilde{r}^{2}(R)}\right], \\
& R_{0} \leq R \leq R_{a}: \quad \overline{\tilde{\sigma}}^{\theta \theta}(R)=\tilde{\sigma}^{r r}(R)+[\tilde{\alpha}(R)+\tilde{\beta}(R)]\left[\frac{\tilde{r}^{2}(R)}{\bar{r}^{2}(R)}-\frac{\bar{r}^{2}(R)}{\tilde{r}^{2}(R)}\right] .
\end{aligned}
$$

Note that, in general, $\tilde{r}\left(R_{a}\right) \neq \bar{r}\left(R_{a}\right)$, and hence $\overline{\tilde{\sigma}}^{\theta \theta}\left(R_{a}\right) \neq 0$. The unknown constant $\tilde{r}\left(R_{0}\right)$ is determined using the following condition

$$
\tilde{\sigma}^{r r}\left(R_{a}\right)=\int_{R_{1}}^{R_{0}} \frac{\tilde{\alpha}(x)+\tilde{\beta}(x)}{x}\left[1-\frac{x^{4}}{\tilde{r}^{4}(x)}\right] d x+\int_{R_{0}}^{R_{a}} \frac{\tilde{\alpha}(x)+\tilde{\beta}(x)}{\bar{r}(x)}\left[1-\frac{\bar{r}^{4}(x)}{\tilde{r}^{4}(x)}\right] d x=0 .
$$

Remark 4.5. For a neo-Hookean solid, in the initial body $\left(R_{1} \leq R \leq R_{0}\right), d \tilde{\sigma}^{r r}(R) / d R$ has the same sign as $\tilde{r}\left(R_{0}\right)-R_{0}$, i.e. the radial component of the Cauchy stress is either strictly increasing or strictly decreasing. Similarly, $d \overline{\tilde{\sigma}}^{\theta \theta}(R) / R$ has the same sign as $-\left(\tilde{r}\left(R_{0}\right)-R_{0}\right)$. In the secondary body $\left(R_{0} \leq R \leq R_{a}\right)$ the critical points of $\tilde{\sigma}^{r r}(R)$ are the zeros of $\tilde{r}(R)-\bar{r}(R)=0$.

\subsubsection{Accretion stresses in the linearized theory}

In this section we calculate the accretion stresses when strains are small. We do this using two approaches. In the first method, we linearize the present nonlinear theory with respect to a trivial stress-free configuration. In the second approach we follow Brown and Goodman [1963]. We will see that the two methods lead to identical solutions as expected.

Linearization of the nonlinear governing equations. In nonlinear elasticity one can linearize the kinematics and the governing equations with respect to any motion. Usually, linearization is done with respect to a stress-free configuration. However, this is not always the case; the so called small-on-large theory of Green et al. [1952] is linearization about a finitely-deformed and stressed configuration. In geometric elasticity, in order to linearize one needs a reference motion $\stackrel{\circ}{\varphi}$ and a one-parameter family of motions $\varphi_{\epsilon}$ such that $\varphi_{\epsilon=0}=\stackrel{\circ}{\varphi}$ [Marsden and Hughes, 1983; Yavari and Ozakin, 2008].

Let us consider a one-parameter family of motions $\varphi_{\epsilon}$ that are assumed to be axisymmetric, i.e., $\varphi_{\epsilon}(R, \Theta)=$ $\left(r_{\epsilon}(R, t), \Theta, t\right)$. Here we linearize about the stress-free configuration $\stackrel{\circ}{\varphi}(R, \Theta)=(R, \Theta)$, i.e., $r_{\epsilon=0}(R, t)=R$. Note that this reference motion corresponds to deformation of a hollow cylindrical bar under no external forces going through accretion by adding stress-free layers to its outer boundary. The variation field is defined as

$$
\delta \varphi_{t}(R, \Theta)=\left.\frac{d}{d \epsilon}\right|_{\epsilon=0} \varphi_{\epsilon}(R, \Theta, t) .
$$

This is what in linear elasticity is called the displacement field. ${ }^{7}$ For the special variations we consider here only the radial displacement component is non-zero and is defined as

$$
\delta r(R, t)=\left.\frac{d}{d \epsilon}\right|_{\epsilon=0} r_{\epsilon}(R, t) .
$$

This implies that $\delta r_{0}(t)=\delta r\left(R_{0}, t\right)$ and $\delta \bar{r}(R)=\delta r\left(R, \frac{R-R_{0}}{u_{0}}\right)$.

\footnotetext{
${ }^{7}$ However, in linear accretion mechanics there is a subtly in defining the displacement field. The displacement field for the new material points is defined with respect to their positions at the time of attachment. For surface growth of a cylindrical bar, this means that $\mathbf{U}(R, \Theta, t)=\delta \varphi_{t}(R, \Theta)-\delta \varphi_{\tau(R)}(R, \Theta)$.
} 
Kinematics. For $R_{1} \leq R \leq R_{0}$, for the perturbed motions we have $r_{\epsilon=0}^{2}(R, t)=r_{\epsilon}^{2}\left(R_{0}, t\right)+R^{2}-R_{2}^{2}$. Taking derivative with respect to $\epsilon$ on both sides and evaluating at $\epsilon=0$ one obtains

$$
\delta r(R, t)=\frac{R_{0}}{R} \delta r_{0}(t),
$$

where $\delta r_{0}(t)=\delta r\left(R_{0}, t\right)$. Knowing that $r(R, 0)=R$, one concludes that $\delta r(R, 0)=0$ (note that for $t=0$, $\left.R_{1} \leq R \leq R_{0}\right)$. For $R_{0} \leq R \leq s(t)$

$$
r_{\epsilon}^{2}(R, t)=r_{\epsilon}^{2}\left(R_{0}, t\right)+\int_{R_{0}}^{R} 2 \bar{r}_{\epsilon}(x) d x
$$

Again taking derivative with respect to $\epsilon$ on both sides and evaluating at $\epsilon=0$ one obtains

$$
\delta r(R, t)=\frac{R_{0}}{R} \delta r_{0}(t)+\frac{1}{R} \int_{R_{0}}^{R} \delta \bar{r}(x) d x .
$$

Evaluating (4.58) at $t=\tau(R)$, one gets

$$
\frac{1}{R} \int_{R_{0}}^{R} \delta \bar{r}(x) d x=\delta \bar{r}(R)-\frac{R_{0}}{R} \delta r_{0}(\tau(R)) .
$$

Substituting the above relation back into (4.58) one has

$$
\delta r(R, t)-\delta \bar{r}(R)=\frac{R_{0}}{R}\left[\delta r_{0}(t)-\delta r_{0}(\tau(R))\right] .
$$

Accretion stresses. Let us start with a neo-Hookean solid and linearize the surface growth-induced stresses. ${ }^{8}$ We consider a one-parameter family of applied internal pressures $\left(p_{i}\right)_{\epsilon}(t)$ and assume that $\left(p_{i}\right)_{\epsilon=0}(t)=0$. With an abuse of notation, we denote the linearized pressure field by $p_{i}(t)$ (instead of $\delta p_{i}(t)$ ). For $R_{1} \leq R \leq R_{0}$, for the perturbed motions from (4.24) we have

$$
\sigma_{\epsilon}^{r r}(R, t)=-\left(p_{i}\right)_{\epsilon}(t)+\int_{R_{1}}^{R} \frac{\mu}{x}\left[1-\frac{x^{4}}{r_{\epsilon}^{4}(x, t)}\right] d x .
$$

Taking derivative with respect to $\epsilon$ on both sides and evaluating at $\epsilon=0$ one obtains

$$
\delta \sigma^{r r}(R, t)=-p_{i}(t)+4 \mu \int_{R_{1}}^{R} \frac{\delta r(R, t)}{x^{2}} d x .
$$

Using (4.56) we have

$$
\delta \sigma^{r r}(R, t)=-p_{i}(t)+2 \mu R_{0}\left(\frac{1}{R_{1}^{2}}-\frac{1}{R^{2}}\right) \delta r_{0}(t) .
$$

For $R_{0} \leq R \leq R_{0}+u_{0} t$, from (4.28) one has

$$
\sigma_{\epsilon}^{r r}(R, t)=\sigma_{\epsilon}^{r r}\left(R_{0}, t\right)+\int_{R_{0}}^{R} \frac{\mu}{\bar{r}_{\epsilon}(x)}\left[1-\frac{\bar{r}_{\epsilon}^{4}(x)}{r_{\epsilon}^{4}(x, t)}\right] d x .
$$

Therefore

$$
\delta \sigma^{r r}(R, t)=-p_{i}(t)+2 \mu R_{0}\left(\frac{1}{R_{1}^{2}}-\frac{1}{R_{0}^{2}}\right) \delta r_{0}(t)+4 \mu \int_{R_{0}}^{R} \frac{\delta r(x, t)-\delta \bar{r}(x)}{x^{2}} d x
$$

\footnotetext{
${ }^{8}$ In this particular problem, for an arbitrary isotropic incompressible solid instead of $\mu$ one would have the following constant

$$
\left.2\left(\frac{\partial W}{\partial I_{1}}+\frac{\partial W}{\partial I_{2}}\right)\right|_{I_{1}=2, I_{2}=1} .
$$
}


and using (4.60) we obtain

$$
\delta \sigma^{r r}(R, t)=-p_{i}(t)+2 \mu R_{0}\left(\frac{1}{R_{1}^{2}}-\frac{1}{R^{2}}\right) \delta r_{0}(t)-4 \mu R_{0} \int_{R_{0}}^{R} \frac{\delta r_{0}(\tau(x))}{x^{3}} d x .
$$

We know that $\sigma_{\epsilon}^{r r}(s(t), t)=0$ and hence $\delta \sigma^{r r}(s(t), t)=0$. Therefore

$$
\left(\frac{1}{R_{1}^{2}}-\frac{1}{s^{2}(t)}\right) \delta r_{0}(t)-2 \int_{R_{0}}^{s(t)} \frac{\delta r_{0}(\tau(x))}{x^{3}} d x=\frac{p_{i}(t)}{2 \mu R_{0}} .
$$

Taking derivative with respect to $t$ of both sides one reduces the above integral equation to the following simple ODE:

$$
\frac{\dot{\delta r_{0}}}{\delta(t)}=\frac{R_{1}^{2}}{2 \mu R_{0}} \frac{s^{2}(t) \dot{p}_{i}(t)}{s^{2}(t)-R_{1}^{2}} .
$$

Integrating this ODE from 0 to $t$ and noting that $\dot{\delta r}\left(R_{0}, 0\right)=0$, one obtains

$$
\delta r_{0}(t)=\frac{R_{1}^{2}}{2 \mu R_{0}} \int_{0}^{t} \frac{s^{2}(\eta) \dot{p}_{i}(\eta)}{s^{2}(\eta)-R_{1}^{2}} d \eta
$$

Therefore, substituting into (4.60), we have

$$
\delta r(R, t)-\delta \bar{r}(R)=\frac{R_{1}^{2}}{2 \mu R} \int_{\tau(R)}^{t} \frac{s^{2}(\eta) \dot{p}_{i}(\eta)}{s^{2}(\eta)-R_{1}^{2}} d \eta .
$$

As was mentioned earlier, the left-hand side of the above relation is what is defined to be the radial displacement in linear surface growth mechanics. Note that once the difference $\delta r(R, t)-\delta \bar{r}(R)$ is known, one can calculate $\delta r(R, t)$ by solving Eq. (4.59) for $\delta \bar{r}(R)$.

Taking time derivative of both sides of (4.66) we obtain

$$
\frac{\dot{\delta \sigma^{r r}}}{\delta}(R, t)=-\dot{p}_{i}(t)+2 \mu R_{0}\left(\frac{1}{R_{1}^{2}}-\frac{1}{R^{2}}\right) \dot{\delta r_{0}}(t)
$$

Integrating the above relation from $\tau(R)$ to $t$ and using the condition $\delta \sigma^{r r}(R, \tau(R))=0$, we have

$$
\delta \sigma^{r r}(R, t)=-p_{i}(t)+p_{i}(\tau(R))+\left(1-\frac{R_{1}^{2}}{R^{2}}\right) \int_{\tau(R)}^{t} \frac{s^{2}(\eta) \dot{p}_{i}(\eta)}{s^{2}(\eta)-R_{1}^{2}} d \eta
$$

Linearizing (4.30) we get $\left(\bar{\sigma}^{\theta \theta}=r^{2} \sigma^{\theta \theta}\right.$ is the physical circumferential component)

$$
\delta \bar{\sigma}^{\theta \theta}(R, t)=\delta \bar{\sigma}^{r r}(R, t)+\frac{4 \mu}{R}[\delta r(R, t)-\delta \bar{r}(R)]=\delta \bar{\sigma}^{r r}(R, t)+\frac{2 R_{1}^{2}}{R^{2}} \int_{\tau(R)}^{t} \frac{s^{2}(\eta) \dot{p}_{i}(\eta)}{s^{2}(\eta)-R_{1}^{2}} d \eta,
$$

which leads to

$$
\delta \bar{\sigma}^{\theta \theta}(R, t)=-p_{i}(t)+p_{i}(\tau(R))+\left(1+\frac{R_{1}^{2}}{R^{2}}\right) \int_{\tau(R)}^{t} \frac{s^{2}(\eta) \dot{p}_{i}(\eta)}{s^{2}(\eta)-R_{1}^{2}} d \eta
$$

Calculation of accretion stresses using the linear theory of Brown and Goodman [1963]. Next we solve the symmetric accretion problem of a hollow cylindrical bar in the setting of linear elasticity. We follow Brown and Goodman [1963], but instead of using the external radius $s(t)=R_{0}+u_{0} t$ as the independent variable we use time. We indicate with $U^{R}(R, t)$ the radial displacement, with $p_{i}(t)$ the internal pressure, with $\boldsymbol{\sigma}(R, t)$ the Cauchy stress tensor, and with $p(R, t)$ the pressure field. All these quantities are considered in the framework of linearized elasticity. 
In the initial body, i.e, for $R_{1} \leq R \leq R_{0}$, where strains are compatible, the linear problem consists of the following governing equations:

$$
\begin{cases}\frac{\partial U^{R}}{\partial R}(R, t)+\frac{U^{R}(R, t)}{R}=0 & \text { (incompressibility condition), } \\ \sigma^{R R}(R, t)=2 \mu \frac{\partial U^{R}}{\partial R}(R, t)-p(R, t), \sigma^{\Theta \Theta}(R, t)=2 \mu \frac{U^{R}(R, t)}{R^{3}}-\frac{p(R, t)}{R^{2}} & \text { (constitutive relations), } \\ \frac{\partial \sigma^{R R}}{\partial R}(R, t)+\frac{\sigma^{R R}(R, t)}{R}-R \sigma^{\Theta \Theta}(R, t)=0 & \text { (radial equilibrium equation). }\end{cases}
$$

The boundary and continuity conditions are $\sigma^{R R}\left(R_{1}, t\right)=-p_{i}(t)$ and $\sigma^{R R}\left(R_{0}^{-}, t\right)=\sigma^{R R}\left(R_{0}^{+}, t\right)$. From the incompressibility condition we can write the radial displacement as

$$
U^{R}(R, t)=U_{0}^{R}(t) \frac{R_{0}}{R}
$$

where $U_{0}^{R}(t)=U^{R}\left(R_{0}, t\right)$ is an unknown function to be determined. Therefore, the equilibrium equation is simplified to read

$$
\frac{\partial \sigma^{R R}(R, t)}{\partial R}=4 \mu \frac{R_{0} U_{0}^{R}(t)}{R^{3}}
$$

which, taking into account the traction boundary condition at $R=R_{1}$, leads to

$$
\sigma^{R R}(R, t)=-p_{i}(t)+\int_{R_{1}}^{R} 4 \mu \frac{R_{0} U_{0}^{R}(t)}{x^{3}} d x=-p_{i}(t)+2 \mu R_{0}\left(\frac{1}{R_{1}^{2}}-\frac{1}{R^{2}}\right) U_{0}^{R}(t) .
$$

From $(4.75)_{2}$ we know that $\bar{\sigma}^{\Theta \Theta}(R, t)=\sigma^{R R}+4 \mu U^{R} / R$, which gives us

$$
\bar{\sigma}^{\Theta \Theta}(R, t)=-p_{i}(t)+2 \mu R_{0}\left(\frac{1}{R_{1}^{2}}+\frac{1}{R^{2}}\right) U^{R}\left(R_{0}, t\right) .
$$

Now one can write $\sigma^{R R}\left(R_{0}, t\right)$, which is denoted by $\sigma_{0}(t)$, as

$$
\sigma_{0}(t)=\sigma^{R R}\left(R_{0}, t\right)=-p_{i}(t)+2 \mu R_{0}\left(\frac{1}{R_{1}^{2}}+\frac{1}{R_{0}^{2}}\right) U_{0}^{R}(t) .
$$

For $R_{0} \leq R \leq R_{0}+u_{0} t=s(t)$, following [Brown and Goodman, 1963], the incremental problem for the growing incompressible cylinder at time $t$ is written as:

$$
\begin{cases}\frac{\partial \dot{U}^{R}}{\partial R}(R, t)+\frac{\dot{U}^{R}(R, t)}{R}=0 & \text { (incompressibility condition), } \\ \dot{\sigma}^{R R}(R, t)=2 \mu \frac{\partial \dot{U}^{R}}{\partial R}(R, t)-\dot{p}(R, t), \quad \dot{\sigma}^{\Theta \Theta}(R, t)=2 \mu \frac{\dot{U}^{R}(R, t)}{R^{3}}-\frac{\dot{p}(R, t)}{R^{2}} & \text { (consitituive relations), } \\ \frac{\partial \dot{\sigma}^{R R}}{\partial R}(R, t)+\frac{\dot{\sigma}^{R R}(R, t)}{R}-R \dot{\sigma}^{\Theta \Theta}(R, t)=0 & \text { (radial equilibrium equation). }\end{cases}
$$

The boundary conditions for this incremental problem are $\dot{\sigma}^{R R}\left(R_{0}, t\right)=\dot{\sigma}_{0}(t)$ and $\dot{\sigma}^{R R}(s(t), t)=0$, where $\dot{\sigma}_{0}(t)$ is the rate of the radial stress in (4.80) at the interface with the initial body, i.e.

$$
\dot{\sigma}_{0}(t)=-\dot{p}_{i}(t)+2 \mu R_{0}\left(\frac{1}{R_{1}^{2}}-\frac{1}{R_{0}^{2}}\right) \dot{U}_{0}^{R}(t) .
$$

Therefore, from Eqs. (4.76), (4.78) and (4.79) one obtains

$$
\begin{aligned}
\dot{U}^{R}(R, t) & =\frac{R_{0}}{R} \dot{U}_{0}^{R}(t), \\
\dot{\sigma}^{R R}(R, t) & =\dot{\sigma}_{0}(t)+2 \mu R_{0}\left(\frac{1}{R_{0}^{2}}-\frac{1}{R^{2}}\right) \dot{U}_{0}^{R}(t)=-\dot{p}_{i}(t)+2 \mu R_{0}\left(\frac{1}{R_{1}^{2}}-\frac{1}{R^{2}}\right) \dot{U}_{0}^{R}(t), \\
\dot{\bar{\sigma}}^{\Theta \Theta}(R, t) & =\dot{\sigma}_{0}(t)+2 \mu R_{0}\left(\frac{1}{R_{0}^{2}}+\frac{1}{R^{2}}\right) \dot{U}_{0}^{R}(t)=-\dot{p}_{i}(t)+2 \mu R_{0}\left(\frac{1}{R_{1}^{2}}+\frac{1}{R^{2}}\right) \dot{U}_{0}^{R}(t) .
\end{aligned}
$$


The function $U_{0}^{R}(t)$ can now be obtained using the condition $\dot{\sigma}^{R R}(s(t), t)=0$, i.e.

$$
\dot{U}_{0}^{R}(t)=\frac{R_{1}^{2}}{2 \mu R_{0}} \frac{s^{2}(t) \dot{p}_{i}(t)}{s^{2}(t)-R_{1}^{2}} .
$$

Note that

$$
\dot{U}_{0}^{R}(t)=\dot{\overline{\delta r}}\left(R_{0}, t\right), \quad \dot{\sigma}^{R R}(R, t)=\dot{\dot{\delta \sigma^{r r}}}(R, t), \quad \dot{\bar{\sigma}}^{\Theta \Theta}(R, t)=\dot{\dot{\delta \bar{\sigma}^{\theta \theta}}}(R, t) .
$$

Moreover, the total displacement and stress fields are given by

$$
U^{R}(R, t)=U^{R}(R, \tau(R))+\int_{\tau(R)}^{t} \dot{U}^{R}(R, \eta) d \eta, \quad \boldsymbol{\sigma}(R, t)=\boldsymbol{\sigma}(R, \tau(R))+\int_{\tau(R)}^{t} \dot{\boldsymbol{\sigma}}(R, \eta) d \eta,
$$

where $\sigma^{R R}(R, \tau(R))=0, \sigma^{\Theta \Theta}(R, \tau(R))=0$, and $U^{R}(R, \tau(R))=0$. Therefore, as expected, the two approaches lead to identical solutions.

Residual stresses. Integrating Eqs. (4.70), (4.72) and (4.74) by parts, one obtains

$$
\begin{aligned}
U^{R}(R, t) & =\frac{R_{1}^{2}}{2 \mu R} \frac{s^{2}(t) p_{i}(t)}{s^{2}(t)-R_{1}^{2}}-\frac{R_{1}^{2} R}{R^{2}-R_{1}^{2}} \frac{p_{i}(\tau(R))}{2 \mu}+\frac{u_{0} R_{1}^{4}}{\mu R} \int_{\tau(R)}^{t} \frac{s(\eta) p_{i}(\eta)}{\left(s^{2}(\eta)-R_{1}^{2}\right)^{2}} d \eta, \\
\sigma^{R R}(R, t) & =-\frac{R_{1}^{2}}{R^{2}} \frac{s^{2}(t)-R^{2}}{s^{2}(t)-R_{1}^{2}} p_{i}(t)+2 u_{0} R_{1}^{2}\left(1-\frac{R_{1}^{2}}{R^{2}}\right) \int_{\tau(R)}^{t} \frac{s(\eta) p_{i}(\eta)}{\left(s^{2}(\eta)-R_{1}^{2}\right)^{2}} d \eta, \\
\bar{\sigma}^{\Theta \Theta}(R, t) & =\frac{R_{1}^{2}}{R^{2}} \frac{s^{2}(t)+R^{2}}{s^{2}(t)-R_{1}^{2}} p_{i}(t)-\frac{2 R_{1}^{2}}{R^{2}-R_{1}^{2}} p_{i}(\tau(R))+2 u_{0} R_{1}^{2}\left(1+\frac{R_{1}^{2}}{R^{2}}\right) \int_{\tau(R)}^{t} \frac{s(\eta) p_{i}(\eta)}{\left(s^{2}(\eta)-R_{1}^{2}\right)^{2}} d \eta .
\end{aligned}
$$

If at time $t_{a}$ we stop surface growth and remove the internal applied pressure, we would observe residual stresses in both the initial and the secondary bodies. In the linear theory of accretion these stresses can be calculated using superposition. For the cylinder of internal radius $R_{1}$ and external radius $s\left(t_{a}\right)=R_{0}+u_{0} t_{a}$ loaded by an internal pressure $-p_{i}\left(t_{a}\right)$ (unloading) and traction-free at the external radius one has

$$
\begin{aligned}
U_{\text {unloading }}^{R}\left(R, t_{a}\right) & =-\frac{1}{2} \frac{R_{1}^{2}}{R} \frac{s^{2}\left(t_{a}\right)}{s^{2}\left(t_{a}\right)-R_{1}^{2}} \frac{p_{i}\left(t_{a}\right)}{\mu}, \\
\sigma_{\text {unloading }}^{R R}\left(R, t_{a}\right) & =\frac{R_{1}^{2}}{R^{2}} \frac{s^{2}\left(t_{a}\right)-R^{2}}{s^{2}\left(t_{a}\right)-R_{1}^{2}} p_{i}\left(t_{a}\right), \\
\bar{\sigma}_{\text {unloading }}^{\Theta \Theta}\left(R, t_{a}\right) & =-\frac{R_{1}^{2}}{R^{2}} \frac{s^{2}\left(t_{a}\right)+R^{2}}{s^{2}\left(t_{a}\right)-R_{1}^{2}} p_{i}\left(t_{a}\right) .
\end{aligned}
$$

Adding the above to their corresponding values at the end of loading one obtains the residual displacement and the residual stresses as:

$$
\begin{aligned}
& U_{\mathrm{res}}^{R}\left(R, t_{a}\right)=-\frac{R_{1}^{2} R}{R^{2}-R_{1}^{2}} \frac{p_{i}(\tau(R))}{2 \mu}+\frac{u_{0} R_{1}^{4}}{\mu R} \int_{\frac{R-R_{0}}{u_{0}}}^{t_{a}} \frac{s(\eta) p_{i}(\eta)}{\left(s^{2}(\eta)-R_{1}^{2}\right)^{2}} d \eta \\
& \sigma_{\mathrm{res}}^{R R}\left(R, t_{a}\right)=2 u_{0} R_{1}^{2}\left(1-\frac{R_{1}^{2}}{R^{2}}\right) \int_{\frac{R-R_{0}}{u_{0}}}^{t_{a}} \frac{s(\eta) p_{i}(\eta)}{\left(s^{2}(\eta)-R_{1}^{2}\right)^{2}} d \eta \\
& \bar{\sigma}_{\mathrm{res}}^{\Theta \Theta}\left(R, t_{a}\right)=-2 \frac{R_{1}^{2}}{R^{2}-R_{1}^{2}} p_{i}\left(\frac{R-R_{0}}{u_{0}}\right)+2 u_{0} R_{1}^{2}\left(1+\frac{R_{0}^{2}}{R^{2}}\right) \int_{\frac{R-R_{0}}{u_{0}}}^{t_{a}} \frac{s(\eta) p_{i}(\eta)}{\left(s^{2}(\eta)-R_{1}^{2}\right)^{2}} d \eta .
\end{aligned}
$$

\subsection{Inner Accretion}

In this section we consider an infinitely-long thick hollow cylinder under a specified time-dependent internal pressure $p_{i}(t)$. Initially the inner and outer radii of the cylinder are $R_{1}$ and $R_{0}$, respectively. We assume that material is continuously added to the cylinder on its inner boundary. The outer boundary is assumed to be traction free (see Fig.4). 


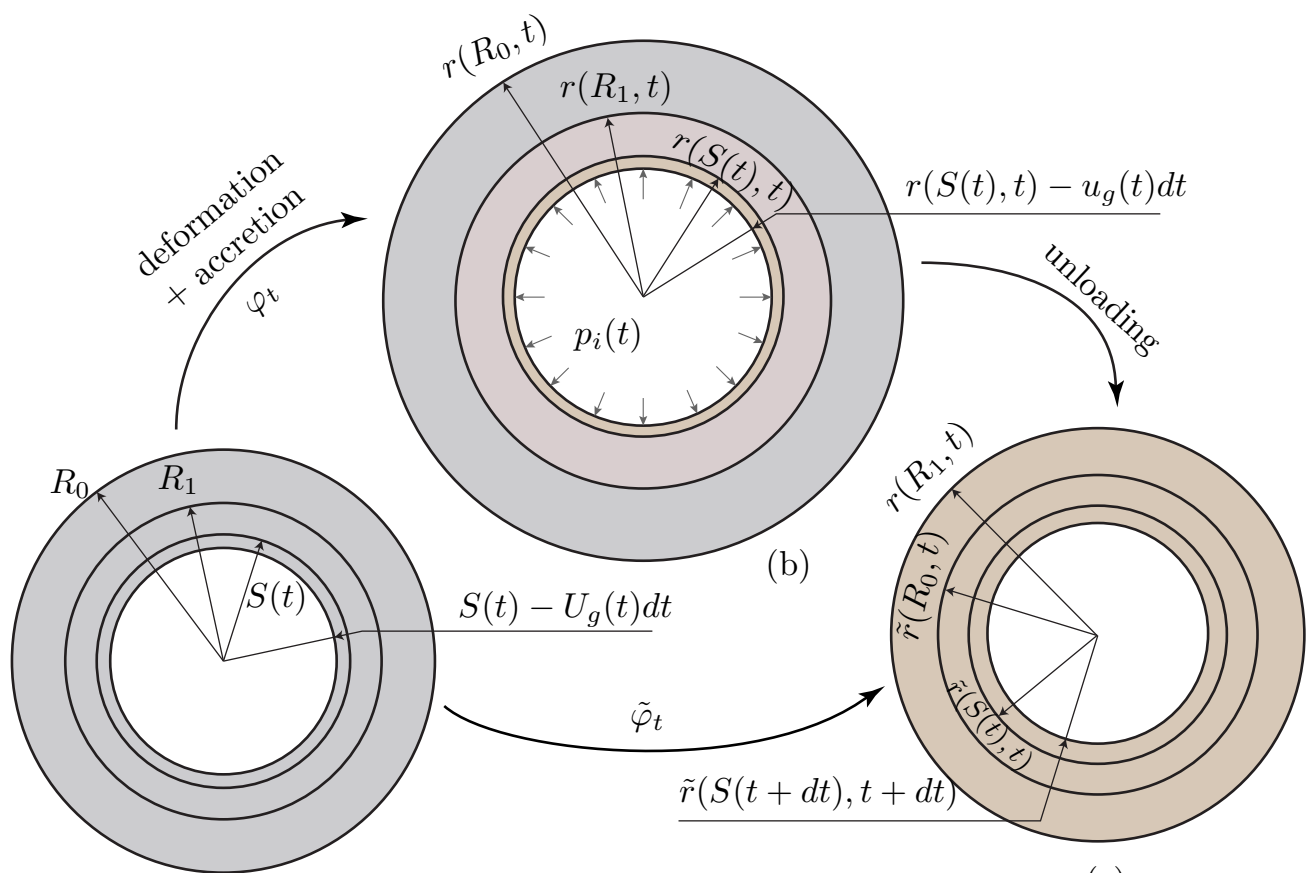

(a)

(c)

Figure 4: Continuous Inner Accretion: (a) The material manifold $(\mathcal{B}, \mathbf{G})$. The inner radius at time $t$ is $S(t)$. At a later time $t+d t$ the inner radius is changed to $S(t)-U_{g}(t) d t$. (b) The deformed annulus under a specified internal pressure $p_{i}(t)$ with a layer of stress-feee material of thickness $u_{g}(t) d t$ attached to it during the time interval $[t, t+d t]$. (c) The residually-stressed accreted body after removal of external forces (here the internal pressure $p_{i}(t)$ ).

\subsubsection{Kinematics and material metric}

Again, for the sake of simplicity of calculations, we assume a constant growth velocity $u_{1}>0$ and work with the choice $U_{g}(t)=u_{g}(t)=u_{1}$. In this problem the time-dependent material manifold is

$$
\mathcal{B}_{t}=\left\{(R, \Theta): 0 \leq \Theta<2 \pi, S(t)=R_{1}-u_{1} t \leq R \leq R_{0}\right\} .
$$

Note that in order to avoid interpenetration of matter we assume that $0 \leq t<\frac{R_{1}}{u_{1}}$.

The ambient space metric and the deformation gradient have the same forms as those of the outer accretion example. Again, we assign to each layer at radius $R$ in the material manifold a time of accretion $\tau(R)$, which is assumed to be invertible with inverse $S=\tau^{-1}$. We assume that $\tau\left(R_{1}\right)=0$. Note that $u_{g}(t)=r^{\prime}(S(t), t) U_{g}(t)$, where $U_{g}(t)=\dot{S}(t)$. Equivalently, $r^{\prime}(R, \tau(R))=\tau^{\prime}(R) u_{g}(\tau(R))$. The choice $U_{g}(t)=u_{g}(t)$ implies that $r^{\prime}(S(t), t)=1$ or equivalently $r^{\prime}(R, \tau(R))=1$. For constant growth velocity $u_{g}(t)=u_{1}>0$ one has

$$
S(t)=R_{1}-u_{1} t, \text { or } \quad \tau(R)=\frac{R_{1}-R}{u_{1}} .
$$

For $R_{1} \leq R \leq R_{0}$, the material metric is flat and has the representation $\mathbf{G}=\operatorname{diag}\left\{1, R^{2}\right\}$ in polar coordinates.

The material points attached to the growth surface at time $t$ are in a state of hydrostatic pressure with magnitude $p_{i}(t)$ (when $p_{i}(t)<0$ this would be a hydrostatic suction). In the time interval $[t, t+d t]$ a ring of radius $r(S(t), t)$ with thickness $u_{1} d t$ is attached to the deformed body. Assuming that the body and the attached particles are made of an incompressible material, if this ring (which is in a state of hydrostatic pressure or suction) is unloaded its radius would remain $r(S(t), t)$, i.e. $r(S(t), t)$ is the radius of the accreted ring in its stress-free configuration as well. Therefore, for $S(t) \leq R \leq R_{1}$, we have

$$
\mathbf{G}(S(t))=\varphi_{t}^{*} \mathbf{g}(r(S(t), t)) \text {, or equivalently } \quad \mathbf{G}(R)=\varphi_{\tau(R)}^{*} \mathbf{g}(r(R, \tau(R))) .
$$

Hence

$$
\mathbf{G}=\left(\begin{array}{cc}
1 & 0 \\
0 & r^{2}(R, \tau(R))
\end{array}\right)
$$


For $u_{g}(t)=u_{1}$, this is simplified to read

$$
\mathbf{G}(R)=\left(\begin{array}{cc}
1 & 0 \\
0 & r^{2}\left(R, \frac{R_{1}-R}{u_{1}}\right)
\end{array}\right) .
$$

Therefore, in summary, the material manifold is an evolving Riemannian manifold $\left(\mathcal{B}_{t}, \mathbf{G}\right)$, where

$$
\mathcal{B}_{t}=\left\{(R, \Theta): 0 \leq \Theta<2 \pi, S(t) \leq R \leq R_{0}\right\}
$$

and

$$
\begin{aligned}
S(t) \leq R \leq R_{1}: \quad \mathbf{G}=\left(\begin{array}{cc}
1 & 0 \\
0 & r^{2}\left(R, \frac{R_{1}-R}{u_{1}}\right)
\end{array}\right), \\
R_{1} \leq R \leq R_{0}: \quad \mathbf{G}=\left(\begin{array}{cc}
1 & 0 \\
0 & R^{2}
\end{array}\right) .
\end{aligned}
$$

Let us define $\hat{r}(R):=r\left(R, \frac{R_{1}-R}{u_{1}}\right)$. Using the incompressibility constraint we obtain

$$
\begin{gathered}
S(t) \leq R \leq R_{1}: \quad r^{2}(R, t)=r^{2}\left(R_{1}, t\right)-\int_{R}^{R_{1}} 2 \hat{r}(x) d x, \\
R_{1} \leq R \leq R_{0}: \quad r^{2}(R, t)=r^{2}\left(R_{1}, t\right)-R_{1}^{2}+R^{2} .
\end{gathered}
$$

\subsubsection{Calculation of stresses for an incompressible isotropic accreting cylinder}

The stress calculation is very similar to that of the outer accretion problem. For $R_{1} \leq R \leq R_{0}$, the radial Cauchy stress component has the following expression (the boundary condition $\sigma^{r r}\left(R_{0}, t\right)=0$ has been enforced):

$$
\sigma^{r r}(R, t)=-\int_{R}^{R_{0}} \frac{\alpha(x, t)+\beta(x, t)}{x}\left[1-\frac{x^{4}}{r^{4}(x, t)}\right] d x .
$$

For $S(t) \leq R \leq R_{1}$, the radial Cauchy stress component reads

$$
\begin{aligned}
\sigma^{r r}(R, t) & =\sigma^{r r}\left(R_{1}, t\right)-\int_{R}^{R_{1}} \frac{\alpha(x, t)+\beta(x, t)}{\hat{r}(x)}\left[1-\frac{\hat{r}^{4}(x)}{r^{4}(x, t)}\right] d x \\
& =-\int_{R_{1}}^{R_{0}} \frac{\alpha(x, t)+\beta(x, t)}{x}\left[1-\frac{x^{4}}{r^{4}(x, t)}\right] d x-\int_{R}^{R_{1}} \frac{\alpha(x, t)+\beta(x, t)}{\hat{r}(x)}\left[1-\frac{\hat{r}^{4}(x)}{r^{4}(x, t)}\right] d x .
\end{aligned}
$$

Note that $\sigma^{r r}(S(t), t)=-p_{i}(t)$ and thus

$$
\int_{R_{1}}^{R_{0}} \frac{\alpha(x, t)+\beta(x, t)}{x}\left[1-\frac{x^{4}}{r^{4}(x, t)}\right] d x+\int_{S(t)}^{R_{1}} \frac{\alpha(x, t)+\beta(x, t)}{\hat{r}(x)}\left[1-\frac{\hat{r}^{4}(x)}{r^{4}(x, t)}\right] d x=p_{i}(t) .
$$

Note that similar to the outer accretion problem, $\sigma^{r r}(S(t), t)=-p_{i}(t)$ implies that $\bar{\sigma}^{\theta \theta}(S(t), t)=-p_{i}(t)$.

In the case of a homogeneous neo-Hookean solid, (4.106) is simplified to read

$$
\int_{R_{1}}^{R_{0}} \frac{1}{x}\left[1-\frac{x^{4}}{r^{4}(x, t)}\right] d x+\int_{S(t)}^{R_{1}} \frac{1}{\hat{r}(x)}\left[1-\frac{\hat{r}^{4}(x)}{r^{4}(x, t)}\right] d x=\frac{p_{i}(t)}{\mu} .
$$

Eq. (4.107) can be expressed in terms of the function $r(R, t)$ and its spatial derivative $r^{\prime}(R, t)$, leading to the following problem in the triangular region $\left\{(R, t) \in \mathbb{R}^{2} \mid R_{1}-u_{1} t \leq R \leq R_{1}, 0<t<\frac{R_{1}}{u_{1}}\right\}$ :

$$
\left\{\begin{array}{l}
r(R, t) r^{\prime}(R, t)=r\left(R, \frac{R_{1}-R}{u_{1}}\right), \\
\int_{R_{1}}^{R_{0}} \frac{1}{x}\left[1-\frac{x^{4}}{\left(r^{2}\left(R_{1}, t\right)+x^{2}-R_{1}^{2}\right)^{2}}\right] d x+\int_{R_{1}-u_{1} t}^{R_{1}} \frac{1-r^{\prime 4}(x, t)}{r(x, t) r^{\prime}(x, t)} d x=\frac{p_{i}(t)}{\mu}, \\
r\left(R_{1}, 0\right)=R_{1} .
\end{array}\right.
$$

Note that the condition $r\left(R_{1}, 0\right)=R_{1}$ implies that for $R_{1} \leq R \leq R_{0}, r(R, 0)=R$. We also assume that $p_{i}(0)=0$. 


\subsubsection{Calculation of residual stresses for an incompressible isotropic accreting cylinder}

After the completion of inner accretion at time $t=t_{a}<R_{1} / u_{1}$, the material metric has the following representation in polar coordinates:

$$
\begin{aligned}
& R_{a} \leq R \leq R_{1}: \quad \mathbf{G}=\left(\begin{array}{cc}
1 & 0 \\
0 & r^{2}\left(R, \frac{R_{1}-R}{u_{1}}\right)
\end{array}\right), \\
& R_{1} \leq R \leq R_{0}: \quad \mathbf{G}=\left(\begin{array}{cc}
1 & 0 \\
0 & R^{2}
\end{array}\right),
\end{aligned}
$$

where $R_{a}=R_{1}-u_{1} t_{a}$. Again, the deformation from the material manifold to the residually-stressed configuration (under no applied loads) is denoted by $\tilde{\varphi}: \mathcal{B} \rightarrow \mathcal{S}$, where in polar coordinates we have: $\tilde{\varphi}(R, \Theta)=(\tilde{r}, \tilde{\theta})=$ $(\tilde{r}(R), \Theta)$, see Fig.4. Using the incompressibility constraint one obtains

$$
\begin{array}{ll}
R_{a} \leq R \leq R_{1}: \quad & \tilde{r}^{2}(R)=\tilde{r}^{2}\left(R_{1}\right)-\int_{R}^{R_{1}} 2 \hat{r}(x) d x, \\
R_{1} \leq R \leq R_{0}: & \tilde{r}^{2}(R)=\tilde{r}^{2}\left(R_{1}\right)-R_{1}^{2}+R^{2},
\end{array}
$$

where $\tilde{r}\left(R_{1}\right)$ is an unknown that will be determined after enforcing the boundary and continuity conditions. For the residual deformation mapping $\tilde{\varphi}$ we have

$$
\begin{array}{lll}
I_{1}=\frac{\tilde{r}^{2}(R)}{\hat{r}^{2}(R)}+\frac{\hat{r}^{2}(R)}{\tilde{r}^{2}(R)}, \quad I_{2}=1, & R_{a} \leq R \leq R_{1}, \\
I_{1}=\frac{\tilde{r}^{2}(R)}{R^{2}}+\frac{R^{2}}{\tilde{r}^{2}(R)}, & I_{2}=1, & R_{1} \leq R \leq R_{0} .
\end{array}
$$

In the absence of applied loads the boundary conditions read: $\tilde{\sigma}^{r r}\left(R_{a}\right)=\tilde{\sigma}^{r r}\left(R_{0}\right)=0$. One then has the following distribution of radial Cauchy stress (the continuity of traction $\tilde{\sigma}^{r r}\left(R_{1}^{-}\right)=\tilde{\sigma}^{r r}\left(R_{1}^{+}\right)$has been enforced):

$$
\begin{aligned}
& R_{a} \leq R \leq R_{1}: \quad \tilde{\sigma}^{r r}(R)=-\int_{R_{1}}^{R_{0}} \frac{\tilde{\alpha}(x)+\tilde{\beta}(x)}{x}\left[1-\frac{x^{4}}{\tilde{r}^{4}(x)}\right] d x-\int_{R}^{R_{1}} \frac{\tilde{\alpha}(x)+\tilde{\beta}(x)}{\hat{r}(x)}\left[1-\frac{\hat{r}^{4}(x)}{\tilde{r}^{4}(x)}\right] d x, \\
& R_{1} \leq R \leq R_{0}: \quad \tilde{\sigma}^{r r}(R)=-\int_{R}^{R_{0}} \frac{\tilde{\alpha}(x)+\tilde{\beta}(x)}{x}\left[1-\frac{x^{4}}{\tilde{r}^{4}(x)}\right] d x .
\end{aligned}
$$

Similarly, for the physical circumferential Cauchy stress component we have

$$
\begin{aligned}
& R_{a} \leq R \leq R_{1}: \quad \overline{\tilde{\sigma}}^{\theta \theta}(R)=\tilde{\sigma}^{r r}(R)+[\tilde{\alpha}(R)+\tilde{\beta}(R)]\left[\frac{\tilde{r}^{2}(R)}{\hat{r}^{2}(R)}-\frac{\hat{r}^{2}(R)}{\tilde{r}^{2}(R)}\right], \\
& R_{1} \leq R \leq R_{0}: \quad \overline{\tilde{\sigma}}^{\theta \theta}(R)=\tilde{\sigma}^{r r}(R)+[\tilde{\alpha}(R)+\tilde{\beta}(R)]\left[\frac{\tilde{r}^{2}(R)}{R^{2}}-\frac{R^{2}}{\tilde{r}^{2}(R)}\right] .
\end{aligned}
$$

The unknown constant $\tilde{r}\left(R_{0}\right)$ is determined using the condition $\tilde{\sigma}^{r r}\left(R_{a}\right)=0$, which is written as

$$
\int_{R_{1}}^{R_{0}} \frac{\tilde{\alpha}(x)+\tilde{\beta}(x)}{x}\left[1-\frac{x^{4}}{\tilde{r}^{4}(x)}\right] d x+\int_{R_{a}}^{R_{1}} \frac{\tilde{\alpha}(x)+\tilde{\beta}(x)}{\hat{r}(x)}\left[1-\frac{\hat{r}^{4}(x)}{\tilde{r}^{4}(x)}\right] d x=0 .
$$

\subsubsection{Accretion stresses in the linearized theory}

Next we calculate the accretion stresses when strains are small. We consider a one-parameter family of motions $\varphi_{\epsilon}$ that are assumed to be axisymmetric, i.e., $\varphi_{\epsilon}(R, \Theta)=\left(r_{\epsilon}(R, t), \Theta, t\right)$ and linearize about the stress-free configuration $\stackrel{\circ}{\varphi}(R, \Theta)=(R, \Theta)$, i.e., $r_{\epsilon=0}(R, t)=R$. For $R_{1} \leq R \leq R_{0}$, the perturbed motion reads $r_{\epsilon}^{2}(R, t)=$ $r_{\epsilon}^{2}\left(R_{1}, t\right)-R_{1}^{2}+R^{2}$. Taking derivative with respect to $\epsilon$, and evaluating at $\epsilon=0$, we obtain

$$
\delta r(R, t)=\frac{R_{1}}{R} \delta r_{1}(t)
$$


where $\delta r_{1}(t)=\delta r\left(R_{1}, t\right)$. Knowing that $r(R, 0)=R$, one concludes that $\delta r(R, 0)=0$. Note that for $t=0$, $R_{1} \leq R \leq R_{0}$. For $S(t) \leq R \leq R_{1}$ we have

$$
r_{\epsilon}^{2}(R, t)=r_{\epsilon}^{2}\left(R_{1}, t\right)-\int_{R}^{R_{1}} 2 \hat{r}_{\epsilon}(x) d x
$$

Thus

$$
\delta r(R, t)=\frac{R_{1}}{R} \delta r_{1}(t)-\frac{1}{R} \int_{R}^{R_{1}} \delta \hat{r}(x) d x .
$$

Evaluating $(4.117)_{1}$ at $t=\tau(R)$, one obtains

$$
-\frac{1}{R} \int_{R}^{R_{1}} \delta \hat{r}(x) d x=\delta \hat{r}(R)-\frac{R_{1}}{R} \delta r_{1}(\tau(R)) .
$$

Substituting the above relation back into (4.117) one gets

$$
\delta r(R, t)-\delta \hat{r}(R)=\frac{R_{1}}{R}\left[\delta r_{1}(t)-\delta r_{1}(\tau(R))\right] .
$$

Let us define

$$
\mu=\left.2\left(\frac{\partial W}{\partial I_{1}}+\frac{\partial W}{\partial I_{2}}\right)\right|_{I_{1}=2, I_{2}=1} .
$$

Similar to the outer accretion problem, we consider a one-parameter family of applied internal pressures $\left(p_{i}\right)_{\epsilon}(t)$ $\left(\left(p_{i}\right)_{\epsilon=0}(t)=0\right)$ and with an abuse of notation denote the linearized pressure field by $p_{i}(t)$. Similar to the linearization of stresses for the outer accretion problem we have the following linearized radial Cauchy stress. For $R_{1} \leq R \leq R_{0}$, using (4.115), we obtain

$$
\delta \sigma^{r r}(R, t)=-4 \mu \int_{R}^{R_{0}} \frac{\delta r(x, t)}{x^{2}} d x=-2 \mu R_{1}\left(\frac{1}{R^{2}}-\frac{1}{R_{0}^{2}}\right) \delta r_{1}(t) .
$$

For $S(t) \leq R \leq R_{1}$,

$$
\delta \sigma^{r r}(R, t)=-4 \mu \int_{R_{1}}^{R_{0}} \frac{\delta r(x, t)}{x^{2}} d x-4 \mu \int_{R}^{R_{1}} \frac{\delta r(x, t)-\delta \hat{r}(x)}{x^{2}} d x .
$$

Using (4.117), we can simplify the above equation as

$$
\delta \sigma^{r r}(R, t)=-2 \mu R_{1}\left(\frac{1}{R_{1}^{2}}-\frac{1}{R_{0}^{2}}\right) \delta r_{1}(t)-4 \mu \int_{R}^{R_{1}} \frac{\delta r(x, t)-\delta \hat{r}(x)}{x^{2}} d x .
$$

From (4.119) one obtains

$$
\delta \sigma^{r r}(R, t)=-2 \mu R_{1}\left(\frac{1}{R}-\frac{1}{R_{0}^{2}}\right) \delta r_{1}(t)+2 \int_{R}^{R_{1}} \frac{\delta r_{1}(\tau(x))}{x^{3}} d x .
$$

Imposing $\delta \sigma^{r r}(S(t), t)=-p_{i}(t)$ one obtains

$$
\left(\frac{1}{S^{2}(t)}-\frac{1}{R_{0}^{2}}\right) \delta r_{1}(t)-2 \int_{S(t)}^{R_{1}} \frac{\delta r_{1}(\tau(x))}{x^{3}} d x=\frac{p_{i}(t)}{2 \mu R_{1}}, \quad t>0 .
$$

Taking derivative with respect to $t$ of both sides one reduces the above integral equation to the following simple ODE:

$$
\frac{\dot{\delta r_{1}}}{\delta(t)}=\frac{R_{0}^{2}}{2 \mu R_{1}} \frac{S^{2}(t) \dot{p}_{i}(t)}{R_{0}^{2}-S^{2}(t)} .
$$

Integrating this ODE from 0 to $t$ and noting that $\delta r_{1}(0)=0$, one obtains

$$
\delta r\left(R_{1}, t\right)=\frac{R_{0}^{2}}{2 \mu R_{1}} \int_{0}^{t} \frac{S^{2}(\eta) \dot{p}_{i}(\eta)}{R_{0}^{2}-S^{2}(\eta)} d \eta .
$$


Therefore, substituting into (4.119), we have

$$
\delta r(R, t)-\delta \hat{r}(R)=\frac{R_{0}^{2}}{2 \mu R} \int_{\tau(R)}^{t} \frac{S^{2}(\eta) \dot{p}_{i}(\eta)}{R_{0}^{2}-S^{2}(\eta)} d \eta .
$$

Taking time derivative of both sides of $(4.124)_{1}$ we obtain

$$
\frac{\dot{\delta \sigma^{r r}}}{(R, t)}=-\left(\frac{R_{0}^{2}}{R^{2}}-1\right) \frac{S^{2}(t) \dot{p}_{i}(t)}{R_{0}^{2}-S^{2}(t)}
$$

Integrating the above relation from $\tau(R)$ to $t$ and noting that $\delta \sigma^{r r}(R, \tau(R))=-p_{i}(\tau(R))$, we have

$$
\delta \sigma^{r r}(R, t)=-p_{i}(\tau(R))-\left(\frac{R_{0}^{2}}{R^{2}}-1\right) \int_{\tau(R)}^{t} \frac{S^{2}(\eta) \dot{p}_{i}(\eta)}{R_{0}^{2}-S^{2}(\eta)} d \eta
$$

As regards the component $\delta \bar{\sigma}^{\theta \theta}(R, t)$, linearizing (4.30), valid in the case of inner accretion as well, we obtain

$$
\delta \bar{\sigma}^{\theta \theta}(R, t)=\delta \bar{\sigma}^{r r}(R, t)+\frac{4 \mu}{R}[\delta r(R, t)-\delta \bar{r}(R)]=\delta \bar{\sigma}^{r r}(R, t)+\frac{2 R_{0}^{2}}{R^{2}} \int_{\tau(R)}^{t} \frac{S^{2}(\eta) \dot{p}_{i}(\eta)}{R_{0}^{2}-S^{2}(\eta)} d \eta,
$$

which leads to

$$
\delta \bar{\sigma}^{\theta \theta}(R, t)=-p_{i}(\tau(R))+\left(\frac{R_{0}^{2}}{R^{2}}+1\right) \int_{\tau(R)}^{t} \frac{S^{2}(\eta) \dot{p}_{i}(\eta)}{R_{0}^{2}-S^{2}(\eta)} d \xi
$$

Residual stresses. Suppose surface growth stops at time $t_{a}$ and one removes the internal applied pressure. In the linear theory of accretion residual stresses can be calculated using superposition. For the cylinder of inner radius $S(t)$ and outer radius $R_{0}$ loaded by an internal pressure $-p_{i}\left(t_{a}\right)$ (unloading) and traction-free at the external radius one has

$$
\begin{aligned}
& U_{\text {unloading }}^{R}\left(R, t_{a}\right)=-\frac{1}{2} \frac{R_{0}^{2}}{R} \frac{S^{2}\left(t_{a}\right)}{R_{0}^{2}-S^{2}\left(t_{a}\right)} \frac{p_{i}\left(t_{a}\right)}{\mu}, \\
& \sigma_{\text {unloading }}^{R R}\left(R, t_{a}\right)=\frac{S^{2}\left(t_{a}\right)}{R^{2}} \frac{R_{0}^{2}-R^{2}}{R_{0}^{2}-S^{2}\left(t_{a}\right)} p_{i}\left(t_{a}\right), \\
& \bar{\sigma}_{\text {unloading }}^{\Theta \Theta}\left(R, t_{a}\right)=-\frac{S^{2}\left(t_{a}\right)}{R^{2}} \frac{R_{0}^{2}+R^{2}}{R_{0}^{2}-S^{2}\left(t_{a}\right)} p_{i}\left(t_{a}\right) .
\end{aligned}
$$

Adding the above to their corresponding values at the end of loading one obtains the residual displacement and the residual stresses as:

$$
\begin{aligned}
& U_{\text {res }}^{R}(R, t)=-\frac{R_{0}^{2} R}{R_{0}^{2}-R^{2}} \frac{p_{i}(\tau(R))}{2 \mu}+\frac{u_{1} R_{1}^{4}}{\mu R} \int_{\tau(R)}^{t_{a}} \frac{S(\eta) p_{i}(\eta)}{\left(R_{0}^{2}-S^{2}(\eta)\right)^{2}} d \eta \\
& \sigma_{\text {res }}^{R R}(R, t)=-2 u_{1} R_{0}^{2}\left(\frac{R_{0}^{2}}{R^{2}}-1\right) \int_{\tau(R)}^{t_{a}} \frac{S(\eta) p_{i}(\eta)}{\left(R_{0}^{2}-S^{2}(\eta)\right)^{2}} d \eta \\
& \bar{\sigma}_{\text {res }}^{\ominus \Theta}(R, t)=-\frac{2 R_{0}^{2}}{R_{0}^{2}-R^{2}} p_{i}(\tau(R))+2 u_{1} R_{0}^{2}\left(\frac{R_{1}^{2}}{R^{2}}+1\right) \int_{\tau(R)}^{t_{a}} \frac{S(\eta) p_{i}(\eta)}{\left(R_{0}^{2}-S^{2}(\eta)\right)^{2}} d \eta .
\end{aligned}
$$

\section{Surface Growth of a Thick Hollow Solid Sphere}

In this section we consider continuous accretion of a hollow spherical ball under a time-dependent internal pressure. We assume that the initial stress-free body is a hollow spherical ball with inner radius $R_{1}$ and outer radius $R_{0}$. At time $t$, under a specified internal pressure $p_{i}(t)$, the outer radius in the current configuration is $r_{2}(t)$. Note that we assume that the pressure $p_{i}(t)$ is apriori specified and the inner radius of the ball is part of the unknowns to be solved for. Similar to the cylinder problem in $\S 4$, we assume that accretion velocity is normal to the boundary with magnitude $u_{g}(t)$ in the current configuration. For a hollow ball we only consider 
outer accretion, i.e. material is continuously added on the outer boundary of the spherical ball. The metric of the Euclidean ambient space in spherical coordinates $(r, \theta, \phi)$ reads

$$
\mathbf{g}=\left(\begin{array}{ccc}
1 & 0 & 0 \\
0 & r^{2} & 0 \\
0 & 0 & r^{2} \sin ^{2} \theta
\end{array}\right)
$$

In the material manifold $\mathcal{B}$ we use the spherical coordinates $(R, \Theta, \Phi)$.

\subsection{Kinematics and material metric}

We assume motions of the form $(r, \theta, \phi)=(r(R, t), \Theta, \Phi)$, and hence the deformation gradient reads

$$
\mathbf{F}=\left(\begin{array}{ccc}
r^{\prime}(R, t) & 0 & 0 \\
0 & 1 & 0 \\
0 & 0 & 1
\end{array}\right)
$$

where $r^{\prime}(R, t)=\frac{\partial r(R, t)}{\partial R}$. Again we assign to each layer at radius $R$ in the material manifold a time of accretion $\tau(R)$. This map has the inverse $s=\tau^{-1}$, which assigns to each time the radius of the new spherical layer in the material manifold. It is assumed that $\tau\left(R_{0}\right)=0$ and $r_{2}(t)=r(s(t), t)$. Again we choose $U_{g}(t)=u_{g}(t)$ and assume that $u_{g}(t)=u_{0}>0$. We still have the relationships $r^{\prime}(s(t), t)=1$ and $s(t)=R_{0}+u_{0} t$.

For $R_{1} \leq R \leq R_{0}$ (the initial body), the material metric has the following representation in spherical coordinates.

$$
\mathbf{G}=\left(\begin{array}{ccc}
1 & 0 & 0 \\
0 & R^{2} & 0 \\
0 & 0 & R^{2} \sin ^{2} \Theta
\end{array}\right)
$$

Assuming an incompressible solid $J=r^{\prime} r^{2} / R^{2}=1$, one finds

$$
r^{3}(R, t)=r^{3}\left(R_{0}, t\right)+R^{3}-R_{0}^{3}, \quad R_{1} \leq R \leq R_{0} .
$$

The accreted shell at any instant of time $t$ is assumed to be stress free and hence $\mathbf{G}(R)=\varphi_{\tau(R)}^{*} \mathbf{g}(r(R, \tau(R)))$. In components, this reads $G_{A B}(s(t))=G_{A B}(R)=F^{a}{ }_{A}(R, \tau(R)) F_{B}^{b}(R, \tau(R)) g_{a b}(r(R, \tau(R)))$. Therefore, for $R_{0} \leq R \leq R_{0}+u_{0} t$ we have

$$
\mathbf{G}=\left(\begin{array}{ccc}
r^{\prime 2}(R, \tau(R)) & 0 & 0 \\
0 & r^{2}(R, \tau(R)) & 0 \\
0 & 0 & r^{2}(R, \tau(R)) \sin ^{2} \Theta
\end{array}\right)=\left(\begin{array}{ccc}
1 & 0 & 0 \\
0 & r^{2}(R, \tau(R)) & 0 \\
0 & 0 & r^{2}(R, \tau(R)) \sin ^{2} \Theta
\end{array}\right) .
$$

For the special case of $u_{g}(t)=u_{0}$, this is simplified to read

$$
\mathbf{G}=\left(\begin{array}{ccc}
1 & 0 & 0 \\
0 & r^{2}\left(R, \frac{R-R_{0}}{u_{0}}\right) & 0 \\
0 & 0 & r^{2}\left(R, \frac{R-R_{0}}{u_{0}}\right) \sin ^{2} \Theta
\end{array}\right) .
$$

Assuming that the accreted body is made of an incompressible solid we have

$$
r^{2}(R, t) r^{\prime}(R, t)=r^{2}\left(R, \frac{R-R_{0}}{u_{0}}\right) .
$$

The above equation can be integrated between $R_{0}$ and $R$ to give

$$
r^{3}(R, t)=r^{3}\left(R_{0}, t\right)+\int_{R_{0}}^{R} 3 r^{2}\left(x, \frac{x-R_{0}}{u_{0}}\right) d x, \quad R_{0} \leq R \leq R_{0}+u_{0} t
$$

from which, defining $r_{0}(t)=r\left(R_{0}, t\right)$ and $\bar{r}(R)=r(R, \tau(R))$, we obtain a decomposition analogous to (4.21):

$$
r^{3}(R, t)=r_{0}^{3}(t)+\int_{R_{0}}^{R} 3 \bar{r}^{2}(x) d x, \quad R_{0} \leq R \leq R_{0}+u_{0} t .
$$




\subsection{Calculation of stresses for an incompressible isotropic accreting spherical ball}

Next we calculate the stress field during the surface growth. For $R_{1} \leq R \leq R_{0}$, Finger tensor $\mathbf{b}^{\sharp}$ and $\mathbf{b}^{-1}$ are given by

$$
\mathbf{b}^{\sharp}=\left(\begin{array}{ccc}
\frac{R^{4}}{r^{4}(R, t)} & 0 & 0 \\
0 & \frac{1}{R^{2}} & 0 \\
0 & 0 & \frac{1}{R^{2} \sin ^{2} \Theta}
\end{array}\right), \quad \mathbf{b}^{-1}=\left(\begin{array}{ccc}
\frac{r^{4}(R, t)}{R^{4}} & 0 & 0 \\
0 & \frac{R^{2}}{r^{4}(R, t)} & 0 \\
0 & 0 & \frac{R^{2}}{r^{4}(R, t) \sin ^{2} \Theta}
\end{array}\right) .
$$

The principal stretches read

$$
I_{1}=\frac{R^{4}}{r^{4}(R, t)}+\frac{2 r^{2}(R, t)}{R^{2}}, \quad I_{2}=\frac{r^{4}(R, t)}{R^{4}}+\frac{2 R^{2}}{r^{2}(R, t)} .
$$

Therefore, the Cauchy stress has the following representation

$$
\boldsymbol{\sigma}=\left(\begin{array}{ccc}
-p+\frac{R^{4}}{r^{4}} \alpha+\frac{2 R^{2}}{r^{2}} \beta & 0 & 0 \\
0 & \frac{1}{r^{2}}\left[-p+\frac{r^{2}}{R^{2}} \alpha+\left(\frac{R^{2}}{r^{2}}+\frac{r^{4}}{R^{4}}\right) \beta\right] & 0 \\
0 & 0 & \frac{1}{r^{2} \sin ^{2} \Theta}\left[-p+\frac{r^{2}}{R^{2}} \alpha+\left(\frac{R^{2}}{r^{2}}+\frac{r^{4}}{R^{4}}\right) \beta\right]
\end{array}\right) .
$$

The non-trivial radial equilibrium equation is written as

$$
\frac{\partial \sigma^{r r}}{\partial r}+\frac{2}{r} \sigma^{r r}-r \sigma^{\theta \theta}-\left(r \sin ^{2} \theta\right) \sigma^{\phi \phi}=0 .
$$

This is simplified to read

$$
\frac{\partial \sigma^{r r}(R, t)}{\partial R}=\frac{2}{r(R, t)}\left[1-\frac{R^{6}}{r^{6}(R, t)}\right]\left[\alpha(R, t)+\frac{r^{2}(R, t)}{R^{2}} \beta(R, t)\right] .
$$

We know that $\sigma^{r r}\left(R_{1}, t\right)=-p_{i}(t)$, and hence, integrating the above ODE from $R_{1}$ to $R$, one obtains

$$
\sigma^{r r}(R, t)=-p_{i}(t)+\int_{R_{1}}^{R} \frac{2}{r(x, t)}\left[1-\frac{x^{6}}{r^{6}(x, t)}\right]\left[\alpha(x, t)+\frac{r^{2}(x, t)}{x^{2}} \beta(x, t)\right] d x .
$$

For $R_{0} \leq R \leq R_{0}+u_{0} t$, the Finger tensor $\mathbf{b}^{\sharp}$, and $\mathbf{b}^{-1}$ read

$$
\mathbf{b}^{\sharp}=\left(\begin{array}{ccc}
\frac{\bar{r}^{4}(R)}{r^{4}(R, t)} & 0 & 0 \\
0 & \frac{1}{\bar{r}^{2}(R)} & 0 \\
0 & 0 & \frac{1}{\bar{r}^{2}(R) \sin ^{2} \Theta}
\end{array}\right), \quad \mathbf{b}^{-1}=\left(\begin{array}{ccc}
\frac{r^{4}(R, t)}{\bar{r}^{4}(R)} & 0 & 0 \\
0 & \frac{\bar{r}^{2}(R)}{r^{4}(R, t)} & 0 \\
0 & 0 & \frac{\bar{r}^{2}(R)}{r^{4}(R, t) \sin ^{2} \Theta}
\end{array}\right) .
$$

The principal invariants of $\mathbf{b}$ are written as

$$
I_{1}=\frac{\bar{r}^{4}(R)}{r^{4}(R, t)}+2 \frac{r^{2}(R, t)}{\bar{r}^{2}(R)}, \quad I_{2}=\frac{r^{4}(R, t)}{\bar{r}^{4}(R)}+2 \frac{\bar{r}^{2}(R)}{r^{2}(R, t)} .
$$

The Cauchy stress in this region has the following representation

$$
\boldsymbol{\sigma}=\left(\begin{array}{ccc}
-p+\frac{\bar{r}^{4}}{r^{4}} \alpha+2 \frac{\bar{r}^{2}}{r^{2}} \beta & 0 & 0 \\
0 & \frac{1}{r^{2}}\left[-p+\frac{r^{2}}{\bar{r}^{2}} \alpha+\left(\frac{r^{4}}{\bar{r}^{4}}+\frac{\bar{r}^{2}}{r^{2}}\right) \beta\right] & 0 \\
0 & 0 & \frac{1}{r^{2} \sin ^{2} \Theta}\left[-p+\frac{r^{2}}{\bar{r}^{2}} \alpha+\left(\frac{r^{4}}{\bar{r}^{4}}+\frac{\bar{r}^{2}}{r^{2}}\right) \beta\right]
\end{array}\right) .
$$

Using the radial equilibrium equation, the radial Cauchy stress component is calculated as

$$
\begin{aligned}
\sigma^{r r}(R, t)= & \sigma^{r r}\left(R_{0}, t\right)+\int_{R_{0}}^{R} \frac{2}{r(x, t)}\left[1-\frac{\bar{r}^{6}(x)}{r^{6}(x, t)}\right]\left[\alpha(x, t)+\frac{r^{2}(x, t)}{\bar{r}^{2}(x)} \beta(x, t)\right] d x \\
= & -p_{i}(t)+\int_{R_{1}}^{R_{0}} \frac{2}{r(x, t)}\left[1-\frac{x^{6}}{r^{6}(x, t)}\right]\left[\alpha(x, t)+\frac{r^{2}(x, t)}{x^{2}} \beta(x, t)\right] d x \\
& +\int_{R_{0}}^{R} \frac{2}{r(x, t)}\left[1-\frac{\bar{r}^{6}(x)}{r^{6}(x, t)}\right]\left[\alpha(x, t)+\frac{r^{2}(x, t)}{\bar{r}^{2}(x)} \beta(x, t)\right] d x .
\end{aligned}
$$


We know that $\sigma^{r r}(s(t), t)=\sigma^{\theta \theta}(s(t), t)=0$. In particular, for the radial component, this gives

$$
\begin{aligned}
& \int_{R_{1}}^{R_{0}} \frac{2}{r(x, t)}\left[1-\frac{x^{6}}{r^{6}(x, t)}\right]\left[\alpha(x, t)+\frac{r^{2}(x, t)}{x^{2}} \beta(x, t)\right] d x \\
& +\int_{R_{0}}^{R_{0}+u_{0} t} \frac{2}{r(x, t)}\left[1-\frac{\bar{r}^{6}(x)}{r^{6}(x, t)}\right]\left[\alpha(x, t)+\frac{r^{2}(x, t)}{\bar{r}^{2}(x)} \beta(x, t)\right] d x=p_{i}(t) .
\end{aligned}
$$

Note that

$$
\bar{\sigma}^{\theta \theta}(R, t)=\bar{\sigma}^{\phi \phi}(R, t)=\sigma^{r r}(R, t)+\frac{r^{2}(R, t)}{\bar{r}^{2}(R)}\left[1-\frac{\bar{r}^{6}(R)}{r^{6}(R, t)}\right]\left[\alpha(R, t)+\frac{r^{2}(R, t)}{\bar{r}^{2}(R)} \beta(R, t)\right] .
$$

Therefore, $\bar{\sigma}^{\theta \theta}(s(t), t)=\bar{\sigma}^{\phi \phi}(R, t)=0$ are trivially satisfied. In the case of a homogeneous neo-Hookean solid, $\alpha(R, t)=\mu$ and $\beta(R, t)=0$. In this case, (5.20) is simplified to read

$$
\int_{R_{1}}^{R_{0}} \frac{2}{r(x, t)}\left[1-\frac{x^{6}}{r^{6}(x, t)}\right] d x+\int_{R_{0}}^{R_{0}+u_{0} t} \frac{2}{r(x, t)}\left[1-\frac{\bar{r}^{6}(x)}{r^{6}(x, t)}\right] d x=\frac{p_{i}(t)}{\mu} .
$$

One obtains the following problem in terms of the function $r(R, t)$ in the triangular region $\left\{(R, t) \in \mathbb{R}^{2} \mid R_{0} \leq\right.$ $\left.R \leq R_{0}+u_{0} t, t \in \mathbb{R}^{+}\right\}:$

$$
\left\{\begin{array}{l}
r^{2}(R, t) r^{\prime}(R, t)=r^{2}\left(R, \frac{R-R_{0}}{u_{0}}\right) \\
\int_{R_{1}}^{R_{0}} \frac{1}{\left(r^{3}\left(R_{0}, t\right)+R^{3}-R_{0}^{3}\right)^{\frac{1}{3}}}\left[1-\frac{x^{6}}{\left(r^{3}\left(R_{0}, t\right)+R^{3}-R_{0}^{3}\right)^{2}}\right] d x+\int_{R_{0}}^{R_{0}+u_{0} t} \frac{1-r^{\prime 3}(x, t)}{r(x, t)} d x=\frac{p_{i}(t)}{2 \mu}, \\
r\left(R_{0}, 0\right)=R_{0},
\end{array}\right.
$$

analogous to the one that was discussed in $\S 4$ for a cylindrical bar.

\subsection{Calculation of residual stresses for an incompressible isotropic accreting spher- ical ball}

Suppose that at some time $t=t_{a}$, the accretion process stops. The material metric has the following representation in spherical coordinates:

$$
\begin{aligned}
& R_{1} \leq R \leq R_{0}: \quad \mathbf{G}=\left(\begin{array}{ccc}
1 & 0 & 0 \\
0 & R^{2} & 0 \\
0 & 0 & R^{2} \sin ^{2} \Theta
\end{array}\right), \\
& R_{0} \leq R \leq R_{a}: \quad \mathbf{G}=\left(\begin{array}{ccc}
1 & 0 & 0 \\
0 & r^{2}\left(R, \frac{R-R_{0}}{u_{0}}\right) & 0 \\
0 & & r^{2}\left(R, \frac{R-R_{0}}{u_{0}}\right) \sin ^{2} \Theta
\end{array}\right) .
\end{aligned}
$$

Note that the function $r(R, t)$, and hence $r\left(R, \frac{R-R_{0}}{u_{0}}\right)$, has already been calculated. The motion from the material manifold to the residually-stressed configuration (under no applied loads) is denoted by $\tilde{\varphi}: \mathcal{B} \rightarrow$ $\mathcal{S}$, where in spherical coordinates we have: $\tilde{\varphi}(R, \Theta)=(\tilde{r}, \tilde{\theta}, \tilde{\phi})=(\tilde{r}(R), \Theta, \Phi)$. Using the incompressibility constraint one obtains

$$
\begin{array}{ll}
\tilde{r}^{3}(R)=\tilde{r}^{3}\left(R_{0}\right)+R^{3}-R_{0}^{3}, & R_{1} \leq R \leq R_{0}, \\
\tilde{r}^{3}(R)=\tilde{r}^{3}\left(R_{0}\right)+\int_{R_{0}}^{R} 3 r^{2}\left(x, \frac{x-R_{0}}{u_{0}}\right) d x, & R_{0} \leq R \leq R_{a},
\end{array}
$$


where $\tilde{r}\left(R_{0}\right)$ is an unknown that will be determined after enforcing the boundary and continuity conditions. In the absence of applied loads the boundary conditions read: $\tilde{\sigma}^{r r}\left(R_{1}\right)=\tilde{\sigma}^{r r}\left(R_{a}\right)=0$. One then has the following distribution of radial Cauchy stress (the continuity of traction $\tilde{\sigma}^{r r}\left(R_{0}^{-}\right)=\tilde{\sigma}^{r r}\left(R_{0}^{+}\right)$has been enfoced):

$$
\begin{aligned}
R_{1} \leq R \leq R_{0}: \quad \tilde{\sigma}^{r r}(R)= & \int_{R_{1}}^{R} \frac{2}{\tilde{r}(x)}\left[1-\frac{x^{6}}{\tilde{r}^{6}(x)}\right]\left[\alpha(x)+\frac{\tilde{r}^{2}(x)}{x^{2}} \beta(x)\right] d x, \\
R_{0} \leq R \leq R_{a}: \quad \tilde{\sigma}^{r r}(R)= & \int_{R_{1}}^{R_{0}} \frac{2}{\tilde{r}(x)}\left[1-\frac{x^{6}}{\tilde{r}^{6}(x)}\right]\left[\alpha(x)+\frac{\tilde{r}^{2}(x)}{x^{2}} \beta(x)\right] d x \\
& +\int_{R_{0}}^{R} \frac{2}{\tilde{r}(x)}\left[1-\frac{\bar{r}^{6}(x)}{\tilde{r}^{6}(x)}\right]\left[\alpha(x)+\frac{\tilde{r}^{2}(x)}{\bar{r}^{2}(x)} \beta(x)\right] d x .
\end{aligned}
$$

Similarly

$$
\begin{aligned}
& R_{1} \leq R \leq R_{0}: \quad \overline{\tilde{\sigma}}^{\theta \theta}(R)=\overline{\tilde{\sigma}}^{\phi \phi}(R)=\tilde{\sigma}^{r r}(R)+\frac{\tilde{r}^{2}(R)}{R^{2}}\left[1-\frac{R^{6}}{\tilde{r}^{6}(R)}\right]\left[\tilde{\alpha}(R)+\frac{\tilde{r}^{2}(R)}{R^{2}} \tilde{\beta}(R)\right], \\
& R_{0} \leq R \leq R_{a}: \quad \overline{\tilde{\sigma}}^{\theta \theta}(R)=\overline{\tilde{\sigma}}^{\phi \phi}(R)=\tilde{\sigma}^{r r}(R)+\frac{\tilde{r}^{2}(R)}{\bar{r}^{2}(R)}\left[1-\frac{\bar{r}^{6}(R)}{\tilde{r}^{6}(R)}\right]\left[\tilde{\alpha}(R)+\frac{\tilde{r}^{2}(R)}{\bar{r}^{2}(R)} \tilde{\beta}(R)\right] .
\end{aligned}
$$

The unknown constant $\tilde{r}\left(R_{0}\right)$ is determined using the condition $\tilde{\sigma}^{r r}\left(R_{a}\right)=0$, or

$$
\int_{R_{1}}^{R_{0}} \frac{2}{\tilde{r}(x)}\left[1-\frac{x^{6}}{\tilde{r}^{6}(x)}\right]\left[\alpha(x)+\frac{\tilde{r}^{2}(x)}{x^{2}} \beta(x)\right] d x+\int_{R_{0}}^{R_{a}} \frac{2}{\tilde{r}(x)}\left[1-\frac{\bar{r}^{6}(x)}{\tilde{r}^{6}(x)}\right]\left[\alpha(x)+\frac{\tilde{r}^{2}(x)}{\bar{r}^{2}(x)} \beta(x)\right] d x=0 .
$$

\subsection{Surface growth stresses in the linearized theory}

Linearization of the governing equations and stresses is similar to that of an infinite cylindrical bar. For $R_{1} \leq R \leq R_{0}$, for the perturbed motions we have $r_{\epsilon}^{3}(R, t)=r_{\epsilon}^{3}\left(R_{0}, t\right)+R^{3}-R_{2}^{3}$. Taking derivative with respect to $\epsilon$ on both sides and evaluating at $\epsilon=0$, one obtains

$$
\delta r(R, t)=\frac{R_{0}^{2}}{R^{2}} \delta r_{0}(t)
$$

with $\delta r_{0}(t)=\delta r\left(R_{0}, t\right)$. For $R_{0} \leq R \leq R_{0}+u_{0} t$

$$
r_{\epsilon}^{3}(R, t)=r_{\epsilon}^{3}\left(R_{0}, t\right)+\int_{R_{0}}^{R} 3 \bar{r}_{\epsilon}^{2}(x) d x .
$$

Again taking derivatives with respect to $\epsilon$ on both sides and evaluating at $\epsilon=0$, one obtains

$$
\delta r(R, t)=\frac{R_{0}^{2}}{R^{2}} \delta r_{0}(t)+\frac{1}{R^{2}} \int_{R_{0}}^{R} 2 x \delta \bar{r}(x) d x .
$$

Evaluating (5.31) at $t=\tau(R)$, one obtains

$$
\frac{1}{R} \int_{R_{0}}^{R} 2 x \delta \bar{r}(x) d x=\delta \bar{r}(R)-\frac{R_{0}^{2}}{R^{2}} \delta r\left(R_{0}, \tau(R)\right) .
$$

Substituting the above relation back into (5.31), one has

$$
\delta r(R, t)-\delta \bar{r}(R)=\frac{R_{0}^{2}}{R^{2}}\left[\delta r\left(R_{0}, t\right)-\delta r\left(R_{0}, \tau(R)\right)\right] .
$$

For $R_{1} \leq R \leq R_{0}$, the linearized radial Cauchy stress reads

$$
\delta \sigma^{r r}(R, t)=-p_{i}(t)+12 \mu \int_{R_{1}}^{R} \frac{\delta r(x, t)}{x^{2}} d x=-p_{i}(t)+4 \mu R_{0}^{2}\left(\frac{1}{R_{1}^{3}}-\frac{1}{R^{3}}\right) \delta r_{0}(t),
$$


where we used (5.29). For $R_{0} \leq R \leq s(t)$ we have

$$
\delta \sigma^{r r}(R, t)=-p_{i}(t)+4 \mu R_{0}^{2}\left(\frac{1}{R_{1}^{3}}-\frac{1}{R_{0}^{3}}\right) \delta r_{0}(t)+12 \mu \int_{R_{0}}^{R} \frac{\delta r(x, t)-\delta \bar{r}(x)}{x^{2}} d x .
$$

Using (5.33) we obtain

$$
\delta \sigma^{r r}(R, t)=-p_{i}(t)+4 \mu R_{0}^{2}\left(\frac{1}{R_{1}^{3}}-\frac{1}{R^{3}}\right) \delta r_{0}(t)-3 \int_{R_{0}}^{R} \frac{\delta \delta r_{0}(\tau(x))}{x^{4}} d x .
$$

We know that $\sigma_{\epsilon}^{r r}(s(t), t)=0$ and hence $\delta \sigma^{r r}(s(t), t)=0$. Therefore

$$
\left(\frac{1}{R_{1}^{3}}-\frac{1}{s^{3}(t)}\right) \delta r_{0}(t)-3 \int_{R_{0}}^{R_{0}+u_{0} t} \frac{\delta \delta r_{0}(\tau(x))}{x^{4}} d x=\frac{p_{i}(t)}{4 \mu R_{0}^{2}}, \quad t>0 .
$$

Taking derivative with respect to $t$ of both sides one reduces the above integral equation to the following simple ODE:

$$
\frac{\dot{\delta r_{0}}}{\delta(t)}=\frac{R_{1}^{3}}{4 \mu R_{0}} \frac{s^{3}(t) \dot{p}_{i}(t)}{s^{3}(t)-R_{1}^{3}} .
$$

Integrating this ODE from 0 to $t$ and noting that $\dot{\delta r}\left(R_{0}, 0\right)=0$, one obtains

$$
\delta r_{0}(t)=\frac{R_{1}^{3}}{4 \mu R_{0}^{2}} \int_{0}^{t} \frac{s^{3}(\eta) \dot{p}_{i}(\eta)}{s^{3}(\eta)-R_{1}^{3}} d \eta
$$

Therefore, Eq. (5.33) is simplified to read

$$
\delta r(R, t)-\delta \bar{r}(R)=\frac{R_{1}^{3}}{4 \mu R^{2}} \int_{\tau R}^{t} \frac{s^{3}(\eta) \dot{p}_{i}(\eta)}{s^{3}(\eta)-R_{1}^{3}} d \eta,
$$

which, after solving (5.32) for $\delta \bar{r}(R)$, allows one to find $\delta r(R, t)$. Taking time derivative of both sides of $(5.36)$ we obtain

$$
\dot{\overline{\delta \sigma^{r r}}}(R, t)=-\dot{p}_{i}(t)+4 \mu R_{0}^{2}\left(\frac{1}{R_{1}^{3}}-\frac{1}{R^{3}}\right) \dot{\overline{\delta r}}\left(R_{0}, t\right) .
$$

Integrating the above relation from $\tau(R)$ to $t$ and noting that $\delta \sigma^{r r}(R, \tau(R))=0$, we have

$$
\delta \sigma^{r r}(R, t)=-p_{i}(t)+p_{i}(\tau(R))+\left(1-\frac{R_{1}^{3}}{R^{3}}\right) \int_{\tau(R)}^{t} \frac{s^{3}(\eta) \dot{p}_{i}(\eta)}{s^{3}(\eta)-R_{1}^{3}} d \eta .
$$

Similarly, the other two non-zero components of the Cauchy stress have the following linearized expressions

$$
\delta \bar{\sigma}^{\theta \theta}(R, t)=\delta \bar{\sigma}^{\phi \phi}(R, t)=-p_{i}(t)+p_{i}(\tau(R))+\left(1+\frac{R_{1}^{3}}{2 R^{3}}\right) \int_{\tau(R)}^{t} \frac{s^{3}(\eta) \dot{p}_{i}(\eta)}{s^{3}(\eta)-R_{1}^{3}} d \eta
$$

Residual stresses. We follow the same approach that was used for the cylinder examples. The unloading fields are

$$
\begin{aligned}
& U_{\text {unloading }}^{R}\left(R, t_{a}\right)=-\frac{R_{1}^{3}}{R^{2}} \frac{s 32\left(t_{a}\right)}{s^{3}\left(t_{a}\right)-R_{1}^{3}} \frac{p_{i}\left(t_{a}\right)}{4 \mu}, \\
& \sigma_{\text {unloading }}^{R R}\left(R, t_{a}\right)=\frac{R_{1}^{3}}{R^{3}} \frac{s^{3}\left(t_{a}\right)-R^{3}}{s^{3}\left(t_{a}\right)-R_{1}^{3}} p_{i}\left(t_{a}\right), \\
& \bar{\sigma}_{\text {unloading }}^{\Theta \Theta}\left(R, t_{a}\right)=-\frac{R_{1}^{3}}{R^{3}} \frac{\frac{1}{2} s^{3}\left(t_{a}\right)+R^{3}}{s^{3}\left(t_{a}\right)-R_{1}^{3}} p_{i}\left(t_{a}\right) .
\end{aligned}
$$


Integrating Eqs. (5.40), (5.42) and (5.43) by parts, and adding (5.44) we obtain the following residual fields:

$$
\begin{gathered}
U_{\text {res }}^{R}\left(R, t_{a}\right)=-\frac{R_{1}^{3} R}{R^{3}-R_{1}^{3}} \frac{p_{i}\left(\frac{R-R_{0}}{u_{0}}\right)}{4 \mu}+\frac{3 u_{0} R_{1}^{6}}{4 \mu R^{2}} \int_{\frac{R-R_{0}}{u_{0}}}^{t_{a}} \frac{s^{2}(\eta) p_{i}(\eta)}{\left(s^{3}(\eta)-R_{1}^{3}\right)^{2}} d \eta, \\
\sigma_{\text {res }}^{R R}\left(R, t_{a}\right)=3 u_{0} R_{1}^{3}\left(1-\frac{R_{1}^{3}}{R^{3}}\right) \int_{\frac{R-R_{0}}{u_{0}}}^{t_{a}} \frac{s^{2}(\eta) p_{i}(\eta)}{\left(s^{3}(\eta)-R_{1}^{3}\right)^{2}} d \eta, \\
\delta \bar{\sigma}_{\text {res }}^{\theta \theta}(R, t)=\delta \bar{\sigma}_{\text {res }}^{\phi \phi}(R, t)=-\frac{3}{2} \frac{R_{1}^{3}}{R^{3}-R_{1}^{3}} p_{i}\left(\frac{R-R_{0}}{u_{0}}\right)+3 u_{0} R_{1}^{3}\left(1+\frac{R_{1}^{3}}{2 R^{3}}\right) \int_{\frac{R-R_{0}}{u_{0}}}^{t_{a}} \frac{s^{2}(\eta) p_{i}(\eta)}{\left(s^{3}(\eta)-R_{1}^{3}\right)^{2}} d \eta .
\end{gathered}
$$

\section{$6 \quad$ Numerical Examples}

In this section we present some numerical examples of surface growth for cylindrical and spherical bodies made of neo-Hookean solids.

\subsection{Numerical solution of the governing equations}

We introduce an auxiliary problem to (4.33) in which the original cylinder is the result of accretion "from nothing" with no internal pressure. The auxiliary problems of inner accretion of a cylindrical bar and outer accretion of a spherical ball are similar. The domain of the auxiliary problem is the region $R_{1} \leq R \leq R_{0}+u_{0} t$, and has its starting time at $t^{*}=-\frac{R_{0}-R_{1}}{u_{0}}$. Now we show that this approach is equivalent to the problem (4.33).

For $t^{*} \leq t \leq 0$ the governing equations (4.33) read

$$
\begin{cases}r(R, t) r^{\prime}(R, t)=r\left(R, \frac{R-R_{0}}{u_{0}}\right) & \text { for } R_{1} \leq R \leq R_{0}+u_{0} t, t^{*} \leq t \leq 0, \\ \int_{R_{0}}^{R_{0}+u_{0} t} \frac{1}{\bar{r}(x)}\left[1-\frac{\bar{r}^{4}(x)}{r^{4}(x, t)}\right] d x=0 & \text { for } t^{*} \leq t \leq 0, \\ r\left(R_{1}, 0\right)=R_{1} . & \end{cases}
$$

It is straightforward to check that $r(R, t)=R$ is a solution of this problem. This means that at time $t=0$ we obtain the stress-free cylinder of internal radius $R_{1}$ and external radius $R_{0}$. For $t \geq 0$ the problem becomes

$$
\begin{cases}r(R, t) r^{\prime}(R, t)=r\left(R, \frac{R-R_{0}}{u_{0}}\right) & \text { for } R_{1} \leq R \leq R_{0}+u_{0} t \\ \int_{R_{1}}^{R_{0}+u_{0} t} \frac{1}{\bar{r}(x)}\left[1-\frac{\bar{r}^{4}(x)}{r^{4}(x, t)}\right] d x=\frac{p_{i}(t)}{\mu} & \text { for } t \geq 0 \\ r\left(R_{1}, 0\right)=R_{1} & \end{cases}
$$

where the integral can be rewritten as

$$
\int_{R_{1}}^{R_{0}} \frac{1}{\bar{r}(x)}\left[1-\frac{\bar{r}^{4}(x)}{r^{4}(x, t)}\right] d x+\int_{R_{0}}^{R_{0}+u_{0} t} \frac{1}{\bar{r}(x)}\left[1-\frac{\bar{r}^{4}(x)}{r^{4}(x, t)}\right] d x .
$$

Note that in the region $R_{1} \leq R \leq R_{0}$, since $t^{*} \leq \frac{R-R_{0}}{u_{0}} \leq 0$, we simply have $\bar{r}(R)=r\left(R, \frac{R-R_{0}}{u_{0}}\right)=R$. Thus, we obtain the following two problems

$$
\begin{cases}r(R, t) r^{\prime}(R, t)=R & \text { for } R_{1} \leq R \leq R_{0}, \\ \int_{R_{1}}^{R_{0}} \frac{1}{\bar{r}(x)}\left[1-\frac{\bar{r}^{4}(x)}{r^{4}(x, t)}\right] d x=\frac{p_{i}(t)}{\mu} & \text { for } t \geq 0, \\ r(R, 0)=R, & \end{cases}
$$


and

$$
\begin{cases}r(R, t) r^{\prime}(R, t)=r\left(R, \frac{R-R_{0}}{u_{0}}\right) & \text { for } R_{0} \leq R \leq R_{0}+u_{0} t \\ \int_{R_{1}}^{R_{0}} \frac{1}{x}\left[1-\frac{x^{4}}{r^{4}(x, t)}\right] d x+\int_{R_{0}}^{R_{0}+u_{0} t} \frac{1}{\bar{r}(x)}\left[1-\frac{\bar{r}^{4}(x)}{r^{4}(x, t)}\right] d x=\frac{p_{i}(t)}{\mu} & \text { for } t \geq 0 \\ r\left(R_{0}, 0\right)=R_{0}, & \end{cases}
$$

which is consistent with (4.18) for the initial body and is identical to (4.33) for the secondary body. Therefore, considering a single domain $R_{1} \leq R \leq R_{0}+u_{0} t$ starting at time $t^{*}$ with $p_{i}=0$ for $t^{*} \leq t \leq 0$ is equivalent to solving the governing equations in two different domains starting at time 0 . We solve the auxiliary problem in our numerical simulations, as it is numerically more convenient. For the problem (6.5) we use finite differences, while the nonlinear equations, including (4.53) for the residually-stressed configuration, are solved using the Newton-Raphson method.

\subsection{Numerical results}

In the following examples we normalize stresses by the shear modulus $\mu$ and work with the dimensionless time $t / t_{a}$ and the dimensionless radial coordinate $R / R_{1}$. We also assume that $R_{0} / R_{1}=1.25$ and $R_{a} / R_{1}=2.25$, which means that $u_{0}=R_{1} / t_{a}=1$.

(a)

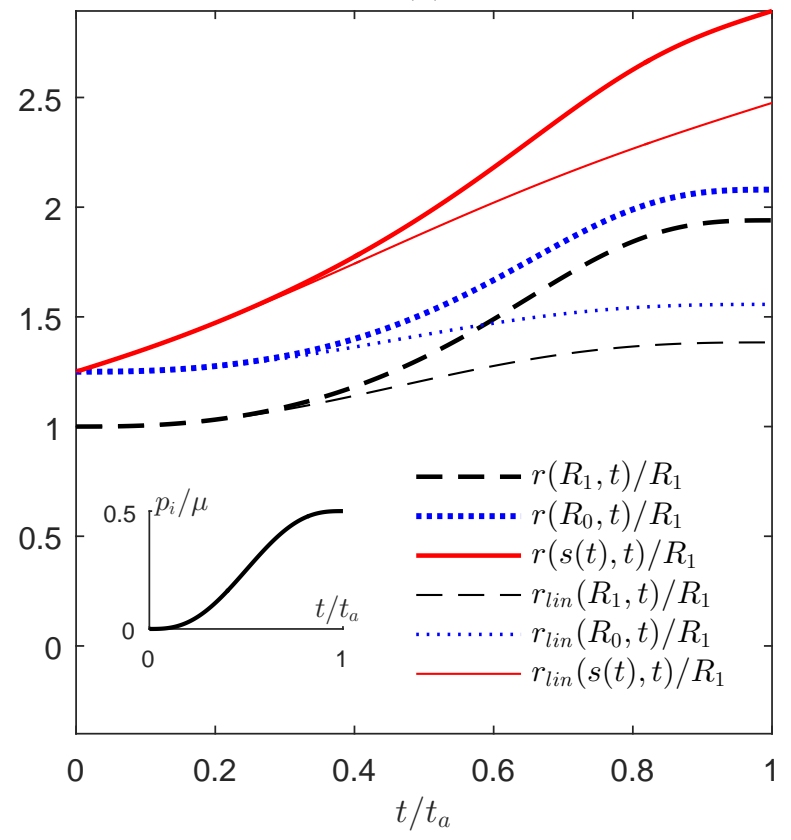

(b)

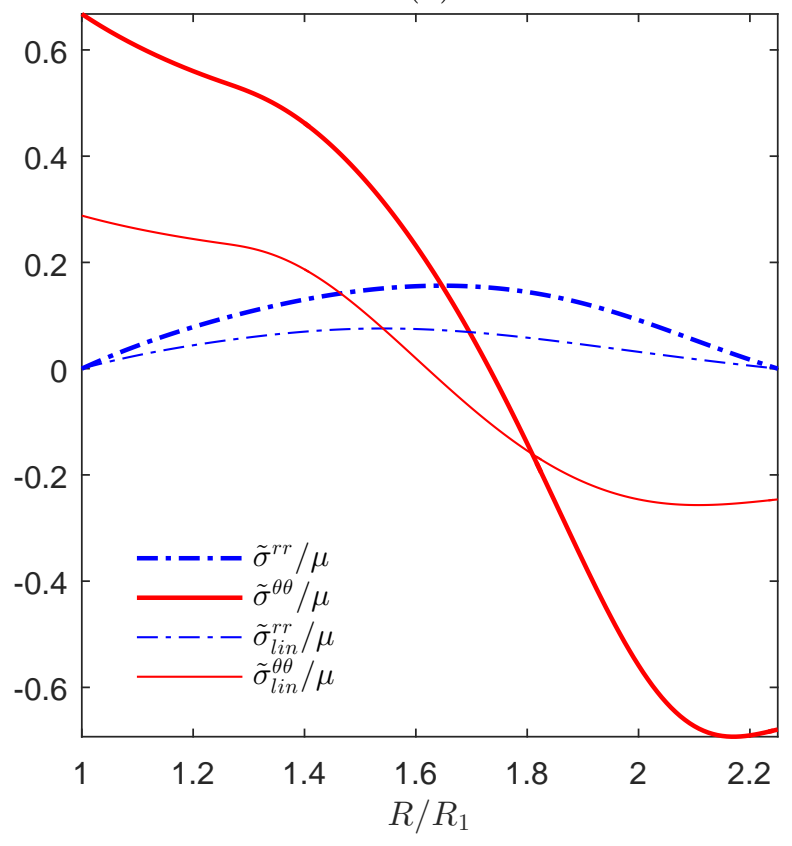

Figure 5: Outer surface growth under a monotonically increasing internal pressure $p_{i}(t)$. (a) The inner, interface, and outer radii and their linear counterparts as functions of time. (b) The radial and circumferential residual stresses and their linear counterparts.

Example 1. Fig.5(a) shows the time dependency of the radii of the inner and outer boundaries of a cylinder under outer accretion and also that of the interface between the initial and the secondary bodies in the current configuration, and their linear counterparts. The cylinder is under the monotonically-increasing pressure shown in the figure. It is seen that for $t>0.5 t_{a}$ the nonlinear effects are quite noticeable. Fig.5(b) shows the profiles of the radial and circumferential residual stresses and their linear counterparts. It is seen that the nonlinear circumferential stress is more than twice as much as the linear one at some points. Note that the linear and nonlinear stresses do not attain their minima and maxima at the same points. Also, note that as was explained 
(a)

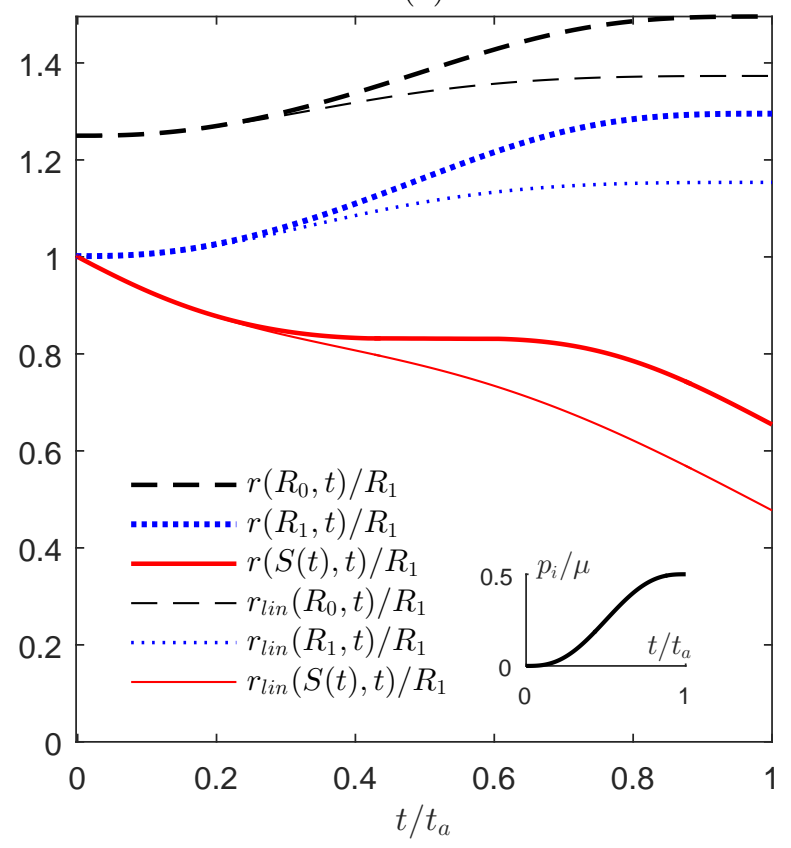

(b)

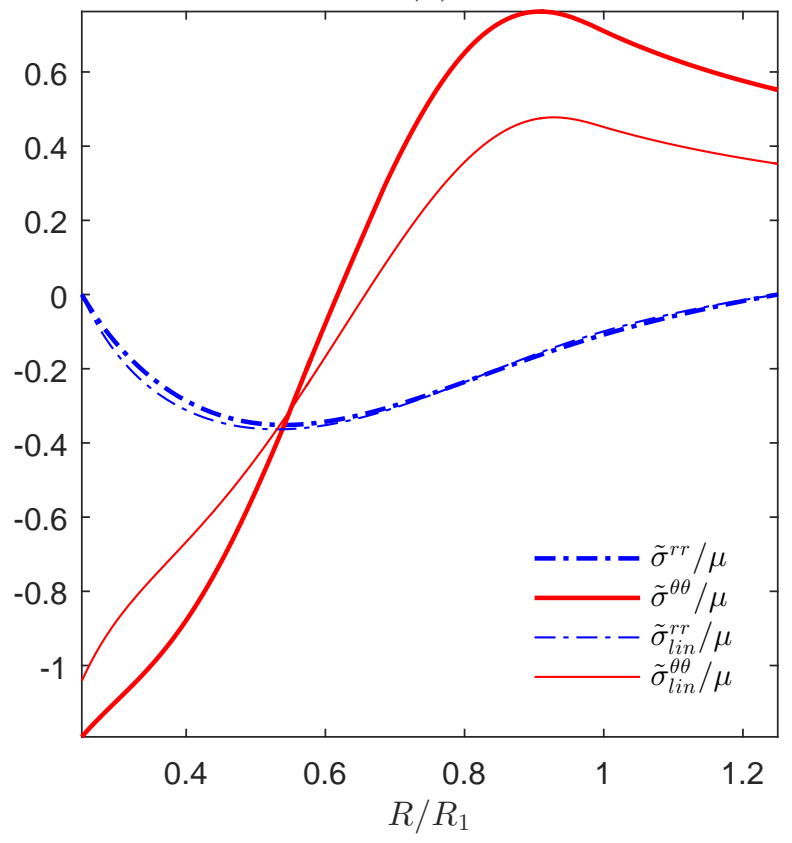

Figure 6: Inner surface growth under a monotonically increasing internal pressure $p_{i}(t)$. (a) The outer, interface, and inner radii and their linear counterparts as functions of time. (b) The radial and circumferential residual stresses and their linear counterparts.

in Remark 4.5, in the initial body, i.e. for $1 \leq R / R_{1} \leq 1.25, \tilde{\sigma}^{r r}$ is strictly increasing while $\overline{\tilde{\sigma}}^{\theta \theta}$ is strictly decreasing. Note also that the circumferential component of the residual Cauchy stress changes sign along the thickness of the cylinder. Fig.6 shows the same quantities for the inner accretion of the cylinder. We observe the same change of sign of the circumferential component of the Cauchy stress. For the outer accretion of a spherical ball, Fig. 7 shows the same quantities that were discussed for the cylinder examples.

Example 2. In Fig. 8 we compare two outer accreted cylinders that are manufactured under cycles of internal pressure or suction. It is seen that the nonlinear deformation and residual stress fields in the two cylinders are quite different. Note also that under internal pressure the nonlinear stresses and radial displacements are larger (in absolute value) than their linear counterparts, while under an internal suction the linear quantities are larger. However, as expected, the linear displacement and residual stress fields are the same up to a sign in the two cases.

Example 3. Next, in Fig.9, we consider two cylinders that have identical dimensions. One (outer accreted cylinder) is built in an additive manufacturing process starting with an initial tube. The other one (nonaccreted cylinder) has no residual stresses. For the outer accreted cylinder we consider two cases: one additively manufactured under an internal pressure and one manufactured under an internal suction. The maximum value of pressure or suction is $0.1 \mu$ as shown in Fig.9. Each cylinder is loaded under an internal pressure or suction of magnitude $p_{e}=0.3 \mu$. The radial stress $\sigma^{r r}$ does not seem to change much with or without surface growth. The difference is evident especially for $\bar{\sigma}^{\theta \theta}$. In Fig.10 we make the same comparison for inner accretion, obtaining similar results. These observations indicate that stress distribution under service loads may be reduced by controlling the residual stress distribution in an additively-manufactured cylindrical bar.

Example 4. In Fig.11 the stiffness of an inner accreted cylinder is compared with that of its corresponding non-accreted cylinder. We consider two cases: accretion under an internal pressure and accretion under an internal suction. The radial displacement of the inner boundary is calculated with respect to the position that corresponds to $p_{e}=0$, which is $R_{a}$ for the non-accreted cylinder and $\tilde{r}\left(R_{a}\right)$ for the accreted one. Displacements are normalized using $R_{1}$. Note that when $p_{i}$ and $p_{e}$ have opposite signs the accreted cylinder is stiffer than the 
(a)

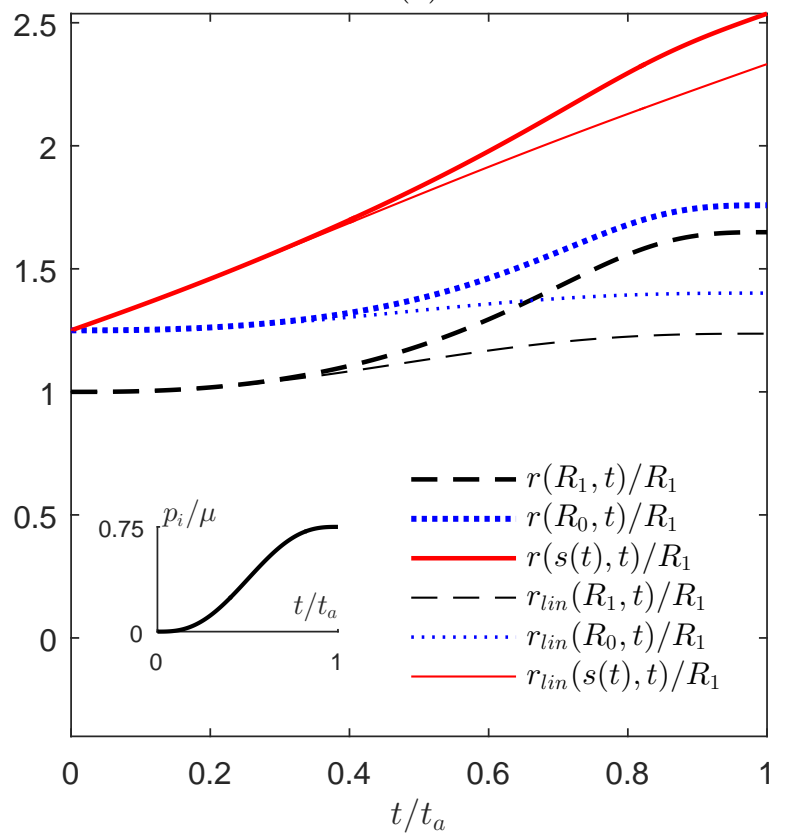

(b)

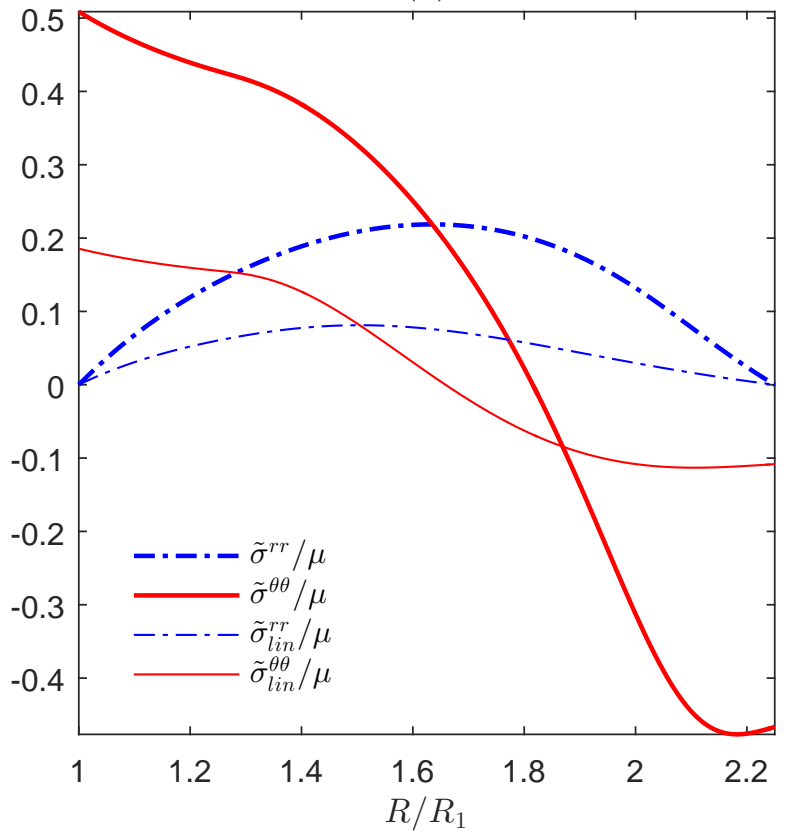

Figure 7: Surface growth of a spherical ball under a monotonically increasing internal pressure $p_{i}(t)$. (a) The inner, interface, and outer radii and their linear counterparts as functions of time. (b) The radial and circumferential residual stresses and their linear counterparts.

non-accreted one. This observation suggests that the stiffness of an additively manufactured structure can be controlled by controlling the surface growth process.

Example 5. In Fig.12 the history of the inner radius of a cylinder during an outer surface growth under two different loading cycles is shown $\left(R_{0} / R_{1}=1.25\right.$ and $\left.R_{a} / R_{1}=2.25\right)$. It is seen that in both the nonlinear and linear solutions the final radius is different from its initial value. This is due to the anelastic nature of surface growth even for a hyper-elastic solid.

\section{Conclusions}

In this paper we formulated the initial-boundary value problems of surface growth for cylindrical and spherical bodies made of nonlinear elastic solids. To simplify the calculations we restricted ourselves to incompressible and isotropic materials. As model problems we considered infinitely-long hollow cylindrical bars and hollow spherical balls. While a cylindrical body is under a time-dependent internal pressure (or suction) material is continuously added to either its outer or inner boundary. For a hollow spherical ball stress-free material is added to its outer boundary. Our goal was to calculate the time-dependent stress field during accretion and the residual stress field after the completion of accretion. We solved these problems in the framework of geometric anelasticity. We first constructed a family of Riemannian material manifolds. The growing body is stress-free at any instant of time in its material manifold. We calculated the material metric and observed that it depends on the history of loading and deformation during surface growth and the accretion velocity. Having a material manifold the surface growth problem is transformed into a classical nonlinear elasticity problem with an evolving reference configuration. We showed that calculation of accretion stresses for an incompressible solid is reduced to solving a nonlinear partial integral equation. We then linearized the nonlinear governing equations about a stress-free equilibrium configuration and showed that the resulting linear partial integral equation gives displacements and stresses that are identical to those calculated using the linear theory of accretion of Brown and Goodman [1963]. The governing equations were solved numerically in the case of a neo-Hookean solid. We also calculated the residual stresses. 
(a)

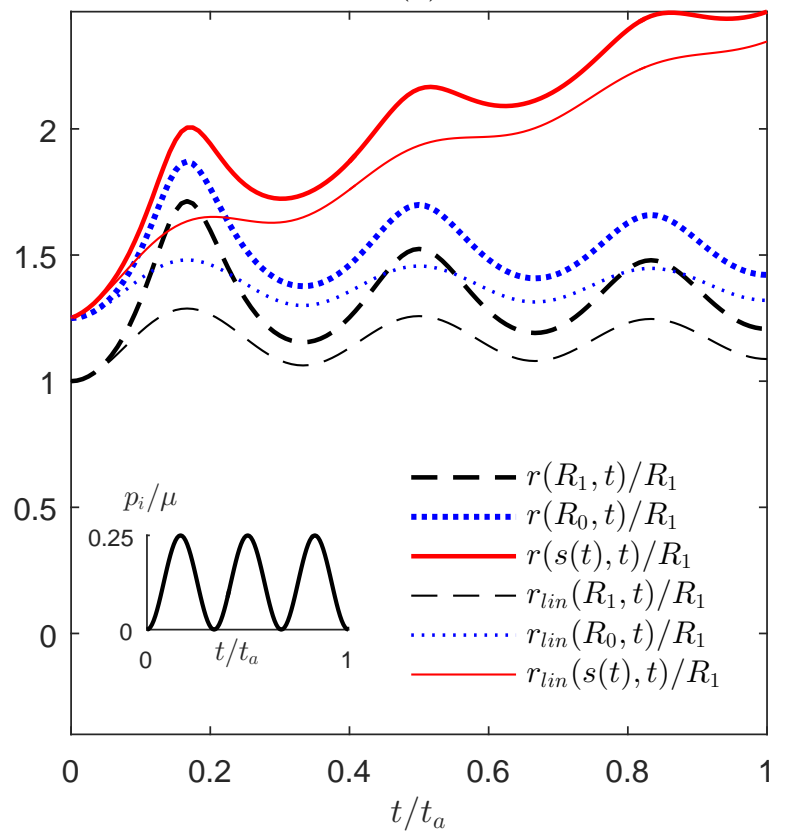

(c)

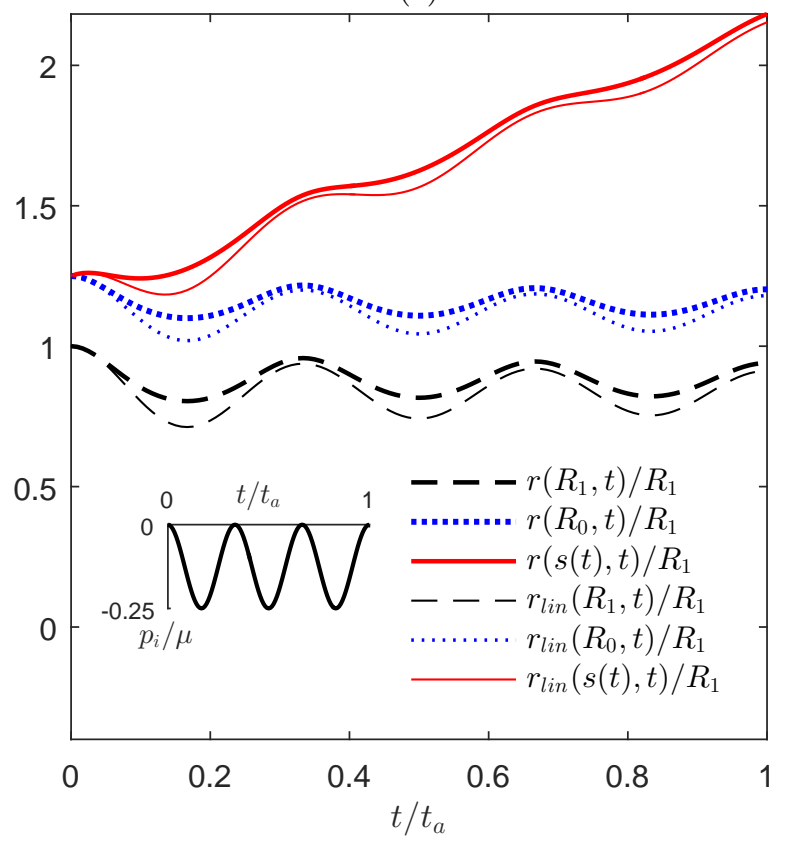

(b)

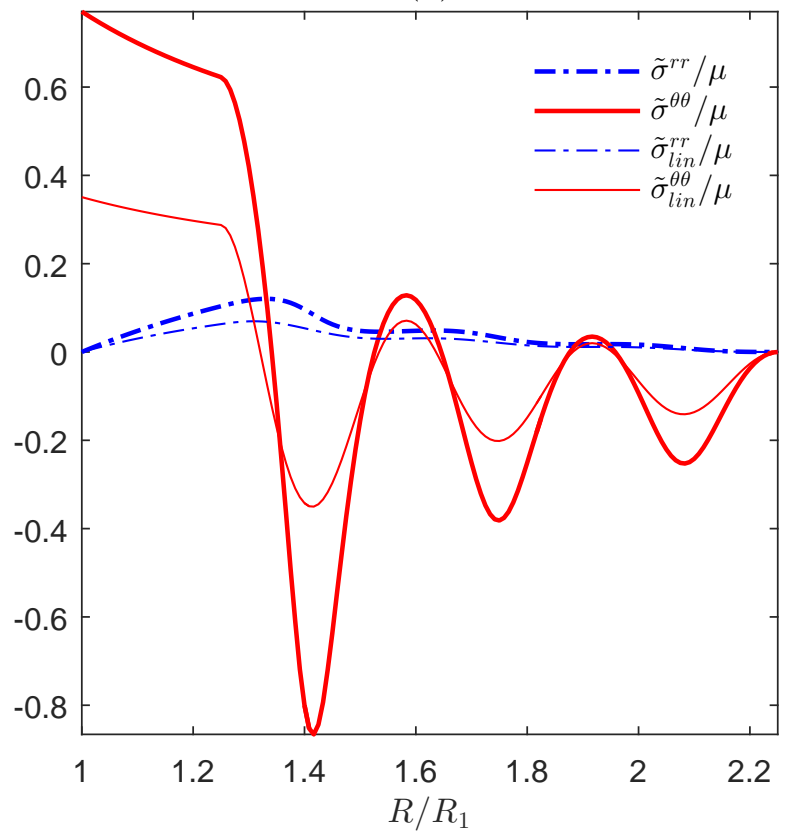

(d)

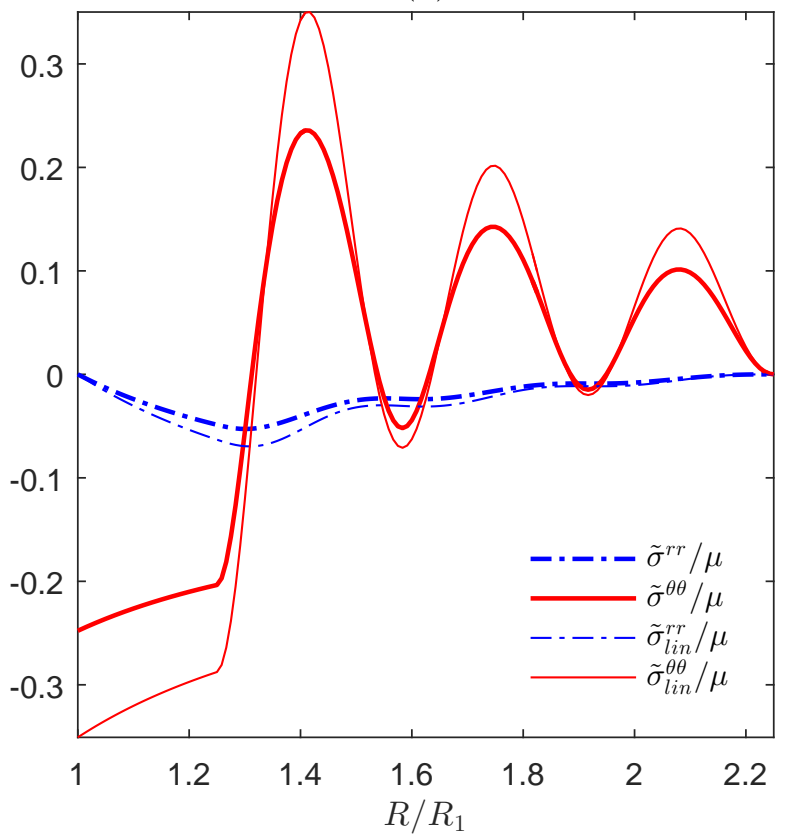

Figure 8: Outer surface growth under internal pressure ((a), (b)) or internal suction ((c), (d)) cycles. (a) and (c) show the inner, interface and outer radii and their linear counterparts as functions of time. (b) and (d) show the radial and circumferential residual stresses and their linear counterparts.

Extending the present theory to non-normal surface growth of arbitrary-shaped bodies will be the subject of a future communication. Another extension of the present work would be modeling surface growth in thin shells. Recently, Sadik et al. [2016] formulated a nonlinear shell theory with bulk growth. Kinematics of a shell is described by the first and the second fundamental forms that evolve in the material manifold as a result of bulk growth. It would be interesting to see how the fundamental forms of an accreting shell depend on the history of loading and deformation during surface growth. Other interesting problems for future work would be taking into 
(a)

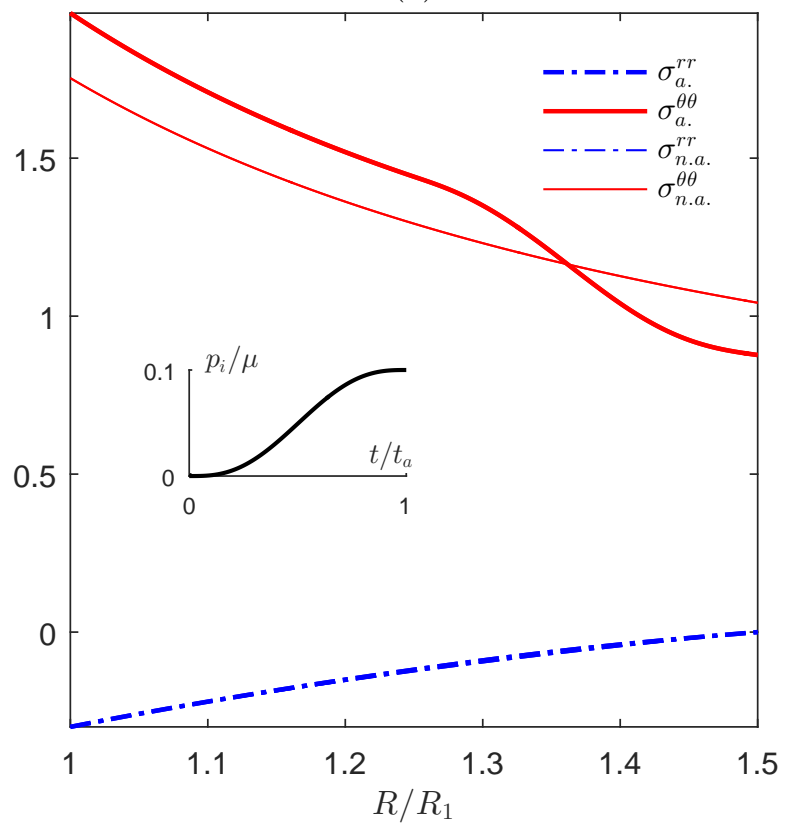

(c)

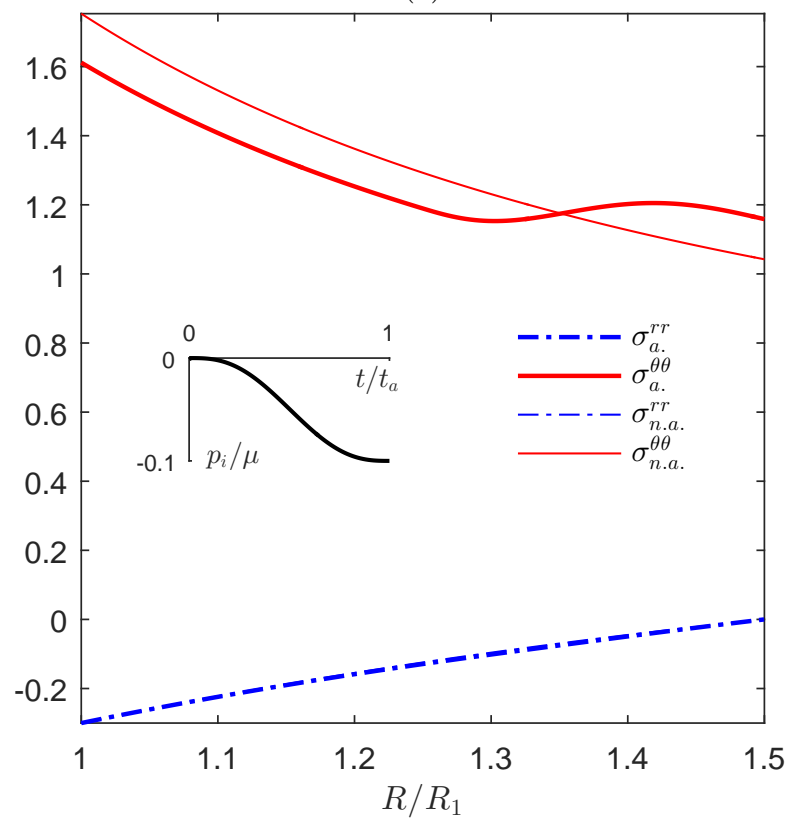

(b)

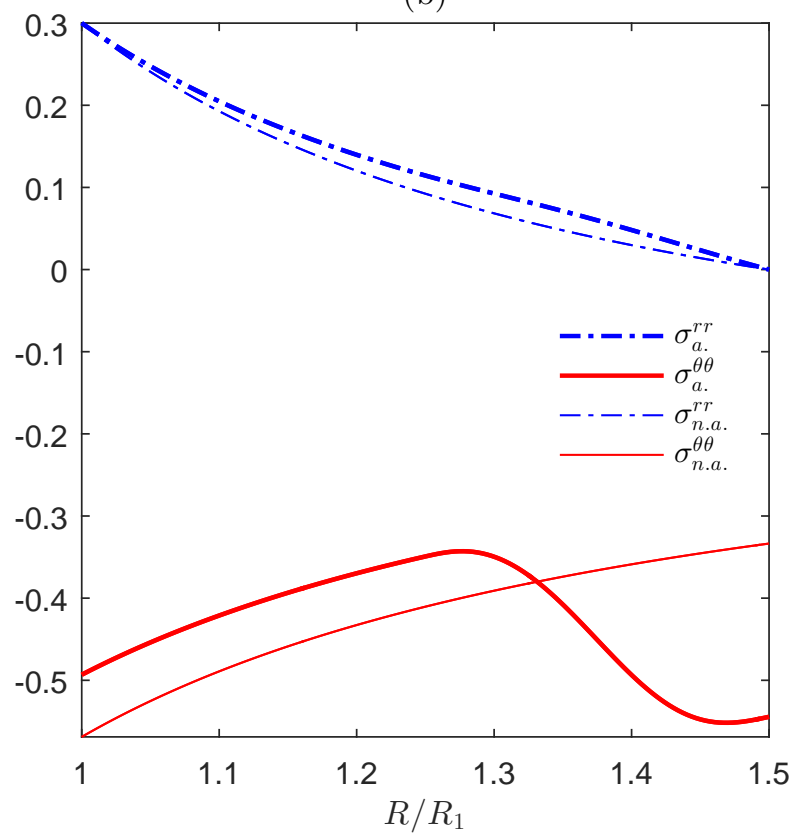

(d)

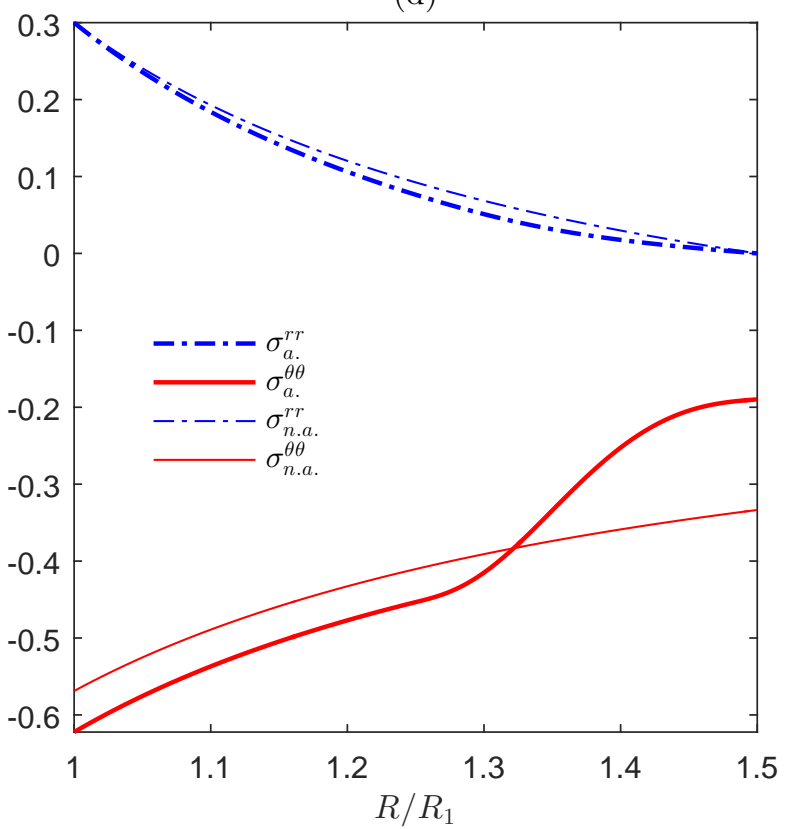

Figure 9: Comparison between an accreted and a non-accreted cylinder both with inner and outer radii $R_{1}$ and $R_{a}$, respectively, loaded by an internal pressure or suction $p_{e} / \mu=0.3$. The outer accreted cylinder was loaded during the accretion process by $p_{i}(t)$. (a) $p_{i}(t)>0$ and $p_{e}>0$, (b) $p_{i}(t)>0$ and $p_{e}<0$, (c) $p_{i}(t)<0$ and $p_{e}>0$, (d) $p_{i}(t)<0$ and $p_{e}<0$.

account material anisotropies and compressibility, surface growth under twist or uni-axial tension/compression, and the effect of pre-stress of the accreting material on the stiffness of an additively-manufactured structure.

The present nonlinear theory of surface growth can potentially be used for optimizing an additive manufacturing process for achieving best stiffness and smallest stresses under service loads of the structure. Knowing the service loads one may reduce the stresses by controlling the residual stresses. The factors that affect the residual stress distribution are: loading during surface growth, speed of adding material, and the state of stress of the added material at the time of attachment to the structure. The nonlinear surface growth theory presented 
(a)

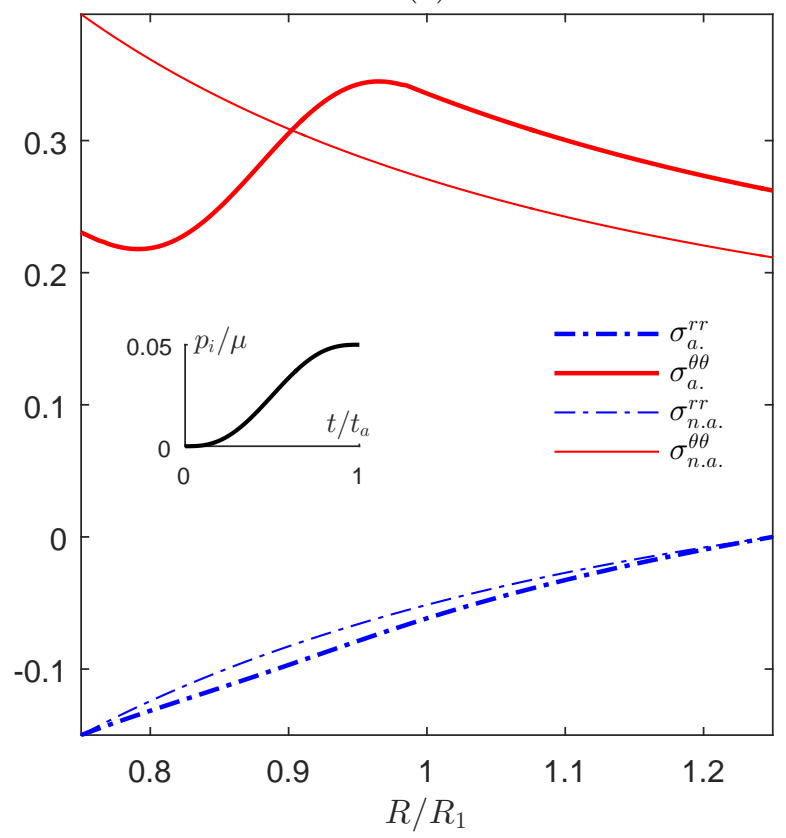

(c)

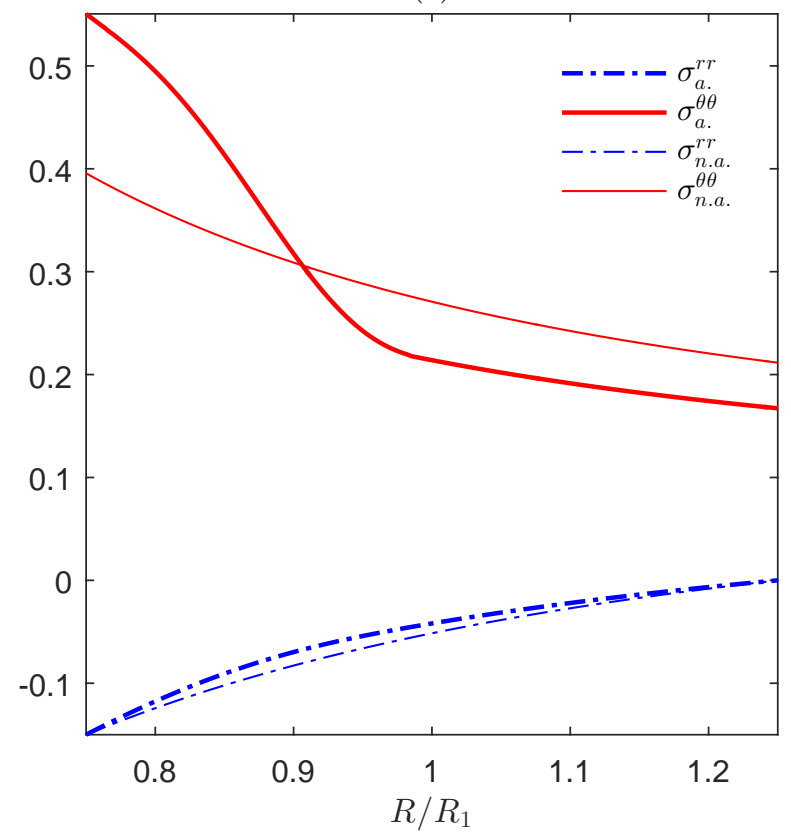

(b)

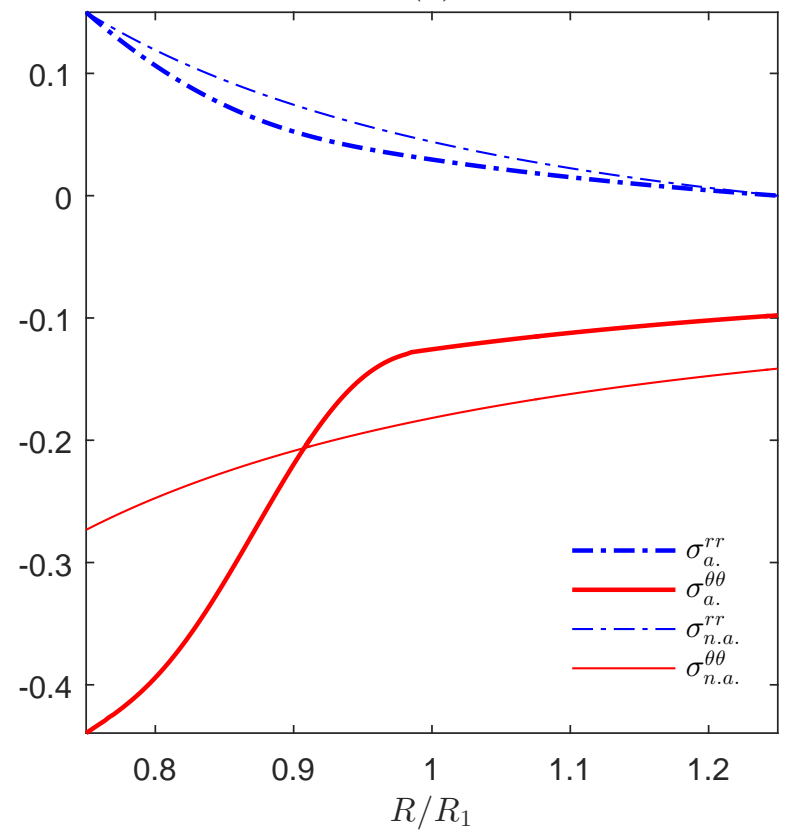

(d)

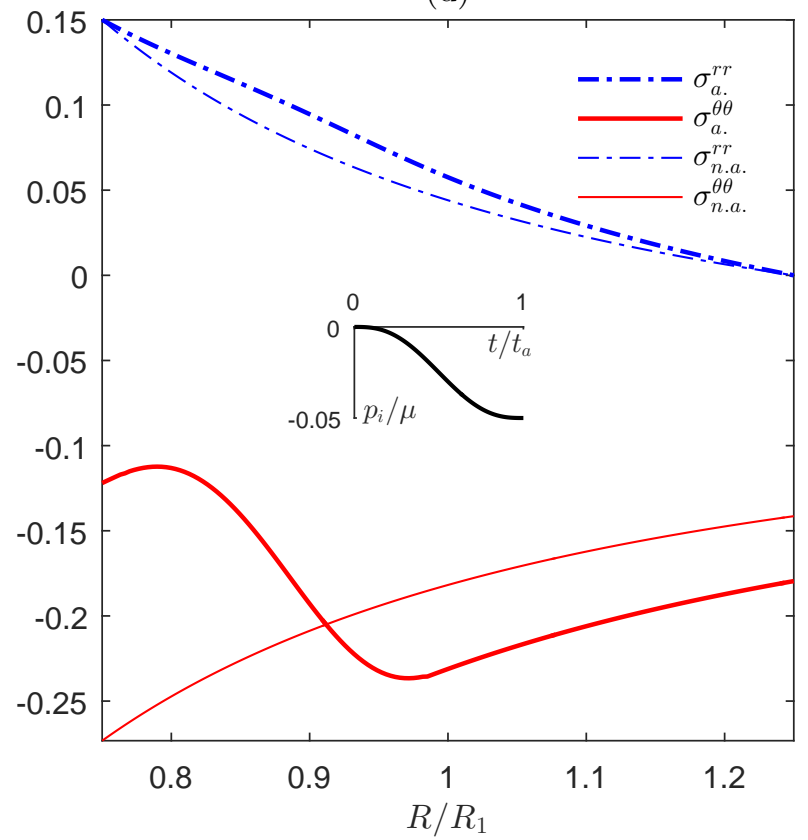

Figure 10: Comparison between an accreted and a non-accreted cylinder both with inner and outer radii $R_{a}$ and $R_{0}$, respectively, loaded by an internal pressure or suction $p_{e} / \mu=0.15$. The inner accreted cylinder was loaded during the accretion process by $p_{i}(t)$. (a) $p_{i}(t)>0$ and $p_{e}>0$, (b) $p_{i}(t)>0$ and $p_{e}<0$, (c) $p_{i}(t)<0$ and $p_{e}>0$, (d) $p_{i}(t)<0$ and $p_{e}<0$.

in this paper will be useful in solving such optimization problems.

\section{Acknowledgement}

This research was partially supported by AFOSR - Grant No. FA9550-12-1-0290, NSF - Grant No. CMMI 1130856, and ARO W911NF-16-1-0064. FS was supported by Sapienza University of Rome (Scholarship for 


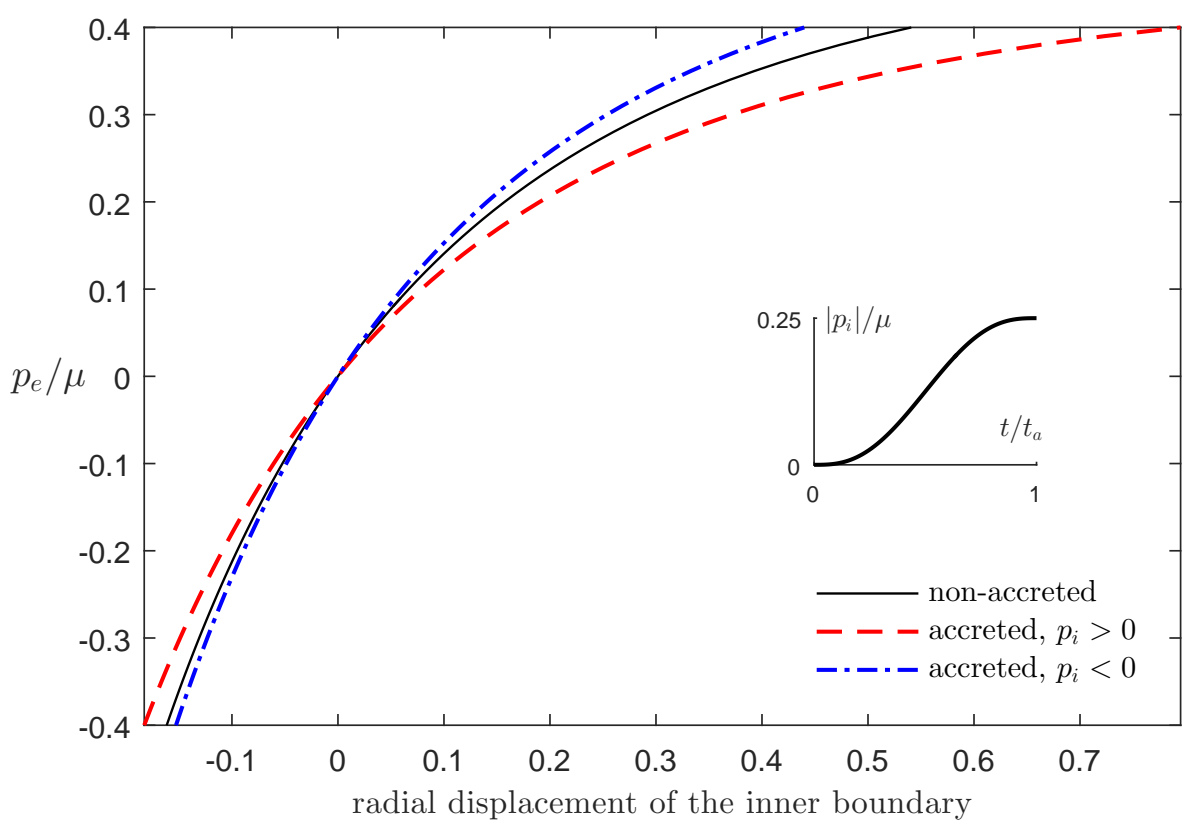

Figure 11: Loading of an inner-accreted cylinder $\left(R_{0} / R_{1}=1.25\right.$ and $\left.R_{a} / R_{1}=0.75\right)$ and its corresponding non-accreted cylinder. The relation between the pressure $p_{e} / \mu$ and the radial displacement of the inner boundary normalized with $R_{1}$ for accreted and non-accreted cylinders.

(a)

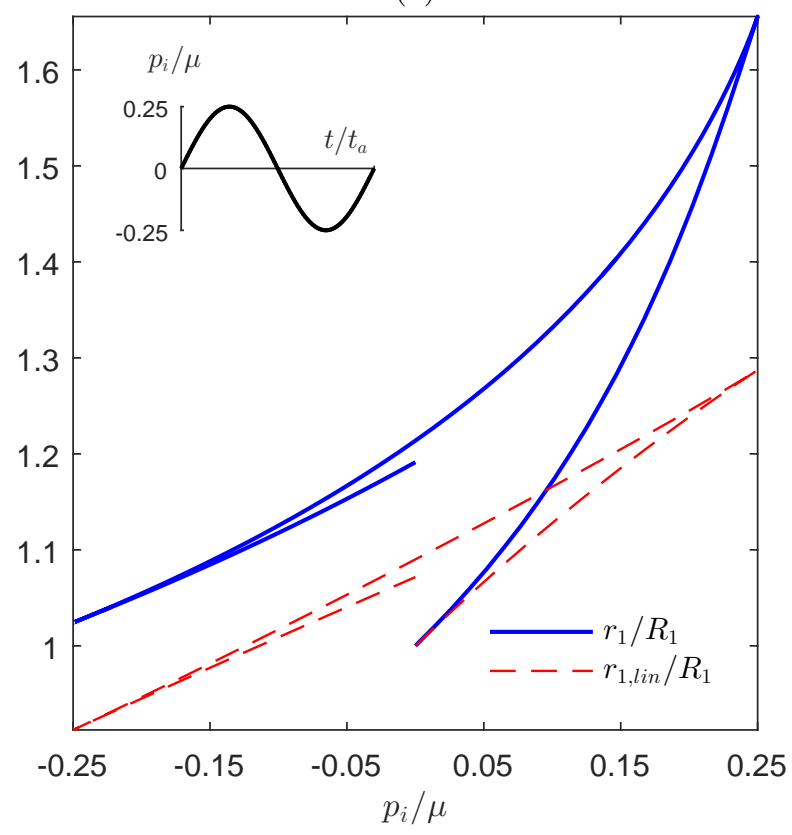

(b)

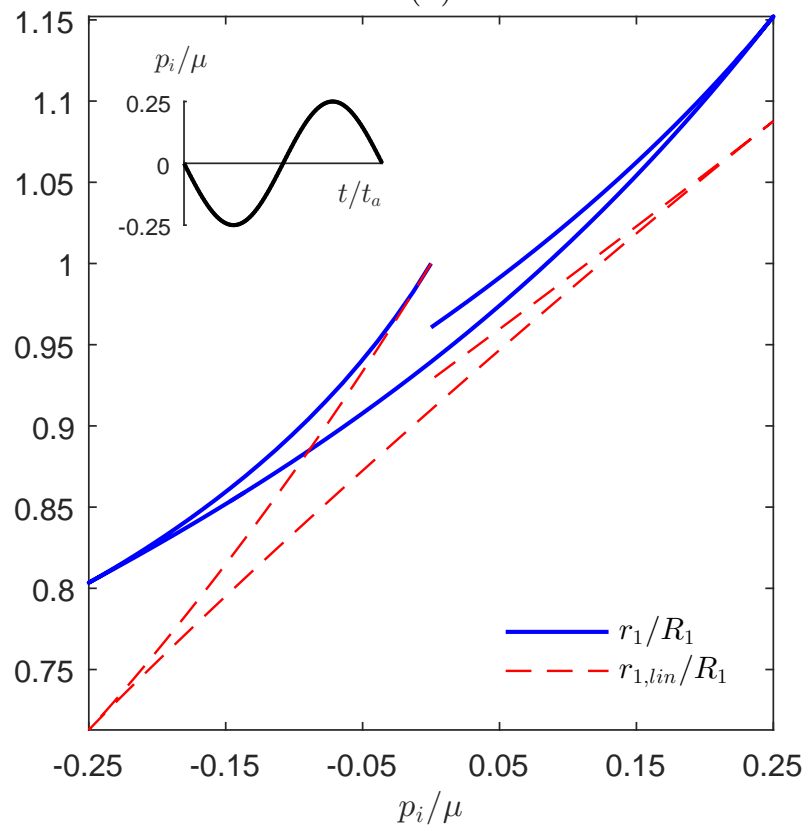

Figure 12: History-dependence (anelasticity) of the internal radius for (a) a compression-tension cycle and (b) a tensioncompression cycle during outer accretion of a cylindrical bar.

Specialization Abroad 2015). We are grateful to M. F. Shojaei for his help with the numerical simulations. 


\section{References}

H. C. Altmann. Formulas for computing stresses in center-wound rolls. Journal of the Technical Association of the Pulp and Paper Industry (TAPPI), 51:176-179, 1968.

S. S. Antman and M. M. Shvartsman. The shirink-fit problem for aeolotropic nonlinearly elastic bodies. Journal of elasticity, 37(2):157-166, 1994.

N. K. Arutyunyan, V. Naumov, and Y. N. Radaev. A mathematical model of a dynamically accreted deformable body. part 1: Kinematics and measure of deformation of the growing body. Izv. Akad. Nauk SSSR. Mekh. Tverd. Tela, (6):85-96, 1990.

M. Ben Amar and A. Goriely. Growth and instability in elastic tissues. Journal of the Mechanics and Physics of Solids, 53:2284-2319, 2005.

B. A. Bilby, B. R., and S. E. Continuous distributions of dislocations: a new application of the methods of non-riemannian geometry. Proceedings of the Royal Society of London A, 231(1185):263-273, 1955.

C. Brown and L. Goodman. Gravitational stresses in accreted bodies. In Proceedings of the Royal Society of London A: Mathematical, Physical and Engineering Sciences, volume 276, pages 571-576. The Royal Society, 1963.

C. B. Brown, R. J. Evans, and E. R. LaChapelle. Slab avalanching and the state of stress in fallen snow. Journal of Geophysical Research, 77:4570-4580, 1972.

A. D. Drozdov. Continuous accretion of a composite cylinder. Acta Mechanica, 128(1), 1998a.

A. D. Drozdov. Viscoelastic Structures: Mechanics of Growth and Aging. Academic Press, 1998b.

C. Eckart. The thermodynamics of irreversible processes. 4. the theory of elasticity and anelasticity. Physical Review, 73(4):373-382, 1948.

M. Epstein and G. A. Maugin. Thermomechanics of volumetric growth in uniform bodies. International Journal of Plasticity, 16:951-978, 2000.

K. Garikipati, E. M. Arruda, K. Grosh, H. Narayanan, and S. Calve. A continuum treatment of growth in biological tissue: the coupling of mass transport and mechanics. Journal of the Mechanics and Physics of Solids, 52(7):1595-1625, 2004.

A. Golgoon, S. Sadik, and A. Yavari. Circumferentially-symmetric finite eigenstrains in incompressible isotropic nonlinear elastic wedges. International Journal of Non-Linear Mechanics, 84:116-129, 2016.

A. E. Green, R. S. Rivlin, and R. T. Shield. General theory of small elastic deformations superposed on finite elastic deformations. Proceedings of the Royal Society A, 211(1104):128-154, 1952.

N. Hodge and P. Papadopoulos. A continuum theory of surface growth. In Proceedings of the Royal Society of London A: Mathematical, Physical and Engineering Sciences, volume 466, pages 3135-3152. The Royal Society, 2010.

J. Kadish, J. Barber, and P. Washabaugh. Stresses in rotating spheres grown by accretion. International Journal of Solids and Structures, 42(20):5322-5334, 2005.

A. Klarbring, T. Olsson, and J. Stalhand. Theory of residual stresses with application to an arterial geometry. Archives of Mechanics, 59(4-5):341-364, 2007.

K. Kondo. Geometry of elastic deformation and incompatibility. In K. Kondo, editor, Memoirs of the Unifying Study of the Basic Problems in Engineering Science by Means of Geometry, volume 1, Division C, pages 5-17. Gakujutsu Bunken Fukyo-Kai, 1955a. 
K. Kondo. Non-riemannian geometry of imperfect crystals from a macroscopic viewpoint. In K. Kondo, editor, Memoirs of the Unifying Study of the Basic Problems in Engineering Science by Means of Geometry, volume 1, Division D-I, pages 6-17. Gakujutsu Bunken Fukyo-Kai, 1955b.

S. Lychev. Universal deformations of growing solids. Mechanics of Solids, 46(6):863-876, 2011.

S. Lychev and A. Manzhirov. The mathematical theory of growing bodies. finite deformations. Journal of Applied Mathematics and Mechanics, 77(4):421-432, 2013a.

S. Lychev and A. Manzhirov. Reference configurations of growing bodies. Mechanics of Solids, 48(5):553-560, $2013 \mathrm{~b}$.

A. Manzhirov. The general non-inertial initial-boundaryvalue problem for a viscoelastic ageing solid with piecewise-continuous accretion. Journal of Applied Mathematics and Mechanics, 59(5):805-816, 1995.

A. V. Manzhirov. Mechanics of growing solids: New track in mechanical engineering. In ASME 2014 International Mechanical Engineering Congress and Exposition, pages V009T12A039-V009T12A039. American Society of Mechanical Engineers, 2014.

J. Marsden and T. Hughes. Mathematical Foundations of Elasticity. Dover, 1983.

V. Metlov. On the accretion of inhomogeneous viscoelastic bodies under finite deformations. Journal of Applied Mathematics and Mechanics, 49(4):490-498, 1985.

V. E. Naumov. Mechanics of growing deformable solids: a review. Journal of Engineering Mechanics, 120(2): 207-220, 1994.

R. W. Ogden. Non-linear elastic deformations. Dover, 1984.

J. J. Ong and O. M. O'Reilly. On the equations of motion for rigid bodies with surface growth. International Journal of Engineering Science, 42(19):2159-2174, 2004.

A. Ozakin and A. Yavari. A geometric theory of thermal stresses. Journal of Mathematical Physics, 51:032902, 2010.

E. K. Rodriguez, A. Hoger, and A. D. McCulloch. Stress-dependent finite growth in soft elastic tissues. Journal of Biomechanics, 27(455-467), 1994.

S. Sadik and A. Yavari. On the origins of the idea of the multiplicative decomposition of the deformation gradient. Mathematics and Mechanics of Solids, DOI:10.1177/1081286515612280, 2016a.

S. Sadik and A. Yavari. Geometric nonlinear thermoelasticity and the time evolution of thermal stresses. Mathematics and Mechanics of Solids, DOI: 10.1177/1081286515599458, $2016 \mathrm{~b}$.

S. Sadik, A. Angoshtari, A. Goriely, and A. Yavari. A geometric theory of nonlinear morphoelastic shells. Journal of Nonlinear Science, DOI: 10.1007/s00332-016-9294-9, 2016.

K. Schwerdtfeger, M. Sato, and K.-H. Tacke. Stress formation in solidifying bodies. solidification in a round continuous casting mold. Metallurgical and Materials Transactions B, 29(5):1057-1068, 1998.

J. Simo and J. Marsden. Stress tensors, riemannian metrics and the alternative descriptions in elasticity. In Trends and Applications of Pure Mathematics to Mechanics, pages 369-383. Springer, 1984.

R. Skalak, D. Farrow, and A. Hoger. Kinematics of surface growth. Journal of Mathematical Biology, 35(8): 869-907, 1997.

R. Southwell. Introduction to the Theory of Elasticity for Engineers and Physicists. Oxford University Press, 1941.

K. Takamizawa. Stress-free configuration of a thick-walled cylindrical model of the artery - an application of riemann geometry to the biomechanics of soft tissues. Journal of Applied Mechanics, 58:840-842, 1991. 
K. Takamizawa and T. Matsuda. Kinematics for bodies undergoing residual stress and its applications to the left ventricle. Journal of Applied Mechanics, 57:321-329, 1990.

H. P. Yagoda. Resolution of a core problem in wound rolls. Journal of Applied Mechanics, 47(4):847-854, 1980.

A. Yavari. A geometric theory of growth mechanics. Journal of Nonlinear Science, 20(6):781-830, 2010.

A. Yavari and A. Goriely. Riemann-Cartan geometry of nonlinear dislocation mechanics. Archive for Rational Mechanics and Analysis, 205(1):59-118, 2012a.

A. Yavari and A. Goriely. Weyl geometry and the nonlinear mechanics of distributed point defects. Proceedings of the Royal Society A, 468:3902-3922, 2012b.

A. Yavari and A. Goriely. Riemann-Cartan geometry of nonlinear disclination mechanics. Mathematics and Mechanics of Solids, 18(1):91-102, 2013a.

A. Yavari and A. Goriely. Nonlinear elastic inclusions in isotropic solids. Proceedings of the Royal Society A, 469:20130415, 2013b.

A. Yavari and A. Goriely. The geometry of discombinations and its applications to semi-inverse problems in anelasticity. Proceedings of the Royal Society A, 470:20140403, 2014.

A. Yavari and A. Goriely. The twist-fit problem: Finite torsional and shear eigenstrains in nonlinear elastic solids. Proceedings of the Royal Society A, 471:20150596, 2015.

A. Yavari and A. Ozakin. Covariance in linearized elasticity. ZAMP, 59(6):1081-1110, 2008. 